\title{
Correlation Models for Waste Tank Sludges and Slurries
}

\author{
L. A. Mahoney \\ D. S. Trent
}

July 1995

Prepared for

the U.S. Department of Energy

under Contract DE-AC06-76RLO 1830

Pacific Northwest Laboratory

Richland, WA 99352 


\section{DISCLAIMER}

Portions of this document may be illegible in electronic image products. Images are produced from the best available original document. 


\section{DISCLAIMER}

This report was prepared as an account of work sponsored by an agency of the United States Government. Neither the United States Government nor any agency thereof, nor any of their employees, make any warranty, express or implied, or assumes any legal liability or responsibility for the accuracy, completeness, or usefulness of any information, apparatus, product, or process disclosed, or represents that its use would not infringe privately owned rights. Reference herein to any specific commercial product, process, or service by trade name, trademark, manufacturer, or otherwise does not necessarily constitute or imply its endorsement, recommendation, or favoring by the United States Government or any agency thereof. The views and opinions of authors expressed herein do not necessarily state or reflect those of the United States Government or any agency thereof. 


\section{Summary}

This report presents the results of work conducted to support the TEMPEST computer modeling under the Flammable Gas Program (FGP) and to further the comprehension of the physical processes occurring in the Hanford waste tanks. The end products of this task are correlation models (sets of algorithms) that can be added to the TEMPEST computer code (Trent and Eyler 1993; Eyler et al. 1993) to improve the reliability of its simulation of the physical processes that occur in Hanford tanks. The correlation models can be used to augment, not only the TEMPEST code, but other computer codes that can simulate sludge motion and flammable gas retention.

This report presents the correlation models, also termed submodels, that have been developed to date. The submodel-development process is an ongoing effort designed to increase our understanding of sludge behavior and improve our ability to realistically simulate the sludge fluid characteristics that have an impact on safety analysis. The effort has employed both literature searches and data correlation to provide an encyclopedia of tank waste properties in forms that are relatively easy to use in modeling waste behavior. These properties submodels will be used in other tasks to simulate waste behavior in the tanks.

Density, viscosity, yield strength, surface tension, heat capacity, thermal conductivity, salt solubility, and ammonia and water vapor pressures were compiled for solutions and suspensions of sodium nitrate and other salts (where data were available), and the data were correlated by linear regression. In addition, data for simulated Hanford waste tank supernatant were correlated to provide density, solubility, surface tension, and vapor pressure submodels for multi-component solutions containing sodium hydroxide, sodium nitrate, sodium nitrite, and sodium aluminate. For many properties, the correlations fell within $10 \%$ of the data on which they were based. While the solution density and solids solubility can be modeled with true multi-component correlations, other properties must be estimated by treating the supernatant as a linearly weighted mixture of single-component aqueous solutions of sodium hydroxide, sodium nitrate, and sodium nitrite. Data have not been found to support the development of multi-component correlations for most waste properties.

A variety of rheological models were reviewed to find which best described the sludges in Hanford tanks. These models, including the power-law, Cross, Carreau, biviscous, Bingham plastic, Casson, Herschel-Bulkley, and Gay models, were compared with viscometric measurements for diluted and undiluted sludge from Tank 241-SY-101. The models were also compared with the stress/shear rate behavior of radioactive waste from storage tanks at Oak Ridge National Laboratory (ORNL). In the case of the SY-101 sludge, for which only high-shear-rate data were available, the 
Bingham and biviscous models provide the best fit. For the ORNL waste, with measurements taken at lower solids concentration and shear rates, the biviscous, Cross, and Carreau models provide the best data fits.

A multi-phase thermal conductivity submodel was identified for the combination of liquid, solid particles, and bubbles in Hanford tank sludge. The controlling parameters in the submodel are the volume fractions of the materials and the ratios of their thermal conductivities.

The governing equations for bubble rise (and, by analogy, for particle settling) are set forth in forms suitable for use in TEMPEST and other thermal-hydraulic codes. A submodel for the drag coefficients of single bubbles and swarms of bubbles was extracted from the literature and cast into a TEMPEST-compatible form. The submodel includes a modification to make it applicable to liquids in which particles are suspended, not only to pure liquids. A relationship for the virtual mass coefficients for single bubbles and swarms was also found. 


\section{Contents}



Contents $\ldots \ldots \ldots \ldots \ldots \ldots \ldots \ldots \ldots \ldots \ldots \ldots \ldots \ldots \ldots \ldots \ldots \ldots \ldots \ldots \ldots$

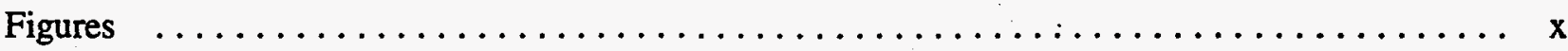

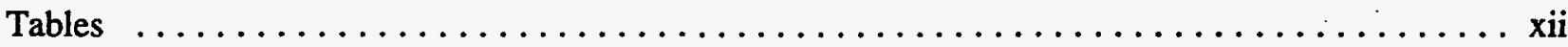

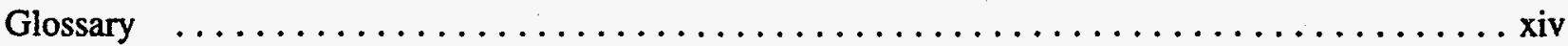

Acknowledgments $\ldots \ldots \ldots \ldots \ldots \ldots \ldots \ldots \ldots \ldots \ldots \ldots \ldots \ldots \ldots \ldots \ldots \ldots \ldots \ldots \ldots \ldots \ldots \ldots$

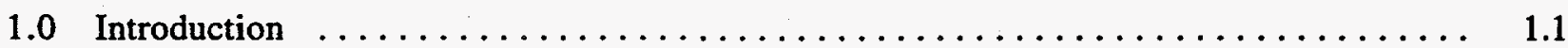

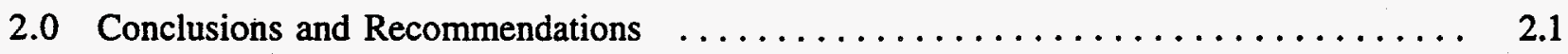

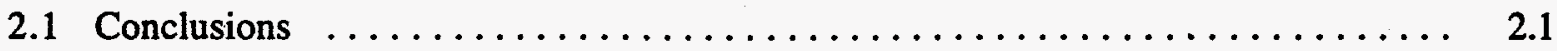

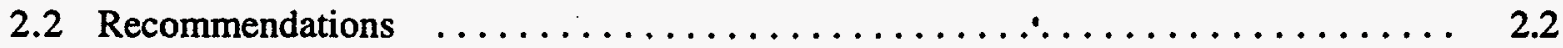

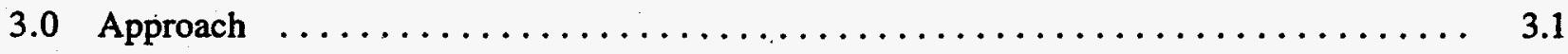

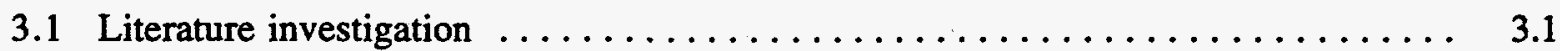

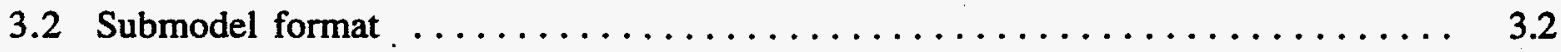

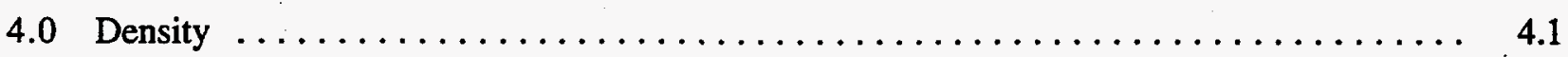



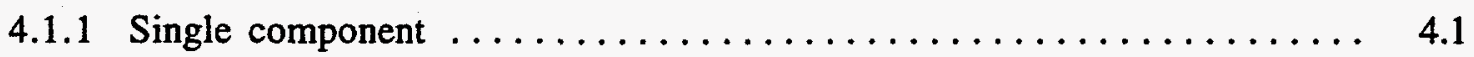

4.1.1.1 Template for $\rho_{d}$, the gas density $\left(\mathrm{kg} / \mathrm{m}^{3}\right) \ldots \ldots \ldots \ldots \ldots . \ldots . . \ldots$



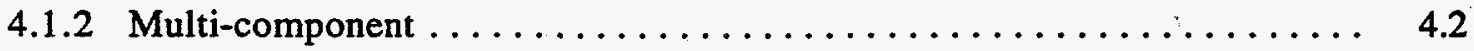

4.1.2.1 Template for $\rho_{d}$, the gas density $\left(\mathrm{kg} / \mathrm{m}^{3}\right) \ldots \ldots \ldots \ldots \ldots \ldots . \ldots .2$

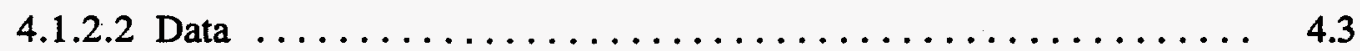

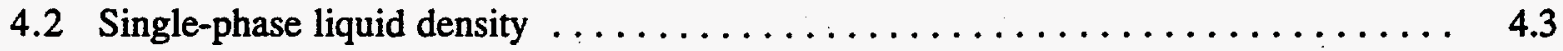

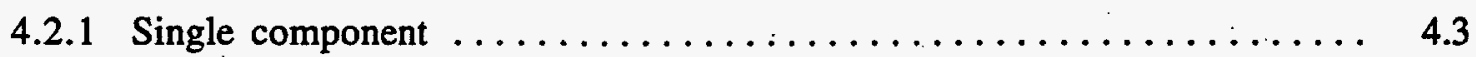

4.2.1.1 Template for $\rho_{c}$, the liquid density $\left(\mathrm{kg} / \mathrm{m}^{3}\right) \ldots \ldots \ldots \ldots . \ldots \ldots$

4.2.1.2 Data ........................... 4.3

4.2 .2 Multi-component $\ldots \ldots \ldots \ldots \ldots \ldots \ldots \ldots \ldots \ldots \ldots \ldots \ldots \ldots \ldots .4 .5$

4.2.2.1 Template for $\rho_{c}$, the liquid density $\left(\mathrm{kg} / \mathrm{m}^{3}\right) \ldots \ldots \ldots \ldots . \ldots .5$

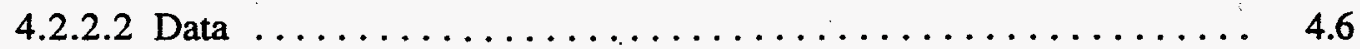






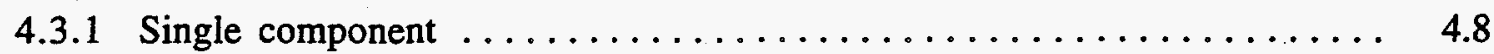

4.3.1.1 Template for $\rho_{\mathrm{s}}$, the solid density $\left(\mathrm{kg} / \mathrm{m}^{3}\right) \ldots \ldots \ldots \ldots \ldots \ldots \quad 4.8$

4.3.1.2 Data ............................... 4.8

4.3.2 Multi-component $\ldots \ldots \ldots \ldots \ldots \ldots \ldots \ldots \ldots \ldots \ldots \ldots \ldots \ldots, 4.9$

4.3.2.1 Template for $\rho_{s}$, the solid density $\left(\mathrm{kg} / \mathrm{m}^{3}\right) \ldots \ldots \ldots \ldots \ldots \ldots, 4.9$

4.3.2.2 Data ............................... 4.9

4.4 Liquid-solid (gasless) slurry density $\ldots \ldots \ldots \ldots \ldots \ldots \ldots \ldots \ldots \ldots .4 .10$

4.4.1 Template for $\rho_{\mathrm{m}}$, the gasless slurry density $\left(\mathrm{kg} / \mathrm{m}^{3}\right) \ldots \ldots \ldots \ldots \ldots \ldots 4.10$

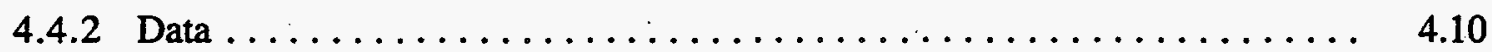

4.5 Liquid-solid-gas (bubbly) slurry density $\ldots \ldots \ldots \ldots \ldots \ldots \ldots \ldots \ldots \ldots, 4.11$

4.5.1 Template for $\rho_{b}$, the bubbly slurry density $\left(\mathrm{kg} / \mathrm{m}^{3}\right) \ldots \ldots \ldots \ldots \ldots, 4.12$

4.5.2 Data .................................. 4.12

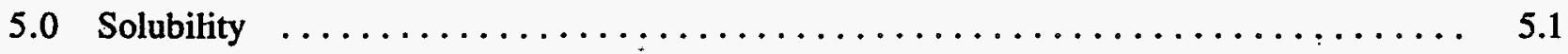

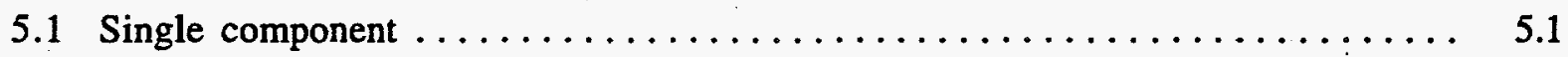

5.1.1 Template for $\mathrm{C}_{\mathrm{s}}$, the saturated concentration (wt \%) $\ldots \ldots \ldots \ldots \ldots \ldots .1$

5.1 .2 Data ....................................... 5.1

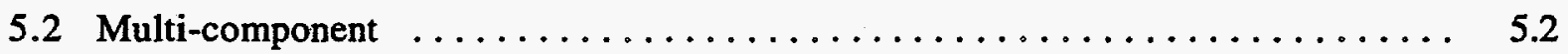

5.2.1 Template for $\mathrm{s}_{\mathrm{m}}$, the saturated concentration (molal) $\ldots \ldots \ldots \ldots \ldots .5 .3$





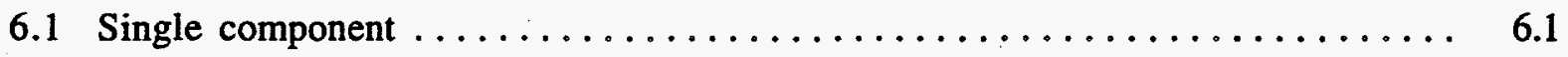

6.1.1 Template for $\mathrm{P}_{\mathrm{v}}$, the vapor pressure $(\mathrm{Pa}) \ldots \ldots \ldots \ldots \ldots \ldots \ldots \ldots, 6.1$

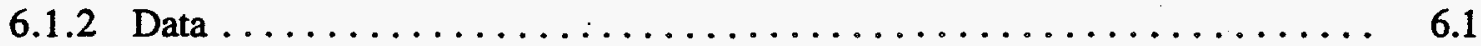

6.2 Multi-component $\ldots \ldots \ldots \ldots \ldots \ldots \ldots \ldots \ldots \ldots \ldots \ldots \ldots \ldots \ldots \ldots \ldots \ldots \ldots \ldots \ldots \ldots \ldots .2$

6.2.1 Template for $P_{\mathrm{v}}$, the vapor pressure $(\mathrm{Pa}) \ldots \ldots \ldots \ldots \ldots \ldots \ldots \ldots .6 \ldots \ldots$

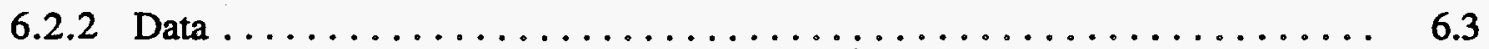




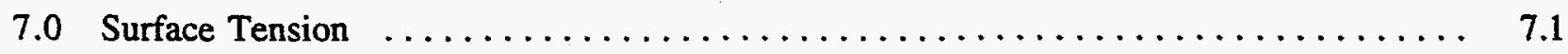

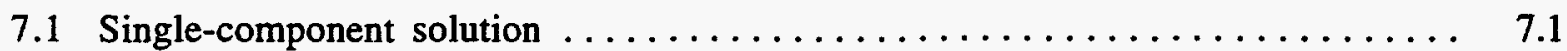

7.1.1 Template for $\sigma$, the surface tension $(\mathrm{N} / \mathrm{m}) \ldots \ldots \ldots \ldots \ldots \ldots \ldots \ldots .1$

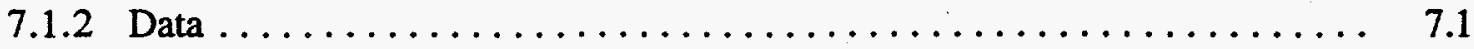

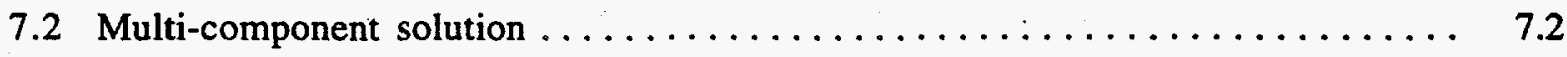

7.2.1 Template for $\sigma$, the surface tension $(\mathrm{N} / \mathrm{m}) \ldots \ldots \ldots \ldots \ldots \ldots \ldots \ldots \ldots \ldots \ldots .2$

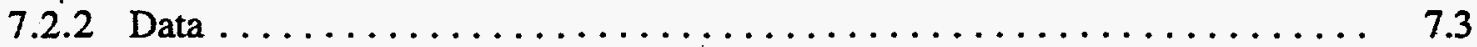



8.1 Liquid-solid (gasless) slurry yield strength $\ldots \ldots \ldots \ldots \ldots \ldots \ldots \ldots \ldots .6 .1$

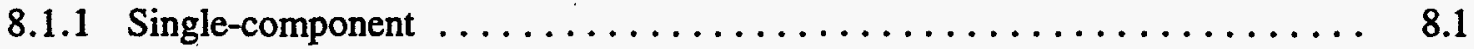

8.1.1.1 Template for $\tau_{\mathrm{s}}$, the gasless sludge yield strength $(\mathrm{Pa}) \ldots \ldots \ldots \ldots .2$

8.1 .1 .2 Data $\ldots \ldots \ldots \ldots \ldots \ldots \ldots \ldots \ldots \ldots \ldots \ldots \ldots \ldots \ldots \ldots \ldots .2$



8.2 Liquid-solid-gas (bubbly) slurry yield strength $\ldots \ldots \ldots \ldots \ldots \ldots \ldots \ldots \ldots, 8.3$



8.2.1.1 Templates for $\tau_{\mathrm{sb}}$, the bubbly sludge yield strength $(\mathrm{Pa}) \ldots \ldots \ldots \quad 8.4$

8.2.1.2 Data .............................. 8.5







9.2 Single-phase liquid rheological behavior $\ldots \ldots \ldots \ldots \ldots \ldots \ldots \ldots \ldots . \ldots .3$

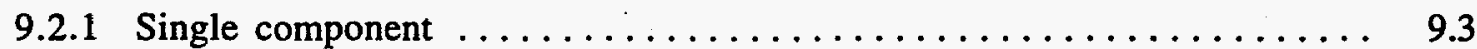

9.2.1.1 Template for $\mu_{c}$, the liquid viscosity (Pa-s) $\ldots \ldots \ldots \ldots \ldots \ldots .9 .4$

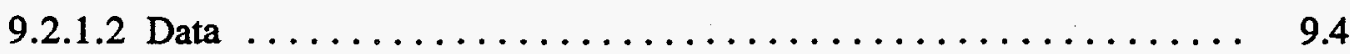

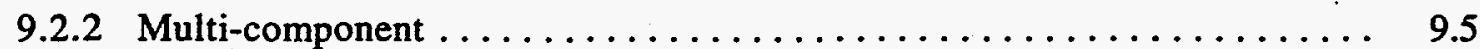

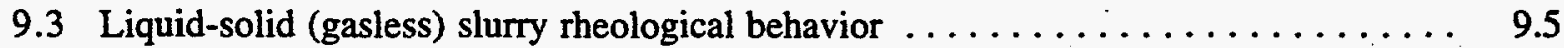

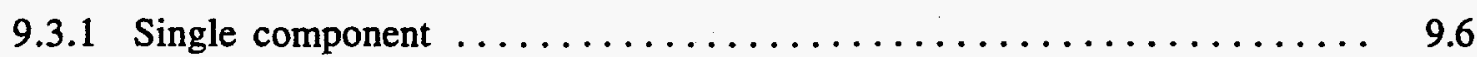

9.3.1.1 Templates for shear rate dependence of $\tau$, the fluid shear

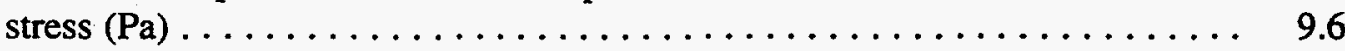

9.3.1.2 Templates for solids concentration dependence of $\tau$, the fluid

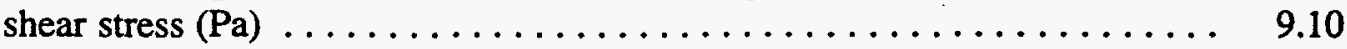


9.3.1.3 Data . . ........................... 9.11

9.3.1.3.1 Rheological submodels for the SY-101 sludge ..... 9.13

9.3.1.3.2 Rheological submodels for the ORNL W-28 sludge $\ldots 9.99$

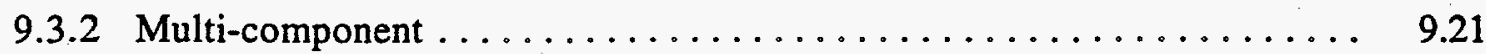



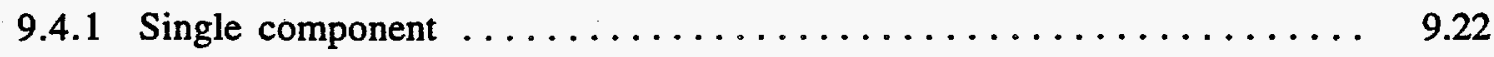

9.4.1.1 Templates for $\eta_{b}$, the bubbly slurry viscosity (Pa-s), and

$\tau_{0 b}$, the bubbly slurry yield stress $(\mathrm{Pa}) \ldots \ldots \ldots \ldots \ldots \ldots \ldots \ldots, 9.22$

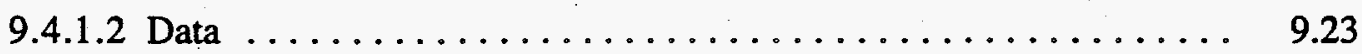

9.4.2 Multi-component $\ldots \ldots \ldots \ldots \ldots \ldots \ldots \ldots \ldots \ldots \ldots \ldots \ldots . \ldots \ldots .23$

9.5 Summary of recent relevant experimental literature $\ldots \ldots \ldots \ldots \ldots \ldots \ldots .9 .23$

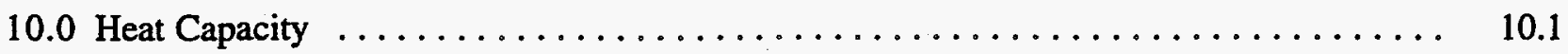

10.1 Single-phase gas heat capacity $\ldots \ldots \ldots \ldots \ldots \ldots \ldots \ldots \ldots \ldots \ldots \ldots . \ldots \ldots \ldots \ldots$



10.1.1.1 Template for $\mathrm{c}_{\mathrm{p}}$, the heat capacity of a gaseous species $(\mathrm{J} / \mathrm{kg} \mathrm{K}) \cdot 10.1$

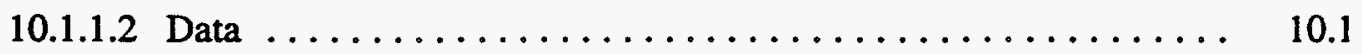

10.1.2 Multi-component $\ldots \ldots \ldots \ldots \ldots \ldots \ldots \ldots \ldots \ldots \ldots \ldots \ldots, 10.2$

10.1.2.1 Template for $\mathrm{c}_{\mathrm{pd}}$, the gas heat capacity $(\mathrm{J} / \mathrm{kg} \mathrm{K}) \ldots \ldots \ldots \ldots \quad 10.2$

10.1.2.2 Data ............................. 10.2

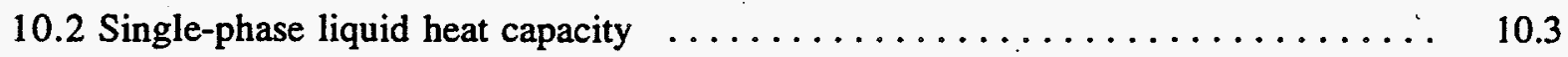

10.2.1 Single component $\ldots \ldots \ldots \ldots \ldots \ldots \ldots \ldots \ldots \ldots \ldots \ldots, 10.3$

10.2.1.1 Template for $c_{\mathrm{pc}}$, the liquid heat capacity $(\mathrm{J} / \mathrm{kg} \mathrm{K}) \ldots \ldots \ldots \ldots$

10.2.1.2 Data ............................... 10.3

10.2.2 Multi-component ............................ 10.4

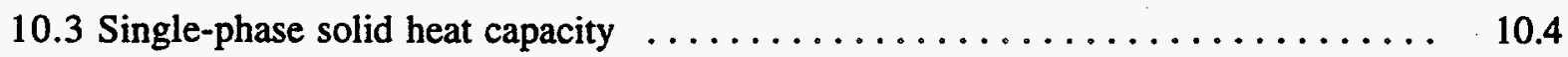



10.3.1.1 Template for $\mathrm{c}_{\mathrm{ps}}$, the solid heat capacity $(\mathrm{J} / \mathrm{kg} \mathrm{K}) \ldots \ldots \ldots \ldots \quad 10.5$

10.3.1.2 Data .................................. 10.5

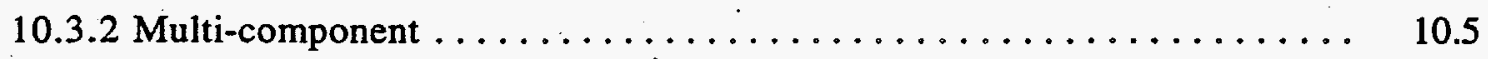

10.3.2.1 Template for $\mathrm{c}_{\mathrm{ps}}$, the solid density $(\mathrm{J} / \mathrm{kg} \mathrm{K}) \ldots \ldots \ldots \ldots \ldots \quad 10.6$

10.3.2.2 Data .............................. 10.6

10.4 Liquid-solid (gasless) slurry heat capacity $\ldots \ldots \ldots \ldots \ldots \ldots \ldots \ldots \ldots .10 .6$

10.4.1 Template for $c_{\mathrm{pm}}$, the gasless slurry heat capacity $(\mathrm{J} / \mathrm{kg} \mathrm{K}) \ldots \ldots \ldots \ldots 10.6$

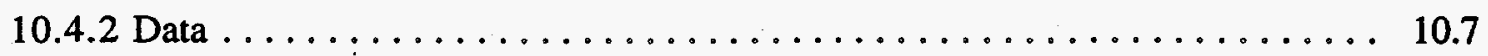


10.5 Liquid-solid-gas (bubbly) slurry heat capacity $\ldots \ldots \ldots \ldots \ldots \ldots \ldots \ldots, 10.7$

10.5.1 Template for $\mathrm{c}_{\mathrm{pb}}$, the bubbly slurry heat capacity $(\mathrm{J} / \mathrm{kg} \mathrm{K}) \ldots \ldots \ldots \ldots 10.7$

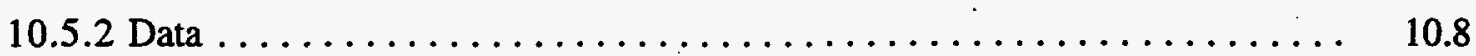

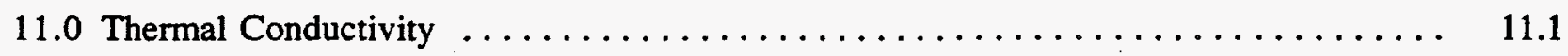

11.1 Single-phase gas thermal conductivity $\ldots \ldots \ldots \ldots \ldots \ldots \ldots \ldots \ldots \ldots, 11.1$

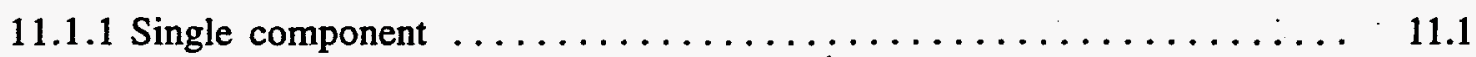

11.1.1.1 Template for $\mathrm{k}_{\mathrm{i}}$, the thermal conductivity of a gaseous

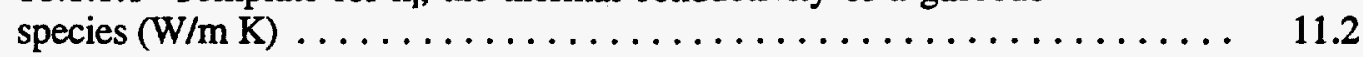

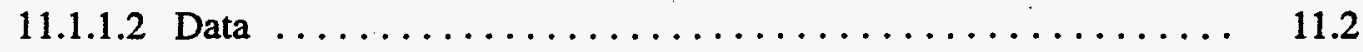

11.1.2 Multi-component $\ldots \ldots \ldots \ldots \ldots \ldots \ldots \ldots \ldots \ldots \ldots \ldots \ldots \ldots, 11.2$

11.1.2.1 Template for $k_{d}$, the gas thermal conductivity $(\mathrm{W} / \mathrm{m} \mathrm{K}) \ldots \ldots . .11 .3$

11.1.2.2 Data ............................... 11.3

11.2 Single-phase liquid thermal conductivity $\ldots \ldots \ldots \ldots \ldots \ldots \ldots \ldots \ldots . \ldots \ldots \ldots$

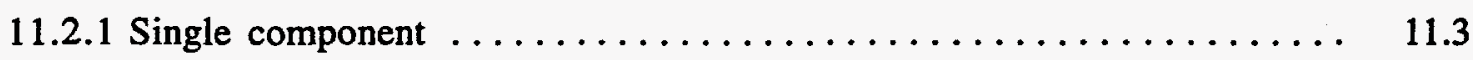

11.2.1.1 Template for $\mathrm{k}_{c}$, the liquid thermal conductivity $(\mathrm{W} / \mathrm{m} \mathrm{K}) \ldots \ldots \quad 11.4$

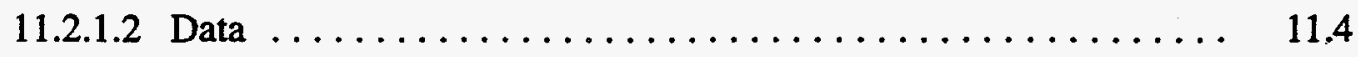

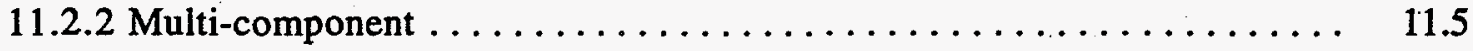

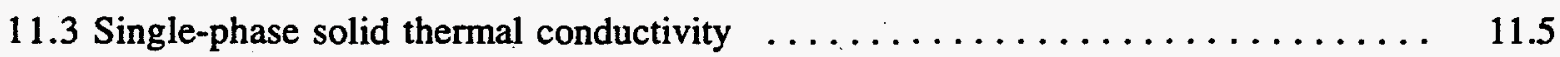

11.3.1. Single component $\ldots \ldots \ldots \ldots \ldots \ldots \ldots \ldots \ldots \ldots \ldots \ldots \ldots \ldots \ldots \ldots \ldots, 11.5$

11.3.1.1 Template for $\mathrm{k}_{\mathrm{s}}$, the solid thermal conductivity $(\mathrm{W} / \mathrm{m} \mathrm{K}) \ldots \ldots \quad 11.6$

11.3.1.2 Data ............................... 11.6

11.3.2 Multi-component $\ldots \ldots \ldots \ldots \ldots \ldots \ldots \ldots \ldots \ldots \ldots \ldots \ldots, 11.6$

11.4 Liquid-solid (gasless) slurry thermal conductivity $\ldots \ldots \ldots \ldots \ldots \ldots \ldots \ldots .11 .6$

11.4.1 Template for $\mathrm{k}_{\mathrm{m}}$, the gasless slurry thermal conductivity $(\mathrm{W} / \mathrm{m} \mathrm{K}) \ldots \ldots \quad 11.9$

11.4.2 Data ................................... 11.9

11.5 Liquid-solid-gas (bubbly) slurry thermal conductivity $\ldots \ldots \ldots \ldots \ldots \ldots \ldots \quad 11.10$

11.5.1 Template for $\mathrm{k}_{\mathrm{b}}$, the bubbly slurry thermal conductivity $(\mathrm{W} / \mathrm{m} \mathrm{K}) \ldots \ldots \quad 11.11$

11.5.2 Data ...................................... 11.11





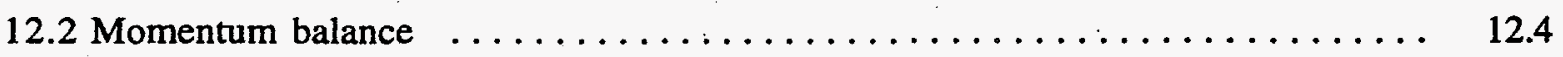

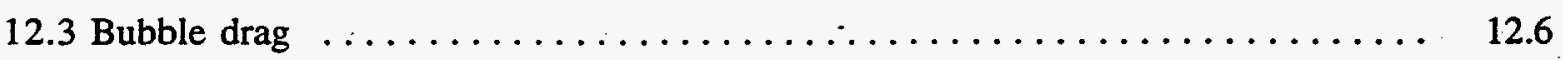

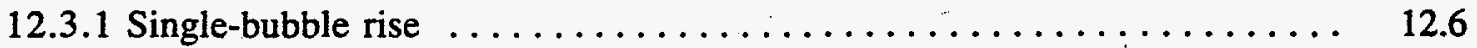

12.3.1.1 Fan and Tsuchiya submodel for single-bubble rise $\ldots \ldots \ldots \ldots . \quad 12.9$

12.3.1.2 Wallis submodel for single-bubble rise $\ldots \ldots \ldots \ldots \ldots \ldots \ldots, 12.9$ 


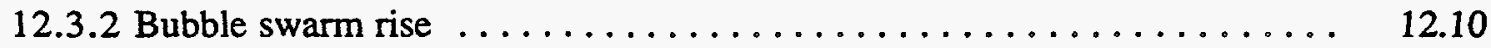

12.3.3 Rise in non-homogeneous or non-Newtonian Fluids $\ldots \ldots \ldots \ldots \ldots \ldots$

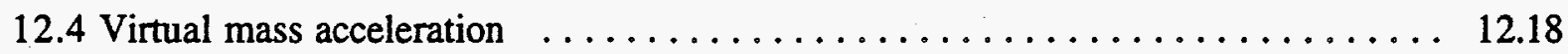

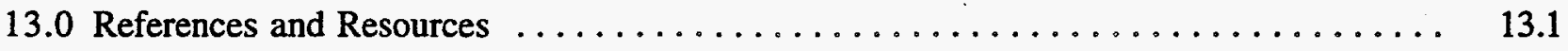



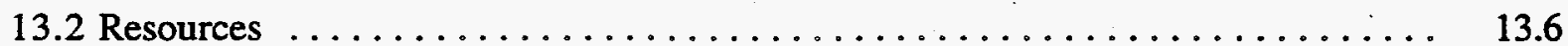




\section{Figures}

8.1 Tensile yield strength for a moist agglomerate of $71-\mu \mathrm{m}$ limestone particles $\ldots \ldots \ldots 8.10$

8.2 Tensile yield strength for a moist agglomerate of $185-\mu \mathrm{m}$ limestone particles $\ldots \ldots . \quad 8.10$

9.1 Rheological data and model fits (in the 0 to $50 / \mathrm{s}$ range) for SY-101 sludge

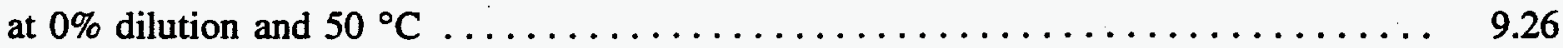

9.2 Rheological data and model fits (in the 0 to $50 / \mathrm{s}$ range) for SY-101 sludge at $10 \%$ dilution and $50^{\circ} \mathrm{C} \ldots \ldots \ldots \ldots \ldots \ldots \ldots \ldots \ldots \ldots \ldots \ldots \ldots \ldots \ldots \ldots \ldots \ldots . .27$

9.3 Rheological data and model fits (in the 0 to 50 /s range) for SY-101 sludge at $20 \%$ dilution and $50^{\circ} \mathrm{C} \ldots \ldots \ldots \ldots \ldots \ldots \ldots \ldots \ldots \ldots \ldots \ldots \ldots \ldots \ldots \ldots \ldots \ldots \ldots \ldots \ldots .28$

9.4 Rheological data and model fits (in the 0 to 50 /s range) for SY-101 sludge



9.5 Rheological data and model fits (in the 0 to $50 / \mathrm{s}$ range) for SY-101 sludge at $50 \%$ dilution and $50{ }^{\circ} \mathrm{C}$

9.6 Rheological data and model fits (in the 0 to $50 / \mathrm{s}$ range) for SY-101 sludge

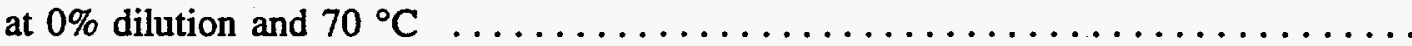

9.7 Rheological data and model fits (in the 0 to $50 / \mathrm{s}$ range) for SY-101 sludge

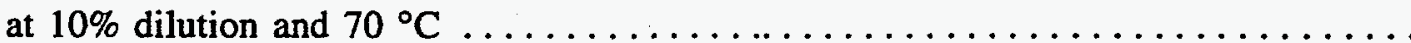

9.8 Rheological data and model fits (in the 0 to $50 / \mathrm{s}$ range) for $\mathrm{SY}-101$ sludge at $20 \%$ dilution and $70{ }^{\circ} \mathrm{C}$

9.9 Rheological data and model fits (in the 0 to $50 / \mathrm{s}$ range) for SY-101 sludge

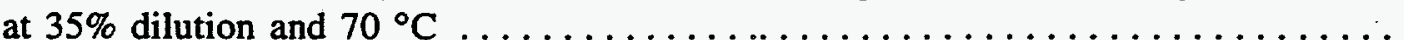

9.10 Rheological data and model fits (in the 0 to $50 / \mathrm{s}$ range) for SY-101 sludge

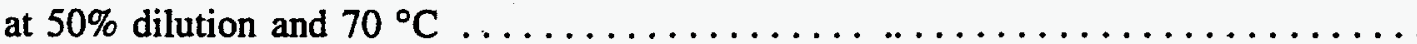

9.11 Rheological data and model fits (in the 0 to $50 / \mathrm{s}$ range) for SY-101 sludge at $0 \%$ dilution and $90^{\circ} \mathrm{C}$

9.12 Rheological data and model fits (in the 0 to $50 / \mathrm{s}$ range) for SY-101 sludge

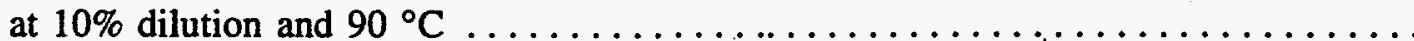

9.13 Rheological data and model fits (in the 0 to $50 / \mathrm{s}$ range) for SY-101 sludge at $20 \%$ dilution and $90^{\circ} \mathrm{C}$

9.14 Rheological data and model fits (in the 0 to $50 / \mathrm{s}$ range) for SY-101 sludge at $35 \%$ dilution and $90{ }^{\circ} \mathrm{C}$

9.15 Rheological data and model fits (in the 0 to $50 / \mathrm{s}$ range) for SY-101 sludge at $50 \%$ dilution and $90^{\circ} \mathrm{C}$ 
9.16 Tank $W-28$ data as fitted by a power-law model $\ldots \ldots \ldots \ldots \ldots \ldots . \ldots \ldots$

9.17 Tank $W-28$ data as fitted by the Cross model $\ldots \ldots \ldots \ldots \ldots \ldots \ldots \ldots \ldots \ldots . \ldots \ldots$

9.18 Tank $W-28$ data as fitted by the Carreau model . . . . . . . . . . . . . . . . 9.42



9.20 Tank W-28 data as fitted by the Herschel-Bulkley model $\ldots \ldots \ldots \ldots \ldots \ldots \ldots . .43$

9.21 Tank $W-28$ data as fitted by the Casson model $\ldots \ldots \ldots \ldots \ldots \ldots \ldots . \ldots \ldots$

9.22 Tank $W-28$ data as fitted by the Gay model $\ldots \ldots \ldots \ldots \ldots . \ldots \ldots . . \ldots \ldots$

12.1 Comparison of single-bubble rise submodels $\ldots \ldots \ldots \ldots \ldots \ldots \ldots \ldots \ldots \ldots \ldots \ldots$

12.2 Comparison of bubble swarm rise submodels $\ldots \ldots \ldots \ldots \ldots \ldots \ldots \ldots \ldots \ldots \ldots \ldots$ 


\section{Tables}

4.1 Density correlations $\left(\mathrm{kg} / \mathrm{m}^{3}\right)$ for aqueous solutions of several kindred sodium and

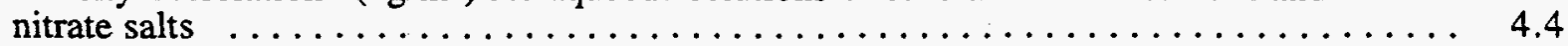

4.2 Density correlations $\left(\mathrm{kg} / \mathrm{m}^{3}\right)$ for multi-component aqueous solutions of tank waste

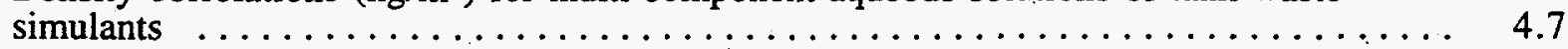

4.3 Densities of some solid salts present in the tank waste $\ldots \ldots \ldots \ldots \ldots \ldots \ldots \ldots \ldots$

4.4 Porosities estimated from Tank 241-SY-101 sludge and supernatant densities $\ldots \ldots \ldots 4.11$

5.1 Solubility relations $(w t \%)$ for aqueous solutions of some salts $\ldots \ldots \ldots \ldots \ldots .2$

5.2 Solubility relations $(w t \%)$ for the major salts in waste simulant solutions $\ldots \ldots \ldots \ldots$

6.1 Vapor pressure of water and ammonia $(\mathrm{Pa})$ in $\mathrm{NaOH}$ solution $\ldots \ldots \ldots \ldots \ldots . \ldots .2$

6.2 Vapor pressure of water and ammonia $(\mathrm{Pa})$ for two $S Y-101$ simulants $\ldots \ldots \ldots \ldots .3$

7.1 Surface tension correlations $(\mathrm{N} / \mathrm{m})$ for aqueous solutions of some sodium salts $\ldots \ldots 7.2$

7.2 Surface tension correlations $(\mathrm{N} / \mathrm{m})$ for two $\mathrm{SY}-101$ simulants $\ldots \ldots \ldots \ldots \ldots \ldots$

8.1 Static yield strength of $\mathrm{SY}-101$ sludge $(\mathrm{Pa}) \ldots \ldots \ldots \ldots \ldots \ldots \ldots \ldots \ldots \ldots \ldots \ldots \ldots$

9.1 Solids fraction versus tank dilution ratio at $50^{\circ} \mathrm{C} \ldots \ldots \ldots \ldots \ldots \ldots \ldots \ldots$

9.2 Correlated yield stress $\tau_{0}$ for SY-101 sludge fitted with a Bingham model $\ldots \ldots \ldots .15$

9.3 Correlated viscosity $\eta_{\infty}$ for SY-101 sludge fitted with a Bingham model $\ldots \ldots \ldots . .15$

9.4 Correlated low-shear-rate viscosity $\eta_{0}$ for SY-101 sludge fitted with a biviscous model . . . . . . . . . . . . . . . . 9.16

9.5 Correlated high-shear-rate viscosity $\eta_{\infty}$ for $S Y-101$ sludge fitted with a biviscous model $\ldots \ldots \ldots \ldots \ldots \ldots \ldots \ldots \ldots \ldots \ldots \ldots \ldots \ldots \ldots \ldots \ldots$

9.6 Correlated biviscosity constant B for SY-101 sludge fitted with a biviscous model $\ldots \ldots 9.16$

$9.7^{\circ}$ Correlation parameters for theological models fit to the ORNL MVST W-28 stress $/$ strain data . . . . . . . . . . . . . . . . . . . . . . . . . . . . . . 9.19

10.1 Heat capacity correlations $(\mathrm{J} / \mathrm{kg} \mathrm{K}$ ) for gases present in the waste gas $\ldots \ldots \ldots \ldots .2$

10.2 Heat capacity correlations $(\mathrm{J} / \mathrm{kg} \mathrm{K}$ ) for single-component aqueous solutions $\ldots \ldots \ldots 10.4$

10.3 Heat capacity correlations $(\mathrm{J} / \mathrm{kg} \mathrm{K})$ for some single-component salts $\ldots \ldots \ldots \ldots \ldots$

11.1 Thermal conductivity correlations $(\mathrm{W} / \mathrm{m} \mathrm{K})$ for gases present in the waste tank gas $\ldots \quad 11.2$ 11.2 Thermal conductivity correlations $(\mathrm{W} / \mathrm{m} \mathrm{K})$ for sodium chloride solution and water $\ldots \ldots 11.4$ 


\section{Glossary}

\section{Roman symbols}

$a_{k} \quad$ coefficients in a correlation, where $k$ is the index of the term

$\mathbf{a}_{\mathrm{R}}$ relative acceleration vector of a bubble in liquid

$\mathbf{a}_{R j} \quad$ relative acceleration vector of a $\mathrm{j}$-bin bubble in liquid

$A_{d j} \quad$ cross-sectional area of a bubble/particle in the $\mathrm{j}$ bin

$B$ correlation constant (used for modeling non-Newtonian fluids)

b radius (in the long direction) of an oblate ellipsoid

$b_{i} \quad$ in most uses, coefficient of the $i$-th species in a correlation;

in Section 9, coefficients in a correlation, where $i$ is the index of the term (not a species)

$\mathrm{Bo}_{\mathrm{w}}$ bubble Bond number, accounting for only the weight force on the bubble

$\mathrm{Bo}_{\mathrm{y}}$ bubble Bond number, accounting for both weight and yield forces on the bubble

c in Section 12, a correlation constant for bubble rise

$c_{p} \quad$ heat capacity $(\mathrm{J} / \mathrm{kg} \mathrm{K})$

$c_{p i} \quad$ heat capacity of a single species $\mathrm{i}$ in a mixture $(\mathrm{J} / \mathrm{kg} \mathrm{K})$

$\mathrm{c}_{\mathrm{pb}} \quad$ heat capacity of a bubbly slurry or sludge $(\mathrm{J} / \mathrm{kg} \mathrm{K})$

$\mathrm{c}_{\mathrm{pc}} \quad$ heat capacity of a solution (J/kg K)

$c_{p d}$ heat capacity of a mixture of gases $(J / \mathrm{kg} \mathrm{K})$

$c_{\mathrm{pm}} \quad$ heat capacity of a gasless slurry or sludge (J/kg K)

$c_{\mathrm{ps}} \quad$ heat capacity of a solid (J/kg K)

C weight percent of a solute in a single-component solution, or total weight percent of solutes in a multi-component solution

$C_{1} \quad$ fitting parameter for capillary yield strength

$\mathrm{C}_{2} \quad$ fitting parameter for pendular yield strength

$\mathrm{C}_{\mathrm{d}}$ drag coefficient for a bubble or particle

$\mathrm{C}_{\mathrm{dj}} \quad$ drag coefficient between a bubble or particle in the $\mathrm{j}$ bin and the surrounding liquid

$C_{i}$ the weight percent of a species $i$ in a mixture

$\mathrm{C}_{\mathrm{r}}$ the volume fraction of solid divided by the maximum packing fraction

$\mathrm{C}_{\mathrm{s}} \quad$ saturated concentration of a solute (wt \%)

$\mathrm{C}_{\mathrm{vmj}} \quad$ virtual mass coefficient for a bubble in the $\mathrm{j}$ bin

$d_{m, i, j} \quad$ joint concentration solubility coefficient for the effect of species $i$ and $j$ concentrations (in molality units) on the solubility (in molality) of species $\mathrm{m}$ 
D shear rate tensor $\left(\mathrm{sec}^{-1}\right)$

$D_{d} \quad$ volume-equivalent diameter of a bubble

$D_{E}$ diameter corresponding to the distance at which the fractional strain around an expanded bubble equals the ductility $\mathrm{E}$

$\mathrm{D}_{\mathrm{p}} \quad$ flow-equivalent diameter of a pore (m)

$D_{s} \quad$ flow-equivalent diameter of a particle

E ductility, or the fractional strain at which a sludge fails

Eo Eotvos number of a bubble (defined in Equation 12.14)

f in Section 9, a characteristic function in a dynamic yield stress model in Section 11, a characteristic function in a two-phase thermal conductivity model

$f_{m} \quad$ characteristic function in thermal conductivity of gasless sludge

$F_{e b} \quad$ force a bubble must supply to expand in sludge

$\mathrm{F}_{\mathrm{vm}} \quad$ virtual mass force of liquid on a bubble

g acceleration of gravity $\left(\mathrm{m} / \mathrm{s}^{2}\right)$

h in Section 4, depth of a bubble below the sludge surface (m); in Section 12, radius (in the short direction) of an oblate ellipsoid

$k$ thermal conductivity of a mixture (W/m K)

$\mathrm{k}_{\mathrm{b}}$ thermal conductivity of a bubbly sludge $(\mathrm{W} / \mathrm{m} \mathrm{K})$

$k_{c}$ thermal conductivity of a solution (W/m K)

$k_{d} \quad$ thermal conductivity of a gas (W/m K)

$\mathrm{k}_{\mathrm{f}} \quad$ thermal conductivity of the less conductive part of a two-component mixture

$k_{h}$ thermal conductivity of the more conductive part of a two-component mixture

$k_{i}$ thermal conductivity of species $i$ in a mixture $(W / m K)$

$k_{m} \quad$ thermal conductivity of the gasless sludge $(\mathrm{W} / \mathrm{m} \mathrm{K})$

$\mathrm{k}_{0}$ dissolution rate constant for a solid species (molality/s)

$k_{1} \quad$ precipitation rate constant for a solid species ( $\mathrm{sec}^{-1}$ )

$\mathrm{K}$ in Section 9, a non-Newtonian fluid's consistency index (Pa-s).

$\mathrm{K}_{\mathrm{b}} \quad$ correlation constant in bubble-rise equations

$\mathrm{K}_{\mathrm{i}} \quad$ solubility equilibrium constant for species $\mathrm{i}$

$\mathrm{m}$ in Section 4, molality of a solute (gmol solute/ $\mathrm{kg}$ water); in Section 9, an adjustment to yield stress in Section 12, an exponent in the hindered-settling relationship

$\mathrm{m}_{\mathrm{i}} \quad$ molality of species $\mathrm{i}$

Mo Morton number of a liquid (defined in Equation 12.13b)

$\mathrm{Mo}_{\mathrm{m}} \quad$ Morton number of a multi-phase mixture (defined in Equations 12.13b and 12.23)

$\mathrm{n} \quad$ in Section 8, the exponent in the yield strength correlation in Section 9, the structural (or behavioral) index for a non-Newtonian fluid; in Section 12, a correlation exponent for bubble rise 
$\mathrm{N}$ number of species in a phase

$\mathrm{N}_{0} \quad$ number density of bubbles before gas generation $\left(/ \mathrm{m}^{3}\right)$

$\mathrm{N}_{\mathrm{d}} \quad$ number density of bubbles or particles

$\mathrm{N}_{\mathrm{dj}} \quad$ number density of bubbles or particles in the $\mathrm{j}$ bin

$N_{v} \quad$ number of bubbles in a sphere of diameter $D_{E}$

$P$ pressure $(\mathrm{Pa})$

$P_{L} \quad$ pressure at the top of the sludge (non-convective) layer $(\mathrm{Pa})$

$P_{v} \quad$ partial pressure for a species in solution $(\mathrm{Pa})$

$Q_{i} \quad$ activity product for species $\mathrm{i}$

$r_{n, j} \quad$ rate of mass transfer from the $n$ bin to the $j$ bin of a bubble or particle population

$\mathrm{r}^{*} \quad$ dimensionless bubble radius, defined by Equation (12.19)

$\mathrm{R} \quad$ in Section 12, total mass reaction rate entering the bubbles or particles; elsewhere, the ideal gas constant

$R_{j} \quad$ mass reaction rate entering one bin (j) of bubbles or particles

$R_{N} \quad$ number rate of generation of bubbles or particles (number/ volume time)

$R_{N j} \quad$ number rate of generation of bubbles or particles in the $j$ bin (number/ volume time)

$s_{m} \quad$ solubility of aqueous species $m$ (molal)

$S \quad$ saturation (fraction of non-solid volume occupied by liquid)

$\mathrm{S}_{\mathbf{2}} \quad$ saturation at which imbibition ends

$S_{b} \quad$ saturation at which peak pendular strength is found

$S_{c} \quad$ saturation at which peak capillary strength is found

t temperature $\left({ }^{\circ} \mathrm{C}\right)$

$\mathrm{T}$ temperature (K)

$u_{c}$ velocity of the continuous (liquid) phase

$\mathrm{u}_{\mathrm{d}} \quad$ velocity of the dispersed (gas or solid) phase

$u_{d j} \quad$ velocity of one bin (j) of a dispersed (gas or solid) phase

$u_{R j} \quad$ velocity of a bubble/particle in the $j$ bin relative to the surrounding liquid

$\mathrm{u}^{*} \quad$ dimensionless bubble rise velocity, defined in Equation (12.18)

$\mathrm{U}_{\mathrm{b}}$ bubble rise velocity $(\mathrm{m} / \mathrm{s})$

$\mathrm{V}$ can represent either bubble rise velocity or particle settling velocity

$\mathrm{V}_{\mathrm{s}}$ terminal velocity of a particle $(\mathrm{m} / \mathrm{s})$

$w_{d} \quad$ weight percent of total dissolved solids in a multi-component solution

W molecular weight $(\mathrm{kg} / \mathrm{gmol})$

$\mathrm{W}_{\mathrm{i}} \quad$ molecular weight of the $\mathrm{i}$-th gaseous species $(\mathrm{kg} / \mathrm{gmol})$

$x_{i} \quad$ mole fraction of the $i$-th gaseous species 


\section{Greek symbols}

$\alpha \quad$ in Section 8, a fitting parameter for capillary contribution to yield strength in Section 11, a characteristic function in a two-phase thermal conductivity model

$\alpha_{m} \quad$ characteristic function in thermal conductivity of gasless sludge

$\beta \quad$ concentration exponent in a partial-pressure correlation

$\beta_{\mathrm{m}, \mathrm{i}} \quad$ joint temperature-concentration solubility coefficient for the effect of temperature and the concentration of species $i$ (in molality units) on the solubility of species $\mathrm{m}$

$\Delta \mathrm{L} \quad$ elongation (strain)

$\Delta \mathrm{L}_{\max }$ maximum elongation (strain) at failure

$\varepsilon_{c} \quad$ volume fraction of a continuous (liquid) phase of a two-phase mixture

$\varepsilon_{d} \quad$ volume fraction of a dispersed (gas) phase of a two-phase mixture

$\varepsilon_{\mathrm{s}} \quad$ volume fraction of particles in a solid-liquid mixture

$\varepsilon_{\mathrm{dj}} \quad$ volume fraction of one bin (j) of a dispersed (gas or solid) phase

$\varepsilon_{\mathrm{d}, \mathrm{m}} \quad$ maximum (packed) volume fraction of bubbles in a gas-liquid mixture

$\varepsilon_{\mathrm{s}, \mathrm{m}} \quad$ maximum (packed) volume fraction of particles in a solid-liquid mixture

$\phi \quad$ volume fraction of the less thermally-conductive component of a two-phase mixture

$\eta \quad$ non-Newtonian dynamic viscosity of a fluid $(\mathrm{Pa}-\mathrm{s})$

$\eta_{b} \quad$ non-Newtonian viscosity of a bubbly suspension (Pa-s)

$\eta_{m} \quad$ non-Newtonian viscosity of a gasless suspension (Pa-s)

$\eta_{0} \quad$ low-shear-rate dynamic viscosity of a non-Newtonian fluid (Pa-s)

$\eta_{\infty} \quad$ high-shear-rate dynamic viscosity of a non-Newtonian fluid (Pa-s)

$\kappa$ ratio of thermal conductivities in a mixture: $k_{m} / k_{f}$, always greater than unity

$\lambda$ parameter characterizing the relative acceleration

$\mu \quad$ Newtonian dynamic viscosity of a liquid $(\mathrm{Pa}-\mathrm{s})$

$\mu_{c} \quad$ Newtonian dynamic viscosity of a liquid in a two-phase mixture (Pa-s)

$\mu_{\mathrm{m}} \quad$ Newtonian dynamic viscosity of a multi-phase mixture (Pa-s) 
$\Pi_{D} \quad$ second invariant of the shear rate tensor $\left(\mathrm{sec}^{-2}\right)$

$\Pi_{\tau} \quad$ second invariant of the shear stress tensor $\left(\mathrm{Pa}^{2}\right)$

$\rho$ density, usually of a solution $\left(\mathrm{kg} / \mathrm{m}^{3}\right)$

$\rho_{b} \quad$ density of a solid-liquid-gas mixture, or bubbly sludge $\left(\mathrm{kg} / \mathrm{m}^{3}\right)$

$\rho_{c}$ density of a continuous fluid phase (liquid) $\left(\mathrm{kg} / \mathrm{m}^{3}\right)$

$\rho_{d} \quad$ density of a dispersed phase (gas) $\left(\mathrm{kg} / \mathrm{m}^{3}\right)$

$\rho_{i}$ density of the $\mathrm{i}$-th species $\left(\mathrm{kg} / \mathrm{m}^{3}\right)$

$\rho_{\mathrm{m}} \quad$ bulk density of a solid-liquid mixture, or gasless sludge $\left(\mathrm{kg} / \mathrm{m}^{3}\right)$

$\rho_{s} \quad$ density of a solid salt $\left(\mathrm{kg} / \mathrm{m}^{3}\right)$

$\sigma \quad$ surface tension $(\mathrm{N} / \mathrm{m})$

$\tau \quad$ shear stress tensor $(\mathrm{Pa})$

$\tau \quad$ tensile stress $(\mathrm{Pa})$

$\tau_{0} \quad$ dynamic yield stress of a fluid, specifically a gasless suspension (Pa)

$\tau_{0 \mathrm{~b}} \quad$ dynamic yield stress of a bubbly suspension ( $\left.\mathrm{Pa}\right)$

$\tau_{\mathrm{s}} \quad$ static shear yield strength of the gasless sludge $(\mathrm{Pa})$

$\tau_{\mathrm{sb}} \quad$ static shear yield strength of the bubbly sludge (Pa)

$\tau_{\mathrm{t}} \quad$ tensile yield strength $(\mathrm{Pa})$

$\xi \quad$ used in Section 9 to denote any general rheological property

$\zeta \quad$ an ellipsoidal coordinate characterizing an oblate bubble 


\section{Acronyms}

DST double-shell tank

EDTA ethylenediaminetetraacetic acid

FGP Flammable Gas Program

GRE gas release event

HEDTA hydroxyethylethylenediamine triacetic acid

LLW low-level waste

MVST Melton Valley Storage Tanks

MW - molecular weight

NCAW neutralized current acid waste

ORNL Oak Ridge National Laboratory

SST single-shell tank

TOC total organic carbon 


\section{Acknowledgments}

The authors wish to acknowledge P.A. Gauglitz, H.C. Reid, Y. Onishi, and P.R. Bredt for their assistance, and to thank them for the discussions and guidance they have offered. 


\subsection{Introduction}

The episodic Gas Release Events (GRE) that have characterized the behavior of several Hanford waste tanks for the past several years result from the entrapment of gases generated in the settled solids, or sludge, layer of the tank. Over a period of time, the accumulated gases reduce the bulk density of the solids settled on the tank bottom, until the density of the settled layer becomes less than that of the overlying supernatant. The solids layer then rises or is fractured and the gases are released. Heat generation and rapid temperature fluctuations are also tank management safety concerns.

Many technical, operational, and safety questions about in-tank processes have been addressed using the TEMPEST computer code (Trent and Eyler 1993; Eyler et al. 1993). Computational methods of analysis may be the only way to approach many of these questions. Physical scale modeling, for example, does not appear feasible for evaluating the release of gas trapped in waste sludge under a large hydrostatic pressure gradient.

The use of more appropriate correlations and other submodels for material properties and processes in the tank would allow a less conservative approach to be used in predictions of gas release, sludge flow, and tank heating. (Here the term, submodels, is used to indicate data and formulae that are subsidiary to modeling overall waste behavior.) The purpose of this report is to improve many of those submodels and provide them in units and forms that are mutually consistent and easily adapted for use in thermal-hydraulic codes such as TEMPEST.

The submodels include bubble rise in single-phase fluids and particulate suspensions, properties of aqueous solutions of nitrates and nitrites, yield strength and rheology of suspensions and other non-Newtonian fluids, and thermal conductivity of fluid-solid and liquid-solid-gas mixtures. In general, data were not found to support multi-component submodels of properties and processes. Thus, most of the submodels in this document do not fully account for the effect of chemical composition on waste properties. This document can be updated as future research provides data for multi-component effects.

The results of this study are summarized in Section 2. Section 3 describes the methodology of the literature search and the encyclopedic format in which the properties and processes submodels are described.

Density, viscosity, surface tension, thermal properties, vapor pressure, salt solubility, and yield strength data were compiled and correlated (by linear regression) for sodium nitrate and (where 
available) other salts. These correlation models are more precise and specific to the salts that are present in Hanford waste tanks than the salt solution parameters that are presently implemented in the TEMPEST code, and should improve the property estimates for the components of the sludge. Those property correlations are in Sections 4 (density), 5 (solubility), 6 (vapor pressure of water and ammonia), 7 (surface tension), 8 (yield strength), 10 (heat capacity), and 11 (thermal conductivity).

A variety of rheological correlation models (including the power-law, Cross, Carreau, biviscous, Bingham plastic, Casson, Herschel-Bulkley, and Gay models) were reviewed to determine which were suitable for the sludges in Hanford tanks. As discussed in Section 9, the biviscous and Bingham submodels were found to best represent the available rheological data for the SY-101 sludge. The rheological data for a more dilute sludge from ORNL storage tanks was best represented by the biviscous, Cross, and Carreau submodels.

Section 11 describes a multi-phase thermal conductivity model that is more suitable for the combination of liquid, solid particles, and bubbles in Hanford tank sludge than the current linear weighting approach. Improved thermal conductivity estimates should lead to better prediction of temperature-driven variations in properties and flows.

In Section 12, the governing equations for bubble rise (and, by analogy, for particle settling) are set forth in a form suitable for use in computational modeling. A correlation model for the drag coefficients of single bubbles and swarms of bubbles has been put into a TEMPEST-compatible form to improve the code's ability to predict bubble motion over a range of gas volume fractions. The submodel includes a modification to make it applicable to liquids in which particles are suspended, in addition to pure liquids. A relationship for the virtual mass coefficients for single bubbles. and swarms was also found. All of these submodel extensions, which are described in Section 12, will allow improved simulation of the relative motion of the two phases over the full range of flow regimes present in the tanks. 


\subsection{Conclusions and Recommendations}

The interim goals of this correlation model development study were achieved, with further work planned, as noted in Section 2.2. The literature provided improved submodels for bubble rise, sludge properties, sludge rheology, and the thermal conductivity of the sludge. The new submodels were compiled into forms that are consistent with each other and can be easily adapted for computational analysis.

\subsection{Conclusions}

The density, viscosity, surface tension, thermal properties, vapor pressure, heat capacity, and salt solubility were correlated (by linear regression) and compiled for sodium nitrate and (where available) other salts, in single-component and multi-component solutions. These data should aid in modeling the supernatant solution.

- Errors in most of the property correlations were less than $10 \%$, and those correlations provide the properties' dependence on concentration of salt(s) (over the range of solubility) and on temperature $\left(0-100^{\circ} \mathrm{C}\right)$.

Data from Tank 241-SY-101 were correlated to provide a submodel of the static (solid) yield stress of the sludge (non-convective layer).

A variety of rheological submodels (including the power-law, Cross, Carreau, biviscous, Bingham plastic, Casson, Herschel-Bulkley, and Gay submodels) were reviewed to determine which best fit the available rheological data for the sludges in Hanford tanks, including Tank 241-SY-101.

- The properties of a sludge from the Melton Valley Storage Tanks (MVST) of ORNL were described and found to be best modeled by the biviscous, Cross, or Carreau submodels, which do not include a yield stress. However, this ORNL sludge contains only $22 \mathrm{wt} \%$ (15 vol\%) undissolved solids, a concentration at which yield stress is often absent. The sludge in Tank $241-S Y-101$ contains 32 vol\% undissolved solids. It was found to be well described by the Bingham submodel, which includes yield stress. The apparent presence of a yield stress in SY-101 sludge (though not in ORNL sludge) is consistent with its higher solids concentration.

- The rheological behavior of sludge from Tank SY-101 was also well characterized by a biviscous submodel, but not enough low-shear-rate data were available to determine whether the biviscous submodel provided a better fit than the Bingham submodel.

- Several pertinent literature references were summarized to allow quick review of some of the methods of measurement and types of rheological submodels that have been used to describe suspensions and sludges. 
An appropriate three-phase thermal conductivity submodel from the literature was found for the combination of liquid, solid particles, and bubbles in Hanford tank sludge.

A relative-velocity submodel of bubble rise (and, by analogy, of particle settling) was assembled, including both drag and virtual mass coefficients for ensembles of bubbles over a wide range of void fractions.

- The drag coefficient submodel for bubbles is expected to perform well in the viscous, distorted-particle, and spherical regimes, which should cover the range of bubble sizes found in the tanks. The void fraction range of applicability includes that expected in Hanford tank sludge.

- A mixture viscosity for particle-in-liquid suspensions was defined to allow calculation of bubble drag coefficients in suspensions (such as the diluted sludge), as well as in single-phase liquids.

- The predicted bubble virtual mass coefficients are expected to contain little error for low gas volume fractions, 20 vol\% or less. These low gas fractions are consistent with those believed to be present in Hanford tanks.

In general, it was found that the values of many tank sludge physical properties depended not only on the amount of gas present but on the morphology of the gas phase. Density, heat capacity, thermal conductivity, and yield strength could differ depending on whether bubbles were present in a spherical form or in a dendritic, pore-occupying form.

\subsection{Recommendations}

Several open items are noted as a result of this work:

- The correlations for most solution and suspension properties are based on single-component solutions; the exceptions are the supernatant density and solids solubility correlations, which came from experiments with sludge simulants. For other properties, such as viscosity, the literature does not suggest how to deal with multi-component effects on properties except by simple linear composition weighting. The properties submodels should be converted to multi-component correlations, as more data become available.

- A solubility study of the tank fluid would serve to identify the composition and temperature effects on solute solubility. In addition, estimates of the rates of precipitation and dissolution would improve the prediction of relatively slow processes, such as boehmite precipitation. 
- Data and correlations for the diffusivities of chemical species in multi-component solutions should be added.

- Data and correlations for the generation of gas in the supernatant and sludge are lacking. The temperature and solids dependence of radiolytic and thermal gas generation are crucial to obtaining the distribution of gas generation, and therefore of void fraction, in the waste.

- It has been observed that at low strain rate the SY-101 waste exhibits time-dependent (thixotropic) shear-thinning behavior. This indicates the microstructure of the sludge is undergoing a change of configuration. The degree of thixotropy will affect the fluid motion in parts of the tank where the strain rate is less than $150 \mathrm{sec}^{-1}$ (as reported by Tingey et al. 1994). In addition, qualitative evidence shows the yield stress decreases with the cumulative amount of shear the material has undergone. No attempt has been made to quantify these time-dependent or history-dependent effects.

- Fluids that exhibit a yield stress, as many suspensions do, may trap bubbles that are too small to exert a fluid stress greater than the yield stress. A method for calculating the maximum trapped bubble size would improve the bubble rise model in Section 12.

- Bubble coalescence and resulting changes in the bubble size distribution should be included in the bubble rise model, if data become available. 


\subsection{Approach}

The objective of this work was to survey the literature for several types of models needed for the tank studies, and to put those models into an encyclopedia of closed-form equation submodels that are consistent and compatible with computational modeling.

\subsection{Literature investigation}

The investigation procedure was to conduct a computerized literature search on a range of related topics, and pursue the most relevant subset of papers that were available through the Hanford Library. In many cases, further useful references were identified from the bibliographies of the computer-located set of papers, or from the recommendations of other members of the modeling team.

The literature search of the science and engineering literature abstracts covered four broad topics: solution and sludge thermodynamic properties (Sections 4, 5, 6, 7, and 10), sludge strength and rheology (Sections 8 and 9), sludge thermal conductivity (Section 11), and bubble rise (Section 12). The keywords that were used and the period of record covered by each search are included in the following paragraphs.

Sections 4-7, and 10 keywords: Sodium nitrate or nitrite, properties, aqueous solutions, density, viscosity, heat capacity, concentrated solutions. The entire period of record available in the Hanford Technical Library was reviewed.

Sections 8 and 9 keywords: Non-Newtonian liquids, rheology of slurries or sludges or muds or pastes, viscoplastic or pseudoplastic materials, Bingham, Carreau, Herschel-Bulkley, yield stress, power law, rheology of foams. Only the period of record from 1985 forward was reviewed. This search was augmented by reviewing the past two years of the Journal of Rheology.

Section 11 keywords: Thermal or electrical conductivity of mixtures or dispersions, multiphase, particles in continuum, discrete phase, continuous phase, composite electrolytes, percolation model. Only the period of record from 1985 forward was reviewed. Although thermal conductivity was the property to be used in tank modeling, electrical conductivity literature was also searched because electrical and thermal conduction are closely analogous processes.

Section 12 keywords: Bubbles, coalescence, breakup, wake capture, rise velocity, drag, terminal velocity, swarms, swarm dynamics, fluidized bed, suspension, particles, bubbly flow, slug 
flow. Only the period of record from 1985 forward was reviewed because of the large quantity of literature. The search was augmented by using the extensive bibliography in Stewart (1986).

The reference portion of Section 13 lists for each topic the references actually used to devise models for tank studies. The literature resources that were relevant, but not incorporated into models, are listed in the resources part of Section 13.

\subsection{Submodel format}

The submodels presented in this document fall into two categories: physical processes (Section 12, Bubble Rise) and physical properties (the other sections). Section 12 is written in the form of a description of submodels for bubble rise in solutions and suspensions and a discussion of their merits. The other sections, which contain submodels of physical properties of waste, are organized according to the physical phases and chemical components that are of interest.

Each physical properties section is divided into subsections for properties of the different phases (gas, liquid, and solid, as appropriate) and the multi-phase mixtures (gasless and bubbly suspensions). Each of these subsections is subdivided into single-component and multi-component models. Finally, each of these subsections is divided into a template section and a data section.

The template provides a general equation format to be used for all materials that have the property in question. That is, all the materials are correlated in terms of the same types of functional dependence on temperature, concentrations, and so on. The data section then provides the coefficients, exponents, and other factors to put into the template to obtain the property submodel for each material. The correctness of fit and the correlation error are also discussed in the data section. A data section may also contain some theoretical discussion or background material pertinent to the property. This encyclopedic arrangement should simplify any future additions of new properties or information and provide the clearest format for submodel definition. 


\subsection{Density}

Density is an essential property for waste tank modeling. The timing of gas release events (GREs) depends on the difference in density between the sludge and the supernatant. This density difference between gas and liquid is one of the controlling properties for bubble rise. In turn, the sludge density depends on the solid density and on whether bubbles are present in dendritic or spherical form. The variation of the fluid density is the driving force for natural convection, which determines the extent of mixing under non-pumped conditions; temperature causes part of the density variation.

This chapter begins by providing correlations and other submodels for the density of single phases, the gas in the bubbles (Section 4.1), the solution liquid (Section 4.2), and the solids (Section 4.3). Section 4.4 specifies a submodel for the gasless slurry density, and Section 4.5 discusses the bubbly slurry density.

\subsection{Single-phase gas density}

Gas is believed to be present in the sludge in the form of small dendritic or spherical bubbles among the sludge particles. The bubble gas density, therefore, has an effect on the sludge density, as well as on bubble rise velocities.

\subsubsection{Single component}

Because the gas in the tanks is at near-ambient temperatures and pressures, the ideal gas law can be used to estimate the gas density. The gas can be treated as a single-component phase, as in Equation (4.1), if an average molecular weight is used to represent the several gaseous species that are present.

\subsubsection{Template for $p_{d}$, the gas density $\left(\mathrm{kg} / \mathrm{m}^{3}\right)$}

$$
\rho_{d}=\frac{W_{d} P}{R T}
$$

Range: temperature greater than $250 \mathrm{~K}$; pressure less than $10 \mathrm{~atm}$ 


$$
\text { where } \begin{aligned}
\mathrm{W}_{\mathrm{d}} & =\text { mole-averaged molecular weight }(\mathrm{kg} / \mathrm{gmol}) \\
\mathrm{P} & =\text { pressure }(\mathrm{Pa}) \\
\mathrm{R} & =\text { ideal gas constant }=8.314 \mathrm{~Pa} \mathrm{~m} / \mathrm{gmol} \mathrm{K} \\
\mathrm{T} & =\text { temperature }(\mathrm{K})
\end{aligned}
$$

\subsubsection{Data}

The gas generated by the waste in Tank 241-SY-101 consists of hydrogen (molecular weight $0.002 \mathrm{~kg} / \mathrm{gmol}$ ), nitrogen (MW 0.028), nitrous oxide (MW 0.044), methane (MW 0.016), carbon monoxide (MW 0.030), water vapor (MW 0.018), and ammonia (MW 0.017). The composition of the gas varies with the local hydrostatic pressure; at $1.25 \mathrm{~atm}$ it has been estimated as $29.0 \mathrm{~mol}_{2} \mathrm{H}_{2}$, $24.6 \% \mathrm{~N}_{2} \mathrm{O}, 19.7 \% \mathrm{NH}_{3}, 21.7 \% \mathrm{~N}_{2}, 0.5 \% \mathrm{CH}_{4}, 0.5 \% \mathrm{CO}, 4.0 \% \mathrm{H}_{2} \mathrm{O}$ (Pasamehmetoglu 1995, Table 4.3.3.3-3). At this composition, $W_{d}=0.0218 \mathrm{~kg} / \mathrm{gmol}$. This value of $\mathrm{W}_{\mathrm{d}}$ is within about $2 \%$ of the values estimated for gas compositions at local pressures up to $2 \mathrm{~atm}$.

The gases in the waste can be described by the ideal gas assumption with an error of less than $1 \%$, for temperature greater than $250 \mathrm{~K}$ and pressure less than 10 atm (Perry and Chilton 1973).

\subsubsection{Multi-component}

Alternately, the gas can be represented in multi-component terms, as in Equation (4.2). Because of the linearity of the ideal gas law, the multi-component and single component equations are equivalent.

\subsubsection{Template for $\rho_{d}$, the gas density $\left(\mathrm{kg} / \mathrm{m}^{3}\right)$}

$$
\rho_{d}=\frac{P}{R T} \sum_{i=1}^{N} x_{i} W_{i}
$$

Range: temperature greater than $250 \mathrm{~K}$; pressure less than $10 \mathrm{~atm}$

$$
\text { where } \begin{aligned}
\mathrm{x}_{\mathrm{i}} & =\text { mole fraction of the } \mathrm{i}-\mathrm{th} \text { species of } \mathrm{N} \text { gaseous species } \\
\mathrm{W}_{\mathrm{i}} & =\text { molecular weight of the } \mathrm{i}-\mathrm{th} \text { gaseous species }(\mathrm{kg} / \mathrm{gmol}) \\
\mathrm{P} & =\text { pressure }(\mathrm{Pa}) \\
\mathrm{R} & =\text { ideal gas constant }=8.314 \mathrm{~Pa} \mathrm{~m} / \mathrm{gmol} \mathrm{K} \\
\mathrm{T} & =\text { temperature }(\mathrm{K})
\end{aligned}
$$




\subsubsection{Data}

The molecular weights and composition data for the gases in Tank 241-SY-101 wastes are the same as those given in Section 4.1.1.2.

\subsection{Single-phase liquid density}

The waste tank supernatant solution is primarily a combination of $\mathrm{NaNO}_{3}, \mathrm{NaNO}_{2}$, and other nitrate and sodium salts. The nitrate and nitrite salts account for most of the density characteristics of the supernatant. Sodium aluminate, sodium hydroxide, and other salts and organic compounds are present, but in much smaller quantities.

\subsubsection{Single component}

As a first approximation, the supernatant liquid in Tank 241-SY-101 and other Hanford waste tanks can be treated as a single-component solution, using one of the major aqueous species as a surrogate for the rest. A template for such an approach is given in Equation (4.3).

\subsubsection{Template for $\rho_{c}$, the liquid density $\left(\mathrm{kg} / \mathrm{m}^{3}\right)$}

$$
\rho_{c}=a_{0}+a_{1} C+a_{2} C^{3}+a_{3} T+a_{4} T+a_{5} C T+a_{6} T^{2}
$$

Range: 277-373 K; less than saturated concentration

$$
\text { where } \begin{aligned}
a_{k} & =\text { coefficients obtained by data correlation } \\
\mathbf{C} & =\text { concentration of solute in weight percent of the total solution }
\end{aligned}
$$

\subsubsection{Data}

The densities of aqueous solutions of several sodium salts that are present in tank waste are given in the form of correlations in Table 4.1. These data were correlated with a linear regression to provide the temperature and concentration dependence of the density. With the exception of $\mathrm{NaNO}_{2}$, all of the salt solution densities were correlated over a range of temperatures; for sodium nitrite, data were available only at the temperature of $15^{\circ} \mathrm{C}$. 
Some of the sodium salts have strongly similar effects on liquid density. At $15^{\circ} \mathrm{C}$, the correlations of $\mathrm{NaNO}_{3}$ and $\mathrm{NaNO}_{2}$ solution densities are within a few percent of each other. The data for $\mathrm{NaCl}$ solution density in Table 3-90 of Perry and Chilton (1973) show it as within $1.5 \%$ of the density of $\mathrm{NaNO}_{3}$ at the same weight percent concentration.

The solution density for $\mathrm{HNO}_{3}$ was correlated in the same manner to show that the template can match data even for $100 \%$ solute concentrations. This close fit provides some reassurance that the other salts' correlations will also predict well at high concentrations, even though the data for concentrations near saturation were lacking.

Table 4.1. Density correlations $\left(\mathrm{kg} / \mathrm{m}^{3}\right)$ for aqueous solutions of several kindred sodium and nitrate salts. The correlations all use the template in Equation (4.3).

\begin{tabular}{|c|c|c|c|c|c|c|}
\hline Salt & $a_{0}$ & $a_{1}$ & $a_{2} * 10^{3}$ & $\mathbf{a}_{3}$ & $a_{4}$ & $a_{5} * 10^{3}$ \\
\hline $\mathrm{NaNO}_{3}(1)$ & 1523.5 & 9.4989 & 0.6231 & -1.0598 & -63250 & -8.403 \\
\hline $\mathrm{NaCl}$ & 1584.1 & 9.0608 & 0.6679 & -1.1461 & -73500 & -6.127 \\
\hline $\mathrm{NaOH}$ & 1370.7 & 12.856 & -0.2438 & -0.8298 & -38090 & -5.666 \\
\hline $\mathrm{Na}_{2} \mathrm{CO}_{3}$ & 1665.4 & 13.231 & 0.8024 & -1.2710 & -86560 & -9.404 \\
\hline $\mathrm{HNO}_{3}$ & 1473.3 & 11.173 & -0.1461 & -0.9664 & -58530 & -15.36 \\
\hline $\mathrm{NaNO}_{3}{ }^{(2)}$ & 998.6 & 7.078 & 0.6231 & -- & -- & -- \\
\hline $\mathrm{NaNO}_{2}$ & 999.0 & 6.799 & 0.5716 & -- & -- & -- \\
\hline
\end{tabular}

(1). These data describe the $\mathrm{NaNO}_{3}$ correlation over the full temperature range.

(2) This correlation describes $\mathrm{NaNO}_{3}$ solution density only at $15^{\circ} \mathrm{C}$, to allow easy comparison with the $\mathrm{NaNO}_{2}$ data. 
Table 4.1. (continued)

\begin{tabular}{|c|c|c|c|c|}
\hline Salt & $\begin{array}{l}\text { Temp. } \\
\text { range } \\
(\mathrm{K})\end{array}$ & $\begin{array}{l}\text { Conc. } \\
\text { range } \\
\text { (wt \%) }\end{array}$ & $\begin{array}{c}\text { Corre- } \\
\text { lation } \\
r^{2}\end{array}$ & $\begin{array}{c}\text { Max. error } \\
\left(\mathrm{kg} / \mathrm{m}^{3}\right)\end{array}$ \\
\hline $\mathrm{NaNO}_{3}(1)$ & $277-373$ & $0-45$ & 0.9998 & 2.7 \\
\hline $\mathrm{NaCl}$ & $277-373$ & $0-26$ & 0.9998 & 2.1 \\
\hline $\mathrm{NaOH}$ & $277-373$ & $0-50$ & 0.9999 & 4.8 \\
\hline $\mathrm{Na}_{2} \mathrm{CO}_{3}$ & $277-373$ & $0-30$ & 0.9999 & 2.0 \\
\hline $\mathrm{HNO}_{3}$ & $277-373$ & $0-100$ & 0.9990 & 11 \\
\hline $\mathrm{NaNO}_{3}(2)$ & 288 & $0-45$ & 0.9998 & 2.7 \\
\hline $\mathrm{NaNO}_{2}$ & 288 & $0-20$ & 1.000 & 0.22 \\
\hline
\end{tabular}

The references used for data were

$\begin{array}{ll}\mathrm{NaNO}_{3} & \text { Table 3-93, Perry and Chilton (1973); p. F-5, CRC (1975) } \\ \mathrm{NaCl} & \text { Table 3-90, Perry and Chilton (1973); p. F-5, CRC (1975) } \\ \mathrm{NaOH} & \text { Table 3-92, Perry and Chilton (1973); p. F-5, CRC (1975) } \\ \mathrm{Na}_{2} \mathrm{CO}_{3} & \text { Table 3-88, Perry and Chilton (1973); p. F-5, CRC (1975) } \\ \mathrm{NaNO}_{2} & \text { Table 3-94, Perry and Chilton (1973); p. F-5, CRC (1975) } \\ \mathrm{HNO}_{3} & \text { Table 3-68, Perry and Chilton (1973); p. F-5, CRC (1975) }\end{array}$

\subsubsection{Multi-component}

Correlations are also available for the density of multi-component solutions that were designed as simulants of tank waste supernatant. For modeling tank waste, the multi-component correlations, being more specific, are preferable to the single-component correlations in Section 4.2.1. A template for multi-component correlations is given in Equation (4.4).

\subsubsection{Template for $\rho_{c}$, the liquid density $\left(\mathrm{kg} / \mathrm{m}^{3}\right)$}

$$
\rho_{c}=a_{0}+a_{1} C+a_{2} C^{3}+a_{3} T+a_{4} / T+a_{5} C T+a_{6} T^{2}+\sum_{i=1}^{N} b_{i} m_{i}
$$

Range: 277-373 K; concentration between 0 and saturation 
where $a_{k} \quad=$ coefficients obtained by data correlation

$\mathrm{m}_{\mathrm{i}} \quad=$ molality of the $\mathrm{i}$-th of $\mathrm{N}$ aqueous species (gmol/ $\mathrm{kg}$ water in solution)

$b_{i} \quad=$ density coefficient (from correlation) of the $i$-th aqueous species

$\mathrm{C} \quad=$ total concentration of all solutes in weight percent of the total solution

The density correlation can be put entirely in terms of weight percent concentrations by using the definition of molality in Equation (4.5):

$$
\mathrm{m}_{\mathrm{i}}=\frac{\mathrm{C}_{\mathrm{i}}}{\mathrm{C}_{\mathrm{H} 2 \mathrm{O}}} \frac{1000}{\mathrm{~W}_{\mathrm{i}}}
$$

where $C_{i}=$ weight percent of the $i$-th aqueous species

$\mathrm{C}_{\mathrm{H} 2 \mathrm{O}}=$ weight percent of water in the solution $=100-\mathrm{C}$

$\mathrm{W}_{\mathrm{i}}=$ molecular weight of the $\mathrm{i}$-th aqueous species

\subsubsection{Data}

Reynolds and Herting (1984) tested a set of solutions that chemically simulated the liquids found in the Hanford waste tanks to determine the solubility of the dissolved solids and the density of the solutions. Because their main concern was the near-saturation regime, they studied highconcentration conditions. The range of their experiments included temperature from 60 to $100^{\circ} \mathrm{C}$; $\mathrm{NaNO}_{2}$ concentration from 3.5 to $14.5 \mathrm{~m} ; \mathrm{NaNO}_{3}$ from 0 to $12.0 \mathrm{~m} ; \mathrm{NaOH}$ from 5.0 to $11.5 \mathrm{~m}$; and $\mathrm{NaAlO}_{2}$ from 1.0 to $3.0 \mathrm{~m}$. (Here $m$ denotes that concentration is in units of molality.) Some minor constituents of the Hanford wastes were also included in the experimental solutions; though they were not varied, they were present to provide a more accurate simulation of waste chemistry.

Because tank waste modeling requires correlations that extend to a more dilute concentration range, the present work combines the density data presented by Reynolds and Herting (1984) with single-component, low-concentration solution density data from Perry and Chilton (1973) and CRC (1975). This extended database was used to derive wide-range density correlations for Hanford waste solutions. Table 4.2 gives two versions of the wide-range density correlations, Version 1 for cases where only the total weight fraction of dissolved solids is known, and Version 2 (more accurate) that also accounts for $\mathrm{NaOH}$ concentration. 
Table 4.2. Density correlations $\left(\mathrm{kg} / \mathrm{m}^{3}\right)$ for multi-component aqueous solutions of tank waste simulants. The correlations all use the template in Equation (4.4).

\begin{tabular}{|c|c|c|c|c|c|c|}
\hline & $a_{0}$ & $a_{1}$ & $a_{3}$ & $a_{6} * 10^{3}$ & $\mathrm{~b}_{\mathrm{OH}}$ & Max. error \\
\hline Jersion 1 & 1245.8 & 9.824 & -1.0606 & 0.6812 & & $8.2 \%$ \\
\hline Version 2 & 1171.8 & 9.140 & -0.7758 & 0.4866 & 4.318 & $6.0 \%$ \\
\hline
\end{tabular}

Here $b_{\mathrm{OH}}$ is the density coefficient for the hydroxide molality; the other aqueous species had density coefficients of zero, according to the correlation. The correlation constants for the correlations in Table 4.2 are greater than $98 \%$.

A further refinement of the multi-component solution density correlation can be made, to include the effect of dissolved ammonia. Assume that ammonia reduces the multi-component solution density in the same way that it reduces the density of a single-component ammonia solution (compared to water). When data in Tables 3-32 and 3-34 of Perry and Chilton (1973) are correlated to find the temperature and concentration dependence of the ratio solution density/water density, the result is as shown in Equation (4.6).

Range: $0-8.0$ molal in $\mathrm{NH}_{3}$; temperature $273-298 \mathrm{~K}$

$$
\begin{aligned}
& \rho_{\mathrm{NH} 3} / \rho_{\text {water }}=0.9962+0.01277 \mathrm{~m}_{\mathrm{NH} 3}-6.473 \times 10^{-5} \mathrm{~m}_{\mathrm{NH} 3} \mathrm{~T} \\
& \text { where } \rho_{\mathrm{NH} 3}=\text { density of aqueous ammonia solution } \\
& \rho_{\text {water }}=\text { density of water at the same temperature } \\
& \mathrm{m}_{\mathrm{NH} 3}=\text { molality of ammonia in solution }(\mathrm{gmol} \mathrm{NH} / \mathrm{kg} \text { water) } \\
& \mathrm{T}=\quad \text { temperature }(\mathrm{K})
\end{aligned}
$$

If Equation (4.6) is multiplied by the ammonia-free multi-component solution density obtained from Table 4.2 and Equation (4.4), the density of the solution containing ammonia can be estimated. The correlation in Equation (4.6) had a correlation constant of 0.99 and a maximum error of $0.3 \%$. The error is below $5 \%$ for an ammonia molality less than or equal to $25 \mathrm{~m}$. 


\subsection{Single-phase solid density}

The solids in tank wastes include sodium nitrate, sodium nitrite, aluminum hydroxides (such as boehmite and gibbsite), other metal hydroxides, and organic compounds. Other compounds are also present in smaller quantities.

\subsubsection{Single component}

The density of the tank waste solids can be approximated as that of the single major solid species, if the predominant constituent is known.

\subsubsection{Template for $\rho_{s}$, the solid density $\left(\mathrm{kg} / \mathrm{m}^{3}\right)$}

$$
\rho_{\mathrm{s}}=\mathrm{a}_{0}
$$

Range: temperature from 273 to $373 \mathrm{~K}$

where a $\quad=$ a constant (refer to Table 4.3)

\subsubsection{Data}

The solid densities for several sodium salts were found in the CRC Handbook (1975) and are presented in Table 4.3.

Table 4.3. Densities of some solid salts present in the tank waste.

$\begin{array}{ll}\text { Salt } & \text { Density }\left(\mathrm{kg} / \mathrm{m}^{3}\right) \\ \mathrm{NaNO}_{3} & 2261 \\ \mathrm{NaNO}_{2} & 2168 \\ \mathrm{NaCl} & 2165 \\ \mathrm{Na}_{2} \mathrm{CO}_{3} & 2532 \\ \mathrm{AlOOH} & 3010 \\ \mathrm{Al}_{2} \mathrm{O}_{3} \cdot 3 \mathrm{H}_{2} \mathrm{O} \text { (gibbsite) } & 2420\end{array}$


All of these values were measured at $20^{\circ} \mathrm{C}$. No information on thermal expansion was given but, over the $30-90^{\circ} \mathrm{C}$ range in the tanks, any solid density change would probably be negligible. However, if liquid is incorporated into the sludge particles as micro-inclusions, or bound in the solids as hydrates, the effective solid density would be lower than the literature values. The measured densities for filtered solids from Tank 241-SY-101 are substantially lower than the values in Table 4.3; they range from 1700 to $2300 \mathrm{~kg} / \mathrm{m}^{3}$ (Tingey et al. 1994)(3). Because the measured densities increased with additional centrifugation, it is possible the lowest values in the range still contained some free liquid.

\subsubsection{Multi-component}

Given a composition for the solids in the tank waste, the densities can be combined to obtain an average density, as shown in Equation (4.7).

\subsubsection{Template for $\rho_{\mathrm{s}}$, the solid density $\left(\mathrm{kg} / \mathrm{m}^{3}\right)$}

$$
\rho_{s}=\frac{100}{\sum_{i=1}^{N} C_{i} / \rho_{i}}
$$

Range: all of the compositions and temperatures expected for the tanks

$$
\text { where } \begin{aligned}
\rho_{i} & =\text { the density of the } \mathrm{i}-\mathrm{th} \text { of } \mathrm{N} \text { solid species }\left(\mathrm{kg} / \mathrm{m}^{3}\right) \\
C_{i} & =\text { weight percent of the } \mathrm{i} \text {-th aqueous species }
\end{aligned}
$$

\subsubsection{Data}

The densities of some solids in tank wastes are listed in Table 4.3. The linear combination of densities is an approximation technique used where no information is known about the non-linear effect of chemical species on the overall density.

(3) Bredt, P.R. 1994. "101-SY Dilution Studies." Letter to C.W. Stewart, Pacific Northwest Laboratory, Richland, Washington. July 26, 1994.

Tingey, J.M. 1992. "Physical Characterization of Tank 101-SY Core Samples from Window C." Letter report, Pacific Northwest Laboratory, Richland, Washington. February 1992. 


\subsection{Liquid-solid (gasless) slurry density}

The sludge (settled non-convective layer) and slurry (mixed or convective layer) in the tanks are combinations of liquid, solid, and (often) gas phases. This section characterizes the density of liquid-solid slurries and sludges as a lead-in to Section 4.5 and its discussion of bubbly sludges. Equation (4.8) gives the density of the gasless slurry.

\subsubsection{Template for $\rho_{m}$, the gasless slurry density $\left(\mathrm{kg} / \mathrm{m}^{3}\right)$}

$$
\rho_{m}=\varepsilon_{c} \rho_{c}+\left(1-\varepsilon_{c}\right) \rho_{s}
$$

Range: all values of porosity

$$
\text { where } \begin{aligned}
\varepsilon_{c} & =\text { the porosity (volume fraction not occupied by solid) } \\
\rho_{c} & =\text { density of liquid phase }\left(\mathrm{kg} / \mathrm{m}^{3}\right) \\
\rho_{s} & =\text { the density of the multi-component solid }\left(\mathrm{kg} / \mathrm{m}^{3}\right)
\end{aligned}
$$

\subsubsection{Data}

A range of values has been estimated for $\varepsilon_{c}$ in the settled sludge in Tank 241-SY-101. Tables 3.7 and 3.10 in Tingey et al. (1994) give the slurry density $\rho_{m}$ and filtrate density $\rho_{c}$ for a composite sample from a gasless Window C non-convective core. Assuming a true solids density of $2200 \mathrm{~kg} / \mathrm{m}^{3}$, consistent with the intrinsic densities discussed in Section 4.3 .1 .2 , the porosity $\varepsilon_{\mathrm{c}}$ was 0.74 at $50^{\circ} \mathrm{C}$, 0.94 at $70^{\circ} \mathrm{C}$, and 0.97 at $90^{\circ} \mathrm{C}$. The data for the original core samples from the non-convective layer during Window $\mathrm{C}^{(4)}$ give the estimated porosities that are shown in Table 4.4.

(4) Tingey, J.M. 1992. "Physical Characterization of Tank 101-SY Core Samples from Window C." Letter report, Pacific Northwest Laboratory, Richland, Washington. February 1992. Tables 3.3 and 3.4 . 
Table 4.4. Porosities estimated from Tank 241-SY-101 sludge and supernatant densities.

\begin{tabular}{|c|c|c|c|c|}
\hline Segment & $32^{\circ} \mathrm{C}$ & $50^{\circ} \mathrm{C}$ & $65^{\circ} \mathrm{C}$ & $80^{\circ} \mathrm{C}$ \\
\hline 15 & 0.74 & 0.81 & 0.71 & 0.83 \\
\hline 19 & 0.74 & 0.73 & 0.71 & 0.60 \\
\hline 22 & 0.66 & 0.71 & 0.72 & 0.75 \\
\hline
\end{tabular}

When the composite core samples were subjected to longer centrifugation(5) than that used by Tingey, a density-based sludge porosity (at $50^{\circ} \mathrm{C}$ ) of 0.71 was obtained. Thus, a comparison of sludge, liquid, and solids densities suggests that in the gasless sludge the liquid occupies a fraction of 0.70 to 0.80 of the volume and the solids occupy 0.20 to 0.30 of the volume. The high porosity might be accounted for by the presence of dendritic particles that interlock and stop particle settling without allowing full compaction. For comparison, a settled sludge of kaolin contained about 20 vol\% solids (Terrones and Eyler 1993).

However, a much higher solid volume fraction (and lower porosity) is suggested by measurements of the volume fraction of centrifuged solids. Tingey et al. (1994) and other sources(6) give values from 0.62 to 0.84 for the solid volume fraction in the non-convective layer of Tank 241-SY-101 during Window C. Those values of solid fraction correspond to small porosities between 0.16 and 0.38 . The measured bulk volume of particles was probably greater than the volume of the particles, so that this method of measurement overstates the solid volume fraction in the sludge. If the maximum packing fraction of the polydisperse sludge particles was 0.8 , as for a bimodal suspension with the larger particles more than 10 times the size of the smaller (Chang and Powell 1994), then the true solid volume fraction would be between 0.50 and 0.67 , and the porosities between 0.33 and 0.50 . No data were found to resolve the gasless porosity $\varepsilon_{\mathrm{c}}$ more closely than this.

\subsection{Liquid-solid-gas (bubbly) slurry density}

The density of this type of slurry depends not only on the proportions of liquid, solid, and gas, but on the morphology of the gas. It is proposed that if dendritic bubbles grow in an initially

(5) Bredt, P.R. 1994. "101-SY Dilution Studies." Letter to C.W. Stewart, Pacific Northwest Laboratory, Richland, Washington. July 26, 1994.

(6) Tingey, J.M. 1992. "Physical Characterization of Tank 101-SY Core Samples from Window C." Letter report, Pacific Northwest Laboratory, Richland, Washington. February 1992. 
gasless slurry, they drive the liquid out of the existing pores and affect the slurry density differently than do spherical bubbles, which displace both liquid and particles. The bubble morphology is determined by a balance of the forces acting on the bubbles, as explained in Section 4.5.2. The density relationships for dendritic and spherical bubbles are shown in Equation (4.9) and (4.10), respectively.

\subsubsection{Template for $\rho_{b}$, the bubbly slurry density $\left(\mathrm{kg} / \mathrm{m}^{3}\right)$}

$$
\rho_{b}=\left(\varepsilon_{c}-\varepsilon_{d}\right) \rho_{c}+\left(1-\varepsilon_{c}\right) \rho_{s}
$$

Range: for dendritic bubbles (those that displace liquid, but not particles); $\varepsilon_{d} \leq \varepsilon_{c}$

$$
\rho_{b}=\left(1-\varepsilon_{d}\right) \rho_{m}
$$

Range: for roughly spherical bubbles (those that displace both liquid and particles)

$$
\begin{aligned}
& \text { where } \begin{array}{ll}
\varepsilon_{\mathrm{d}} & =\text { volume fraction of gas (void fraction) } \\
\rho_{\mathrm{c}} & =\text { density of liquid phase }\left(\mathrm{kg} / \mathrm{m}^{3}\right) \\
\rho_{\mathrm{s}} & =\text { the density of the multi-component solid }\left(\mathrm{kg} / \mathrm{m}^{3}\right) \\
\rho_{\mathrm{m}} & =\text { the density of the gasless sludge }\left(\mathrm{kg} / \mathrm{m}^{3}\right)
\end{array}
\end{aligned}
$$

In general, the bubbly sludge density is greater when the bubbles are dendritic than when they displace sludge.

\subsubsection{Data}

The bubble shape is believed to depend on the forces acting upon and supplied by the bubble (Gauglitz et al. 1994b). As long as a bubble is small enough to fit within a pore (of diameter $D_{p}$ ), it is spherical and can expand without either supporting sludge weight or displacing sludge volume. It is only when the equivalent spherical diameter $D$ of the bubble is greater than $D_{p}$ that it must either displace sludge or extend itself through the pores. Its internal pressure must supply the force required to support the sludge net weight and to cause the sludge to yield. When the bubble can supply enough force to oppose particle weight and inter-particle cohesion, it displaces sludge particles and takes on a roughly spherical shape (as seen in "Swiss-cheese sludge"); otherwise, the 
bubble extends fingers between the particles and assumes a dendritic shape. A dimensionless number akin to a Bond number can be derived to express the force balance and define the bubble shape (Gauglitz et al. 1994b).

The force balance requires an estimate of the force required for a single bubble to displace enough sludge for it to become spherical. Such an estimate depends on knowing the shear strength $\tau_{\mathrm{s}}$ of the sludge, the fractional linear strain $\mathrm{E}$ at which the sludge fails (flows freely), and the distance from the bubble at which that fractional failure strain is reached as a result of bubble expansion. (The fractional linear strain is defined as the absolute strain $\Delta \mathrm{L}$ divided by the original length $\mathrm{L}$. The quantity $E$ is defined as the maximum strain $\Delta \mathrm{L}_{\max }$ divided by $L$.)

A dendritic bubble that is increasing in volume because of local gas generation, and therefore attempting to expand into a spherical bubble, must expand and displace sludge outward from the central point in the sphere. At the same time the bubble pulls in its fingers from the dendritic extremities of the pore space it occupies. It expands from its initial volume, $\varepsilon_{d}$ of the volume contained in the final sphere occupied by the bubble, to $100 \%$ of that volume. That means that a volume fraction $\left(1-\varepsilon_{d}\right)$ of the bubble volume is displaced during the expansion.

The bubble can be modeled as a sphere of volume $\varepsilon_{d}$ that has expanded to a sphere with a volume of 1 ; at the final spherical bubble surface, the expansion has caused a fractional linear strain that is equal to the added diameter divided by the original diameter. So the fractional linear strain at the bubble surface equals $\left(\left(1 / \varepsilon_{d}\right)^{1 / 3}-1\right)$. At some distance from the bubble center, corresponding to a diameter $D_{E}$, the linear strain will have decreased to $E$ (and the stress will have decreased to $\tau_{s}$ ). Assume the volumetric strain is constant with distance from the bubble center. Then, the fractional linear strain varies with distance from the bubble at a rate proportional to $1 / \mathrm{r}^{2}$, giving Equation (4.11).

$$
D_{E}=D_{d} \sqrt{\frac{\left(1 / \varepsilon_{d}\right)^{1 / 3}-1}{E}}
$$


The stress at $D_{E}$ is $\tau_{s}$. This stress, multiplied by the surface area of the $D_{E}$ sphere, gives the force at the spherical surface with diameter $D_{E}$, as shown in Equation (4.12).

$$
\mathrm{F}_{\mathrm{e}}=\pi \tau_{\mathrm{s}} \mathrm{D}_{\mathrm{d}}^{2} \frac{\left(1 / \varepsilon_{\mathrm{d}}\right)^{1 / 3}-1}{\mathrm{E}}
$$

In Equation (4.12), $\mathrm{F}_{\mathrm{e}}$ is the force the bubble would have to supply to move the sludge and expand itself into a sphere.

However, this description applies only to the effect of a single isolated bubble. What of the case where a significant void fraction $\varepsilon_{\mathrm{d}}$ exists and more than one bubble at once is contained within any given sphere of diameter $D_{E}$ ? Because all these bubbles are within each other's sphere of influence (the sphere within which sludge yield occurs), the sludge displacement forces they exert will tend to add together and assist each other in displacing sludge. In each such volume of diameter $D_{E}$ there will be a number of bubbles $N_{v}$ which can be calculated by Equation (4.13).

$$
N_{v}=\frac{\pi}{6} N_{0} D_{E}^{3}=\frac{\pi}{6} N_{0} D_{d}^{3} \frac{\left(\left(1 / \varepsilon_{d}\right)^{1 / 3}-1\right)^{3 / 2}}{E^{3 / 2}}
$$

In Equation (4.13), $\mathrm{N}_{0}$ is the original, pre-gas-generation number of bubbles per unit volume; this number density is assumed to remain constant throughout bubble growth. Treating the forces applied by all these bubbles within $D_{E}$ as linearly additive, which is an extremely rough approximation, the required sludge-displacement force $F_{e b}$ per bubble is only $F_{e} / N_{v}$, or

$$
F_{e b}=\frac{6 \tau_{s} \sqrt{E}}{N_{0} D_{s}} \frac{1}{\sqrt{\left(1 / \varepsilon_{d}\right)^{1 / 3}-1}}
$$

The sludge displacement force does not depend on the depth of sludge. Disconcertingly, in Equation (4.14) the required force decreases with increasing bubble size $D_{d}$; this dependence exists because larger bubbles have larger sludge displacement volumes that contain more other bubbles to help strain the sludge. Note that the change in sludge strength $\tau_{s}$, that would be expected to result 
from the presence of a significant void fraction, is not included above. The effect of dendritic bubbles on sludge strength is expected to differ from that of sludge-displacing bubbles. (Refer to Section 8.2 for a further discussion of this topic.)

Gauglitz et al. (1994b) developed a description of the force balance for the bubble, with only the sludge weight force included. The net weight force that was applied to the bubble depended on the bubble depth $h$ and the net density of the sludge (the amount by which sludge density exceeds supernatant density). The expanding force of the bubble comes from its internal pressure increment, which depends on the liquid-gas surface tension and the pore diameter, the curvature diameter governing the liquid-gas interface. The force balance gives a weight-based $\mathrm{Bond}$ number $\mathrm{Bo}_{\mathrm{w}}$ defined by Equation (4.15).

$$
\begin{aligned}
& B o_{w}=\frac{\left(\rho_{b}-\rho_{c}\right) D_{p} g h}{4 \sigma} \\
& \text { where } \rho_{b} \quad=\text { density of the bubbly sludge }(\mathrm{kg} / \mathrm{m} 3) \\
& \rho_{\mathrm{c}} \quad=\text { density of the liquid }(\mathrm{kg} / \mathrm{m} 3) \\
& \mathrm{D}_{\mathrm{p}} \quad=\text { pore diameter }(\mathrm{m}) \\
& \mathrm{g}=\text { acceleration of gravity }(\mathrm{m} / \mathrm{s} 2) \\
& \mathrm{h}=\text { depth below top of sludge }(\mathrm{m}) \\
& \sigma=\text { liquid } / \text { gas surface tension }(\mathrm{N} / \mathrm{m})
\end{aligned}
$$

In practice, the sludge weight force would be expressed more accurately as an integral over depth of the variable sludge density.

With sludge yield forces included, the new Bond number, $\mathrm{Bo}_{\mathrm{y}}$, can be calculated by a force balance, which is detailed in Equation (4.16).

bubble lift force

(encouraging expansion)

sludge force down

(resisting expansion)

resisting yield force

$$
\begin{aligned}
& =\left(\mathrm{P}_{\mathrm{L}}+\rho_{\mathrm{c}} g h+4 \sigma / \mathrm{D}_{\mathrm{p}}\right) \mathrm{A}_{\mathrm{b}} \\
& =\left(\mathrm{P}_{\mathrm{L}}+\rho_{\mathrm{b}} g h\right) \mathrm{A}_{\mathrm{b}}
\end{aligned}
$$

$$
=\frac{6 \tau_{s} \sqrt{E}}{N_{0} D_{d}} \frac{1}{\sqrt{\left(1 / \varepsilon_{d}\right)^{1 / 3}-1}}
$$




$$
\text { where } \begin{array}{ll}
\mathrm{P}_{\mathrm{L}} & =\text { a reference pressure at the top of the sludge layer }(\mathrm{Pa}) \\
\mathrm{g} & =\text { gravitational acceleration }\left(\mathrm{m} / \mathrm{s}^{2}\right) \\
\mathrm{h} & =\text { depth below sludge surface }(\mathrm{m}) \\
\sigma & =\text { surface tension between liquid and gas }(\mathrm{N} / \mathrm{m}) \\
A_{\mathrm{b}} & =\text { contact area between the bubble and the particle }\left(\mathrm{m}^{2}\right) \\
\rho_{\mathrm{b}} & =\text { density of the bubbly sludge }(\mathrm{kg} / \mathrm{m} 3) \\
\rho_{\mathrm{c}} & =\text { density of the liquid }(\mathrm{kg} / \mathrm{m} 3) \\
\mathrm{D}_{\mathrm{p}} & =\text { pore diameter }(\mathrm{m}) \\
\mathrm{D}_{\mathrm{d}} & =\text { bubble diameter }(\mathrm{m}) \\
\mathrm{E} & =\text { fractional strain at failure }(\mathrm{m} / \mathrm{m}) \\
\tau_{\mathrm{s}} & =\text { static yield strength }(\mathrm{Pa}) \\
\varepsilon_{\mathrm{d}} & =\text { void fraction }
\end{array}
$$

When the expanding and resisting forces on the bubble are equal, $\mathrm{Bo}_{\mathrm{y}}=1$ and

$$
\left(P_{L}+\rho_{c} g h+4 \sigma / D_{p}\right) A_{b}=\left(P_{L}+\rho_{b} g h\right) A_{b}+\frac{6 \tau_{s} \sqrt{E}}{N_{0} D_{d}} \frac{1}{\sqrt{\left(1 / \varepsilon_{d}\right)^{1 / 3}-1}}
$$

As an approximation, take $A_{b}=(\pi / 4)\left(D_{d}\right)^{2}\left(1-\varepsilon_{d}\right)^{2 / 3}$. This expression accounts for the area fraction that is occupied by solid at any given value of volumetric void fraction. Dividing through by $\mathrm{A}_{\mathrm{b}}$, and remembering that $\varepsilon_{\mathrm{d}}=\pi \mathrm{N}_{0}\left(\mathrm{D}_{\mathrm{d}}\right)^{3 / 6}$, the Bond number $\mathrm{Bo}$ that includes interparticle cohesion can be expressed as

$$
\mathrm{Bo}_{\mathrm{y}}=\mathrm{Bo}_{\mathrm{w}}+\frac{\tau_{\mathrm{s}} \mathrm{D}_{\mathrm{p}} \sqrt{E}}{\sigma} \frac{1}{\varepsilon_{\mathrm{d}}\left(1-\varepsilon_{\mathrm{d}}\right)^{2 / 3} \sqrt{\left(1 / \varepsilon_{\mathrm{d}}\right)^{1 / 3}-1}}
$$

In this equation, a Bond number greater than 1.0 indicates bubble-constricting forces prevail and bubbles are dendritic [Equation (4.9) applies]. A Bond number less than 1.0 indicates the bubbles displace the sludge and are roughly spherical [Equation (4.10) applies]. 
Equation (4.17) contains several approximations that may make it more useful as a conceptual guide than a practical criterion. In this regard, the equation is probably not much more uncertain than the yield data that it uses. The yield strength $\tau_{s}$ and fractional strain at failure $E$ are discussed in Section 8.0, together with applicable data taken from Tank 241-SY-101. 


\subsection{Solubility}

The solubility of the solids in the waste tanks affects several phenomena. The density of the supernatant solution depends on the amount of solute it contains, and so is limited by the solubility of the non-aqueous species. The temperature dependence of the solubility couples the solid mass with the heat transfer conditions. When water or sodium hydroxide solution is added to a tank, the quantity of solids dissolved and the chemical composition of the remaining solids are expected to affect the slurry viscosity.

This chapter provides correlations and other submodels for the solubility of common tank waste salts in single-component solutions (Section 5.1) and in multi-component solutions (Section 5.2).

\subsection{Single component}

The relationships that can be used to estimate the single-component solubilities of some of the major salts in tank waste are provided below. As can be seen by comparing the relationships in Table 5.1 to those in Section 5.2, single-component solubilities are of dubious usefulness in tank waste modeling, but are provided for completeness. Equation (5.1) is a template for singlecomponent solubility.

\subsubsection{Template for $C_{s}$, the saturated concentration (wt \%)}

$$
C_{s}=a_{0}+a_{3} T+a_{6} T^{2}
$$

Range: liquid saturated with solute; $273-373 \mathrm{~K}$

$$
\text { where } \begin{aligned}
a_{k} & =\text { coefficients obtained by data correlation } \\
\mathrm{T} & =\text { temperature }(\mathrm{K})
\end{aligned}
$$

\subsubsection{Data}

The solubilities of some salts that are present in tank waste are given in the form of correlations or interpolation equations in Table 5.1. 
Table 5.1. Solubility relations (wt\%) for aqueous solutions of some salts. The correlations all use the template in Equation (5.1).

\begin{tabular}{|c|c|c|c|}
\hline Salt & $a_{0}$ & $a_{3}$ & $a_{6} * 10^{3}$ \\
\hline $\mathrm{KNO}_{3}$ & -402.1 & 2.148 & -2.360 \\
\hline $\mathrm{NaCl}$ & 20.36 & 0.02056 & \\
\hline $\mathrm{NaNO}_{3}$ & -18.11 & 0.2209 & \\
\hline $\mathrm{NaNO}_{2}$ & -12.92 & 0.2008 & \\
\hline
\end{tabular}

\begin{tabular}{lllll} 
Salt & $\begin{array}{l}\text { Temp. } \\
\text { range } \\
(\mathrm{K})\end{array}$ & $\begin{array}{l}\text { Conc. } \\
\text { range } \\
(\mathrm{wt} \%)\end{array}$ & $\begin{array}{c}\text { Corre- } \\
\text { lation } \\
\mathrm{r}^{2}\end{array}$ & $\begin{array}{c}\text { Max. error } \\
(\mathrm{wt} \%)\end{array}$ \\
\hline $\mathrm{KNO}_{3}$ & $273-380$ & $9-76$ & -1.00 & $0.9(9.4 \%)$ \\
$\mathrm{NaCl}$ & $273-387$ & $26-28$ & 0.97 & $0.2(0.8 \%)$ \\
$\mathrm{NaNO}_{3}$ & $273-373$ & $42-64$ & & \\
$\mathrm{NaNO}_{2}$ & $273-373$ & $42-62$ & &
\end{tabular}

The references from which data were taken were

$\mathrm{KNO}_{3} \quad$ Table 3.3.3 of Ochs et al. (1981)

$\mathrm{NaCl}$ Table 3.6.4 of Ochs et al. (1981)

$\mathrm{NaNO}_{3} \quad$ Perry and Chilton (1973), Table 3-1

$\mathrm{NaNO}_{2} \quad$ Perry and Chilton (1973), Table 3-1

Note that the relations given for $\mathrm{NaNO}_{3}$ and $\mathrm{NaNO}_{2}$ are not correlations, but linear interpolations between the temperatures of $273 \mathrm{~K}$ and $373 \mathrm{~K}$. The saturated concentration ranges for $\mathrm{NaNO}_{3}$ and $\mathrm{NaNO}_{2}$ are narrow enough to suggest the saturated concentrations can be taken as linearly dependent on temperature, as for $\mathrm{NaCl}$.

\subsection{Multi-component}

The discussion in Section 5.1 treats the tank solution as a single-component solution, although it is multi-component. More detailed composition and solubility data have been taken using a tank waste simulant (Reynolds and Herting 1984). These data allow an estimation of the 
multi-component solubility, meaning the set of compositions at which one or more of the aqueous species begins to precipitate. A template for multi-component solubility is given in Equation (5.2).

\subsubsection{Template for $s_{m}$, the saturated concentration (molal)}

$$
s_{m}=a_{0, m}+a_{3, m} t+a_{6, m} t^{2}+\sum_{i=1, i \neq m}^{N} \beta_{m, i} m_{i} t+\sum_{i=1, i \neq m}^{N} b_{m, i} m_{i}+\sum_{j=1, j \neq m}^{N} \sum_{i=1, i \neq m}^{N} d_{m, i, j} m_{i} m_{j}
$$

Range: concentrations at and below the solubility range of one or more aqueous species; temperatures $273-373 \mathrm{~K}$

$$
\begin{aligned}
& \text { where } a_{k, m}=\text { temperature coefficients of the species-m solubility } \\
& \mathrm{t} \quad=\text { temperature }\left({ }^{\circ} \mathrm{C}\right) \\
& \mathrm{m}_{\mathrm{i}} \quad=\text { molality of the } \mathrm{i} \text {-th species of } \mathrm{N} \text { aqueous species (gmol } \mathrm{i} / \mathrm{kg} \text { water) } \\
& \beta_{\mathrm{m}, \mathrm{i}}=\text { joint temperature-concentration solubility coefficients for the effect of the } \\
& b_{m, i}=\text { concentration coefficients for the effect of the } i-t h \text { species molality on the } \\
& \text { species-m solubility } \\
& \mathrm{d}_{\mathrm{m}, \mathrm{i}, \mathrm{j}}=\text { joint concentration solubility coefficients for the effect of the combined } \\
& \mathrm{i} \text {-th and } \mathrm{j} \text {-th species molalities on the species-m solubility }
\end{aligned}
$$

Molality is defined as gmol of a species/ $\mathrm{kg}$ water.

\subsubsection{Data}

To obtain solubility correlations with a wide concentration range, the high-concentration simulant solubility data from Reynolds and Herting (1984) were combined with single-component solubility data for $\mathrm{NaNO}_{3}$ and $\mathrm{NaNO}_{2}$ (Table 3-1, Perry and Chilton, 1973). Owing to a lack of wellconfirmed single-component $\mathrm{NaAlO}_{2}$ data to use as a basis for extended re-correlation, the Reynolds and Herting correlation for $\mathrm{NaAlO}_{2}$ solubility was used as is. The resulting correlations for aqueous species are given in Table 5.2. 
Table 5.2. Solubility relations $(w t \%)$ for the major salts in waste simulant solutions. The correlations all use the template in Equation (5.2).

\begin{tabular}{|c|c|c|c|c|c|}
\hline Species $\mathrm{m}$ & $a_{0, m}$ & $a_{3, m}$ & $a_{6, m} * 10^{3}$ & $\beta_{\mathrm{m}, \mathrm{OH}}$ & $b_{\mathrm{m}, \mathrm{OH}}$ \\
\hline$\left(\mathrm{NO}_{3}\right)^{-}$ & 12.59 & -0.005538 & 0.9820 & & -1.928 \\
\hline$\left(\mathrm{NO}_{2}\right)^{-}$ & 16.90 & -0.08353 & 1.637 & & -1.966 \\
\hline$\left(\mathrm{AlO}_{2}\right)^{-}$ & 9.359 & -0.0899 & 6.29 & 0.00304 & -0.567 \\
\hline
\end{tabular}

\begin{tabular}{|c|c|c|c|c|c|}
\hline Species m & $b_{\mathrm{m}, \mathrm{NO} 2}$ & $b_{\mathrm{m}, \mathrm{NO} 3}$ & $\mathrm{~d}_{\mathrm{m}, \mathrm{OH}, \mathrm{OH}}$ & $\mathrm{d}_{\mathrm{m}, \mathrm{NO} 2, \mathrm{NO} 2}$ & $\mathrm{~d}_{\mathrm{m}, \mathrm{NO} 3, \mathrm{NO} 3}$ \\
\hline$\left(\mathrm{NO}_{3}\right)^{-}$ & -0.2806 & & 0.09675 & 0.01957 & \\
\hline$\left(\mathrm{NO}_{2}\right)^{-}$ & & -0.0549 & 0.09188 & & 0.01828 \\
\hline
\end{tabular}

$\left(\mathrm{AlO}_{2}\right)$

Species $m \quad d_{m, N O 2, N O 3}$

$\left(\mathrm{NO}_{3}\right)^{-}$

$\left(\mathrm{NO}_{2}\right)^{-}$

$\left(\mathrm{AlO}_{2}\right)^{-}$

$-0.00534$

Temp.

range

Species $\mathrm{m}$

$\left(\mathrm{NO}_{3}\right)$

$\left(\mathrm{NO}_{2}\right)$

$\left(\mathrm{AlO}_{2}\right)^{-}$
(K)

$273-373$

$273-373$

$333-373$
Conc.

range Max. error

$(m)$

(m)

$\mathrm{NO}_{2}$ 0-14.5, $\mathrm{OH} \mathrm{0-11.5,} \mathrm{AlO}_{2}$ 0-3.0 $0.70(14 \%)$

$\mathrm{NO}_{3} 0-12, \mathrm{OH} 0-11.5, \mathrm{AlO}_{2}$ 0-3.0 1.27 (11\%)

$\mathrm{NO}_{3} 0-12, \mathrm{NO}_{2} 0-14.5, \mathrm{OH} 0-11.51 .18(28 \%)$

The correlations were carried out with temperatures of ${ }^{\circ} \mathrm{C}$ rather than $\mathrm{K}$ to avoid the errors inherent in estimating the molality as a small difference between large numbers.

These correlations were compared with average chemical composition data calculated from composite samples of Tank 241-SY-101 contents (Bredt et al. 1994). The water concentration was 
$35.5 \mathrm{wt} \%, \mathrm{OH} 2.47 \mathrm{wt} \%, \mathrm{NO}_{3} 11.7 \mathrm{wt} \%, \mathrm{NO}_{2} 10.5 \mathrm{wt} \%$, and $\mathrm{Al} 3.2 \mathrm{wt} \%$. As a first approximation, if each of these salts is assumed totally dissolved in the water, the solution is calculated to be $4.1 \mathrm{~m}$ in $\mathrm{OH}, 5.3 \mathrm{~m}$ in $\mathrm{NO}_{3}, 6.4 \mathrm{~m}$ in $\mathrm{NO}_{2}$, and $3.3 \mathrm{~m}$ in $\mathrm{Al}$. At these coricentrations, the solubility correlations in Equation (5.2) and Table 5.2 predict $50^{\circ} \mathrm{C}$ solubilities of $7.5 m$ in $\mathrm{NO}_{3}, 10.5 \mathrm{~m}$ in $\mathrm{NO}_{2}$, and $4.5 \mathrm{~m}$ in Al. In short, the correlations indicate the salts in Tank SY-101 should be entirely dissolved as do the original Reynolds and Herting (1984) correlations, though those give somewhat lower solubilities]. However, observations show substantial undissolved solids are present in the tank.

The reason for the disagreement between the SY-101 measurements and the correlations is not clear. The Reynolds and Herting (1984) correlations have been used for some time to predict evaporator performance, have received substantial in-use verification, and would be expected to provide reasonably accurate predictions. Perhaps some of the water measured by chemical analyses was water of hydration; if less free water were present, the amount of mass that could be dissolved in it would decrease. Possibly, the minor constituents in the waste (for example, ammonia, which was not included in the 1984 simulant experiments) have a greater effect on the solubility of major constituents than would be expected. Additional simulant experimental data might resolve this issue.

Another approach, one that is not amenable to description as a template, has also been used to provide a submodel for tank waste solubility. In this technique, the Gibbs free energy of a mixture of solid, solution, and gas phases is minimized to find the equilibrium solubilities of all of the components of the mixture. The GMIN code (Felmy 1990) is one example of a computer submodel that uses the free energy minimization method, and that contains a database of the standard chemical potentials and Pitzer ion-interaction parameters needed to model the chemical components of the tank waste. In tank waste applications, GMIN simulates such chemical reactions as those listed in Equations (5.3) through (5.9).

$$
\begin{aligned}
& \mathrm{Na}^{+}+\mathrm{NO}_{3^{-}}=\mathrm{NaNO}_{3}(\mathrm{aq}) \\
& \mathrm{Na}^{+}+\mathrm{NO}_{2^{-}}=\mathrm{NaNO}_{2}(\mathrm{aq}) \\
& \mathrm{Na}^{+}+\mathrm{NO}_{3^{-}}=\mathrm{NaNO}_{3}(\mathrm{~s}) \\
& \mathrm{Na}^{+}+\mathrm{NO}_{2^{-}}=\mathrm{NaNO}_{2}(\mathrm{~s}) \\
& \mathrm{Al}(\mathrm{OH})_{4^{-}}\left(+\mathrm{Na}^{+}\right)=\mathrm{OH}^{-}+\mathrm{Al}(\mathrm{OH})_{3}(\mathrm{~s})\left(+\mathrm{Na}^{+}\right) \\
& \mathrm{Al}(\mathrm{OH})_{4^{-}}\left(+\mathrm{Na}^{+}\right)=\mathrm{OH}^{-}+\mathrm{H}_{2} \mathrm{O}+\mathrm{AlOOH}(\mathrm{s})\left(+\mathrm{Na}^{+}\right) \\
& \mathrm{Na}++\mathrm{Al}(\mathrm{OH})_{4^{-}}=\mathrm{NaAlO}_{2}(\mathrm{~s})+2 \mathrm{H}_{2} \mathrm{O} \\
& \mathrm{Na}^{+}+\mathrm{AlO}_{2^{-}}=\mathrm{NaAlO}_{2}(\mathrm{~s})
\end{aligned}
$$


The GMIN code is currently being incorporated as a submodel in the TEMPEST thermal fluid dynamics model(7). GMIN will be used to calculate equilibrium conditions for those species that are not rate limited. It will also supply activity products and equilibrium constants, which will then be used as the driving force term in kinetics equations that describe dissolution and precipitation as a combination of zero-order and first-order processes. The differential equations for the solid species are posed in the general form shown in Equation (5.11).

$$
\begin{aligned}
& \mathrm{d}\left(\mathrm{m}_{\mathrm{i}}\right) / \mathrm{dt}=\left(\mathrm{k}_{0}+\mathrm{k}_{1} \mathrm{~m}_{\mathrm{i}}\right)\left(1-\mathrm{Q}_{\mathrm{i}} / \mathrm{K}_{\mathrm{i}}\right) \\
& \text { where } \mathrm{m}_{\mathrm{i}}=\text { molality of the } \mathrm{i} \text {-th solid species in suspension (gmol } \mathrm{i} / \mathrm{kg} \text { water) } \\
& \mathrm{k}_{0}, \mathrm{k}_{1}=\text { dissolution/precipitation rate constants for solid species } \mathrm{i} \\
& \mathrm{Q}_{\mathrm{i}}=\text { activity product for species } \mathrm{i} \text { (supplied by GMIN) } \\
& \mathrm{K}_{\mathrm{i}}=\text { equilibrium constant for species } \mathrm{i} \text { (supplied by GMIN) }
\end{aligned}
$$

GMIN (or a similar code) could also be used to generate solubility values for mixtures; hypothetically, the code output could be correlated to provide relatively simple equation forms [such as Equation (5.2)] that could be used for manual or spreadsheet solubility calculations.

(7) Brothers, J.W., et al. 1995. "Flammable Gas Project -- February Status Report." Monthly report to Westinghouse Hanford Company from Pacific Northwest Laboratory, Richland, Washington. March 14, 1995. 


\subsection{Vapor Pressure}

The vapor pressures of water and ammonia affect tank waste processing and modeling. The vapor pressure of water helps control the evaporation rate, and thus the cooling rate, of wastes in some of the Hanford tanks. The ammonia vapor pressure determines the release rate of ammonia, a chemical of concern because of its toxicity.

This chapter provides correlations and other submodels for the vapor pressure of water and ammonia in single-component solutions (Section 6.1) and in multi-component solutions (Section 6.2).

\subsection{Single component}

The relationships that can be used to estimate the water and ammonia vapor pressures in sodium hydroxide solution (a single-component solution) are provided in Section 6.1.1. A comparison of the relationships in Table 6.1 with those in Section 6.2 shows single-component vapor pressures are of dubious usefulness in tank waste modeling. They are provided for completeness. A single-component vapor pressure relationship is given in Equation (6.1).

\subsubsection{Template for $P_{v}$, the vapor pressure $(\mathrm{Pa})$}

$$
P_{v}=\left(m_{i}\right) \beta \exp \left(a_{0}+a_{1} C+a_{2} C^{2}+a_{4} / T\right)
$$

Range: 298-343 K; 0-22 wt\% $\mathrm{NaOH} ; 0-3 m \mathrm{NH}_{3}$

$$
\begin{aligned}
& \text { where } \mathrm{m}_{\mathrm{i}} \quad=\text { molality of aqueous species or water, gmol of species } / \mathrm{kg} \text { water } \\
& \text { B . = concentration exponent for the species, from correlation } \\
& a_{k}=\text { coefficients obtained by data correlation } \\
& \mathrm{C}=\text { total concentration of all solutes in weight percent of the total solution } \\
& \mathrm{T}=\text { temperature }(\mathrm{K})
\end{aligned}
$$

\subsubsection{Data}

The vapor pressures of water and ammonia dissolved in $\mathrm{NaOH}$ solutions are given as correlations in Table 6.1. 
Table 6.1. Vapor pressures of water and ammonia $(\mathrm{Pa})$ in $\mathrm{NaOH}$ solution. The correlations use the template in Equation (6.1).

\begin{tabular}{|c|c|c|c|c|c|}
\hline Species & $a_{0}$ & $a_{1}$ & $a_{2} * 10^{3}$ & $a_{4}$ & $\beta$ \\
\hline $\mathrm{H}_{2} \mathrm{O}$ & 25.283 & -0.01775 & 0 & -5122 & 0 . \\
\hline $\mathrm{NH}_{3}$ & 19.220 & 0.06899 & 0.392 & -3511 & 1.056 \\
\hline
\end{tabular}

\begin{tabular}{lllll} 
& $\begin{array}{l}\text { Temp. } \\
\text { range } \\
\text { Species }\end{array}$ & $\begin{array}{l}\text { Conc. } \\
\text { range } \\
(\mathrm{K})\end{array}$ & $\begin{array}{l}\text { Corre- } \\
\text { lation }\end{array}$ & $\begin{array}{c}\text { Max. error } \\
(\mathrm{Pa})\end{array}$ \\
\hline $\mathrm{H}_{2} \mathrm{O}$ & $\frac{\mathrm{r}^{2}}{298-343}$ & $\overline{0-22}$ & -1.00 & $\frac{1200(12 \%)}{\mathrm{NH}_{3}}$ \\
\cline { 5 - 5 } & $298-343$ & $0-22$ & 0.99 & $4500(19 \%)$
\end{tabular}

The references from which data were taken were
$\mathrm{H}_{2} \mathrm{O}$ Tables A.1-A.7, A.9-A.12 of Norton and Pederson (1994); Table 3-21 of Perry and Chilton (1973)
$\mathrm{NH}_{3}$ Tables A.7, A.9-A.12 of Norton and Pederson (1994); Table 3-23 of Perry and Chilton (1973)

One data set, the partial pressures for $1.00 \mathrm{~m} \mathrm{NaOH}$ and $0.549 \mathrm{~m} \mathrm{NH}_{3}$ in Table A.8 of Norton and Pederson (1994), consistently proved impossible to fit into a simple correlation and was, therefore, excluded. For this data set, the correlations in Table 6.1 overestimate the water partial pressure by $37 \%$ (at all temperatures) and underestimate the ammonia partial pressure by $30-60 \%$ (depending on temperature). The correlations perform fairly well for the remaining data sets, as described in Table 6.1.

\subsection{Multi-component}

The previous discussion treats the tank solution as a single-component solution, although it is multi-component. Recent experiments with tank waste simulants (Norton and Pederson 1994) have provided estimates for the partial pressures of water and ammonia in the multi-component tank waste simulants. Of the two simulants. tested for Tank 241-SY-101, SY1-SIM-93B was homogeneous (contained no undissolved solids) and SY1-SIM-91A was heterogeneous, a suspension rather than a clear solution. Equation (6.2) is a template for species vapor pressures in multi-component solutions. 
6.2.1 Template for $P_{v}$, the vapor pressure $(\mathbf{P a})$

$P_{v}=\left(m_{i}\right)^{\beta} \exp \left(a_{0}+a_{1} C+a_{2} C^{2}+a_{4} / T+a_{5} C T\right)$

Range: $298-343 \mathrm{~K} ; 0-61 \mathrm{wt} \%$ solutes and solids; 0-3.5m $\mathrm{NH}_{3}$

where $m_{i} \quad=$ molality of aqueous species or water, gmol of species $/ \mathrm{kg}$ water

B $=$ concentration coefficient for the species, from correlation

$a_{k} \quad=$ coefficients obtained by data correlation

C = total concentration of all solutes, plus all undissolved solids, in weight percent of the total suspension

$\mathrm{T} \quad=$ temperature $(\mathrm{K})$

\subsubsection{Data}

The partial pressure correlations for the two SY-101 simulants are given in Table 6.2. Both simulants could be correlated with a single equation, although one contained undissolved solids and the other did not.

Table 6.2. Vapor pressures of water and ammonia (Pa) for two SY-101 simulants. The correlations use the template in Equation (6.2).

\begin{tabular}{|c|c|c|c|c|c|}
\hline Species & $a_{0}$ & $a_{1}$ & $a_{2} * 10^{3}$ & $a_{4}$ & $a_{5} * 10^{3}$ \\
\hline $\mathrm{H}_{2} \mathrm{O}$ & 25.506 & -0.001601 & -0.2267 & -5207 & 0 \\
\hline $\mathrm{NH}_{3}$ & 21.867 & 0.07933 & 0.4950 & -4149 & -0.2769 \\
\hline
\end{tabular}

\begin{tabular}{ll} 
Species & $\beta$ \\
\hline $\mathrm{H}_{2} \mathrm{O}$ & 0 \\
$\mathrm{NH}_{3}$ & 0.8028
\end{tabular}


Table 6.2. (continued)

\begin{tabular}{|c|c|c|c|c|}
\hline Species & $\begin{array}{c}\text { Temp. } \\
\text { range } \\
(\mathrm{K})\end{array}$ & $\begin{array}{l}\text { Conc. } \\
\text { range } \\
(w t \%)\end{array}$ & $\begin{array}{c}\text { Corre- } \\
\text { lation } \\
\mathbf{r}^{2}\end{array}$ & $\begin{array}{l}\text { Max. error } \\
(\mathrm{Pa})\end{array}$ \\
\hline $\mathrm{H}_{2} \mathrm{O}$ & $298-343$ & $0-61$ & 1.00 & $2200(15 \%)$ \\
\hline $\mathrm{NH}_{3}$ & $298-343$ & $0-61$ & 0.99 & $4500(13 \%)$ \\
\hline
\end{tabular}

The references from which data were taken are

$\mathrm{H}_{2} \mathrm{O}$ Tables B.1-B.9 of Norton and Pederson (1994); Table 3-21 of Perry and Chilton (1973)

$\mathrm{NH}_{3}$ Tables B.1-B.9 of Norton and Pederson (1994); Table 3-23 of Perry and Chilton (1973)

Because these correlations cover both simulants, they must be used with some caution: the concentration $C$ in Equation (7.2) must include all the non-water species present, whether or not they are fully dissolved. Both solute and precipitate must be included in the weight percent. Also note the data contained only three levels of concentration $\mathrm{C}$ and the correlation is quadratic in $\mathrm{C}$ : thus the coefficients of $\mathrm{C}$ are fully determined, and the quadratic dependence on $\mathrm{C}$ is not proved. 


\subsection{Surface Tension}

The surface tension of the liquid/gas interface affects the bubble rise velocity in some bubble rise regimes; Section 12 contains more details of this effect. Surface tension also helps determine the shapes of bubbles trapped in sludge, as noted in Section 4.5.2.

This chapter provides correlations and other submodels for the surface tension of singlecomponent solutions (Section 7.1) and multi-component solutions (Section 7.2). The latter section also includes information pertinent to estimating surface tension in multi-component suspensions of particles in solution.

\subsection{Single-component solution}

The supernatant liquids in the waste tanks are multi-component solutions but, in cases where the solution composition is unknown, it may be necessary to consider them as solutions of the single dominant solute. This approach is shown in Equation (7.1).

\subsubsection{Template for $\sigma$, the surface tension $(\mathrm{N} / \mathrm{m})$}

$\sigma=a_{0}+a_{1} C+a_{2} C^{2}+a_{3} T+a_{5} C T$

Range: $283-353 \mathrm{~K}$ (except for $\mathrm{NaCl}$ ); less than saturated concentration

$$
\text { where } \begin{aligned}
a_{k} & =\text { coefficients obtained by data correlation } \\
\mathrm{C} & =\text { concentration of solute in weight percent of the total solution } \\
\mathrm{T} & =\text { temperature }(\mathrm{K})
\end{aligned}
$$

\subsubsection{Data}

The surface tensions of three sodium salts that are present in tank waste are given in the form of correlations in Table 7.1. With the exception of $\mathrm{NaCl}$, all of the salt solution surface tensions were correlated over a range of temperatures. For sodium chloride, data were available only at $20^{\circ} \mathrm{C}$; but note at $20^{\circ} \mathrm{C}$ the surface tensions of $\mathrm{NaOH}$ and $\mathrm{NaCl}$ are almost equal (at equal weight percent solute). 
Table 7.1. Surface tension correlations $(\mathrm{N} / \mathrm{m})$ for aqueous solutions of some sodium salts. The correlations all use the template in Equation (7.1).

\begin{tabular}{|c|c|c|c|c|c|}
\hline Salt & $a_{0}$ & $a_{1} * 10^{3}$ & $a_{2} * 106$ & $a_{3} * 10^{3}$ & $a_{5} * 106$ \\
\hline $\mathrm{NaNO}_{3}$ & 0.1236 & 0.4977 & 3.614 & -0.1721 & -1.446 \\
\hline $\mathrm{NaOH}$ & 0.1271 & 0.6209 & 17.84 & -0.1826 & -1.056 \\
\hline $\mathrm{NaCl}$ & 0.07282 & 0.2646 & 4.234 & & \\
\hline
\end{tabular}

\begin{tabular}{|c|c|c|c|c|}
\hline Salt & $\begin{array}{l}\text { Temp. } \\
\text { range } \\
(\mathrm{K})\end{array}$ & $\begin{array}{l}\text { Conc. } \\
\text { range } \\
(w t \%)\end{array}$ & $\begin{array}{l}\text { Corre- } \\
\text { lation } \\
r^{2}\end{array}$ & $\begin{array}{l}\text { Max. error } \\
(\mathrm{N} / \mathrm{m})\end{array}$ \\
\hline $\mathrm{NaNO}_{3}$ & $283-353$ & $0-47$ & 0.96 & $0.0028(4.7 \%)$ \\
\hline $\mathrm{NaOH}$ & $283-353$ & $0-17$ & 0.99 & $0.0014(2.0 \%)$ \\
\hline $\mathrm{NaCl}$ & 293 & $0-26$ & 1.00 & $0.0002(0.2 \%)$ \\
\hline
\end{tabular}

The references from which data were taken were

$\mathrm{NaNO}_{3}$. Table C.2 of Norton and Pederson (1994); p. F-41, CRC (1975)

$\mathrm{NaOH}$ Table C.1 of Norton and Pederson (1994)

$\mathrm{NaCl} \quad$ p. F-41, CRC (1975)

\subsection{Multi-component solution}

Experiments have been conducted to find the surface tension of Tank 241-SY-101 sludge and solution simulants (Norton and Pederson 1994). Of the two simulants tested, SY1-SIM-93B was homogeneous (that is, a solution without any precipitate) and SY1-SIM-91A, which was more concentrated, was heterogeneous, a suspension rather than a clear solution. A template for the surface tension of a multi-component solution is given in Equation (7.2).

7.2.1 Template for $\sigma$, the surface tension $(\mathrm{N} / \mathrm{m})$

$$
\sigma=a_{0}+a_{1} C+a_{3} T+\sum_{i=1}^{N} b_{i} m_{i}+C^{2} \sum_{i=1}^{N} d_{i} m_{i}
$$

Range: $303-340 \mathrm{~K}$; up to saturated concentration 

where $a_{k}=$ coefficients obtained by data correlation
$\mathrm{m}_{\mathrm{i}} \quad=$ molality of the $\mathrm{i}$-th of $\mathrm{N}$ aqueous species (gmol/ $\mathrm{kg}$ water in solution)
$b_{i} \quad=$ coefficients (from correlation) of the $i$-th aqueous species
$\mathrm{d}_{\mathrm{i}} \quad=$ coefficients (from correlation) of the $\mathrm{i}$-th aqueous species
C = total concentration of all solutes, plus undissolved solids, in weight percent of the total suspension

\subsubsection{Data}

The surface tension correlations for the two SY-101 simulants are given in Table 7.2; they are based on data taken from Tables C. 3 and C. 4 of Norton and Pederson (1994). Interestingly, it was possible to correlate both of the simulants with a single equation, even though one contained undissolved solids and the other did not.

Table 7.2. Surface tension correlations $(\mathrm{N} / \mathrm{m})$ for two $S Y-101$ waste simulants. The correlations all use the template in Equation (7.2).

$\begin{array}{lllllll}\text { Simulant } & a_{0} & & a_{1} * 103 & a_{3} * 10^{3} & b_{\mathrm{NH} 3} * 10^{3} & d_{\mathrm{NH} 3} * 106 \\ \text { SY1-SIM-93B } & 0.1246 & 0.3151 & -0.1743 & -3.308 & \\ \text { both simulants } & 0.1393 & 0.5056 & -0.2247 & -1.086 & -4.753\end{array}$

\begin{tabular}{|c|c|c|c|c|c|}
\hline Simulant & $\begin{array}{l}\text { Temp. } \\
\text { range } \\
\text { (K) }\end{array}$ & $\begin{array}{l}\text { Conc. } \\
\text { range } \\
(w t \%)\end{array}$ & $\begin{array}{l}\text { NH3 } \\
\text { range } \\
\text { (molal) }\end{array}$ & $\begin{array}{c}\text { Corre- } \\
\text { lation } \\
r^{2}\end{array}$ & $\begin{array}{l}\text { Max. error } \\
(\mathrm{N} / \mathrm{m})\end{array}$ \\
\hline IM-93B & $303-333$ & $0-31$ & $0-1.8$ & 0.97 & $0.0028(2.6 \%)$ \\
\hline both simulants & $303-340$ & $0-61$ & $0-1.8$ & 0.93 & $0.0062(7.8 \%)$ \\
\hline
\end{tabular}

In Table $7.2, b_{\mathrm{NH} 3}$ and $d_{\mathrm{NH} 3}$ are the coefficients for ammonia, the only aqueous species whose concentration was individually varied in the experiments.

The correlation that covers both simulants must be used with one caution: the concentration $\mathrm{C}$ in equation (7.2) must include all the non-water species present, whether or not they are fully dissolved. It must include both solute and precipitate. The correlation for the homogeneous simulant SY1-SIM-93B uses C to refer to only the solute, as no solids were present. 
The two simulants were entirely inorganic; tests were conducted to find out whether the organic chemicals that were known to be present in the waste, in small quantity, affected the surface tension. Table C.5 of Norton and Pederson (1994) shows the small effect of adding $1.5 \mathrm{wt} \%$ total organic carbon (TOC) to the homogeneous simulant SY1-SIM-93B. The TOC was added in the form of one of several different organics present in the tank waste: sodium citrate, sodium formate, glycine, EDTA, or HEDTA. None of these TOC chemicals had more than a $5 \%$ effect on surface tension, an amount similar to the correlation error.

Another set of experiments (Norton and Pederson 1994) concluded the nature of the gas in the bubble $\left(\mathrm{N}_{2}, \mathrm{O}_{2}, \mathrm{H}_{2}, \mathrm{~N}_{2} \mathrm{O}\right.$, or $\left.\mathrm{CH}_{4}\right)$ had no measurable effect on the surface tension. 


\subsection{Yield Strength}

The yield strength of the sludge in waste tanks determines how much stress the material can carry while continuing to behave as a solid -- that is, to undergo bounded deformation. Yield strength may affect the rollover behavior, in that the upward force exerted by the buoyant underlayer must break through the layer of sludge above. Yield strength also may affect the morphology of bubbles within the sludge (as discussed in Section 4.5 ).

As Husband et al. (1993) pointed out, three yield stresses characterize a material's rheological behavior. These characteristic stresses are 1) the elastic-limit yield stress; 2) the static yield stress; and 3) the dynamic yield stress. The elastic-limit and static yield stresses are the minimum stresses needed to cause permanent but bounded deformation, and unbounded deformation, respectively. Both of these quantities are measured with constant-stress techniques. The third quantity, the dynamic yield stress, is found from constant-strain-rate measurements and is the quantity used in rheological models. Husband gives examples of cases in which suspensions had distinctly different static and dynamic yield stresses. This chapter discusses the yield strength of gasless and bubbly sludges, and treats it as synonymous with the static yield strength of a solid material. (For a discussion of the dynamic yield stress, a fluid rheological property of the sludge, see Chapter 9.)

This chapter begins by discussing submodels for the gasless sludge yield strength in Section 8.1; Section 8.2 describes the bubbly sludge yield strength.

\subsection{Liquid-solid (gasless) slurry yield strength}

The forces that cause an agglomerate to have a yield strength can be subdivided into solidsolid forces, such as those of cohesion and particle bridging, and those forces derived from gas-liquid surface tension effects. In a gasless slurry, only the solid-solid forces are pertinent.

\subsubsection{Single-component}

For a single-component approach, the chemistry of the sludge is not a consideration and the relative amounts of solid and liquid are the composition variables of concern. A template for the yield strength of a gasless slurry is given in Equation (8.1). 


\subsubsection{Template for $\tau_{s}$, the gasless sludge yield strength $(\mathbf{P a})$}

$$
\tau_{\mathrm{s}}=\mathrm{K}\left(\varepsilon_{\mathrm{s}}\right)^{\mathrm{n}}
$$

Range: from $\varepsilon_{\mathrm{s}}=0$ to $\varepsilon_{\mathrm{s}}=0.8$; temperature from 295 to $353 \mathrm{~K}$

$$
\text { where } \begin{aligned}
\mathrm{K} & =\text { consistency coefficient (from correlation) } \\
\varepsilon_{\mathrm{s}} & =\text { solids concentration in volume fraction } \\
\mathbf{n} & =\text { exponent of concentration dependence (from correlation) }
\end{aligned}
$$

\subsubsection{Data}

The data base for yield strength correlations consists of data taken from core samples from Tank 241-SY-101. It is expected that most of the gas present in the samples escaped during sample preparation and testing; therefore, these data are considered to represent the gasless sludge.

The data for the yield strength of the non-convective layer in the Window $\mathrm{C}$ and Window $\mathrm{E}$ core samples from SY-101 were correlated versus the temperature and the centrifuged solids concentrations measured in the same set of experiments( ${ }^{(8)}$. Table 8.1 gives the yield strength correlations at the four temperatures where data were taken.

Table 8.1. Static yield strength of SY-101 sludge $(\mathrm{Pa})$. All correlations employ the template in Equation (8.1).

Temperature

(K)

295
323
338
353

$\mathbf{K}$

18300

2330

2383

2290 n

5.78

3.08

2.47

2.29
Correlation

$\mathbf{r}^{2}$

0.81

0.56

0.55

0.80

(8) Tingey, J.M. 1992. "Physical Characterization of Tank 101-SY Core Samples from Window C." Letter report, Pacific Northwest Laboratory, Richland, Washington. February 1992. Tingey, J.M. 1992. "Physical Measurements of Waste from Tank 241-SY-101 Window E." Letter report to Westinghouse Hanford Company, Pacific Northwest Laboratory, Richland, Washington. June 1992. 
The data exhibited considerable scatter. General trends, toward increase of yield strength with solids concentration, and decrease of yield strength with increasing temperature, were fairly clear; but yield strengths measured at nearly the same temperature and solids concentration usually differed by a factor of 2 to 3 . Hence, the correlation errors are also a factor of 2 .

It seems probable that conditions besides total solids and temperature affected the yield strength. One significant variable might be the particle size and shape distribution; another, the shear history of the material. The data correlated in Table 8.1 were measurements made on nonhomogenized core samples taken from different depths in the tank. Data that were taken later from homogenized samples (Tingey et al. 1994) showed much lower shear strengths. The yield strengths measured for unhomogenized Window $\mathrm{C}$ non-convective layer samples at $323 \mathrm{~K}$ ranged from 640 to $2640 \mathrm{~Pa}$; for comparison, the yield strengths for homogenized samples were 170 to $440 \mathrm{~Pa}$. Tingey et al. (1994) commented that the decrease in yield strength appeared to be irreversible over significant time periods. Presumably, solid-solid bonds were broken during homogenization and are not rapidly re-formed under sample conditions.

\subsubsection{Multi-component}

No data have been found to support correlations to describe multi-component effects on the gasless sludge yield strength.

\subsection{Liquid-solid-gas (bubbly) slurry yield strength}

The yield strength of the sludge can vary substantially as the result of the presence of a significant void fraction. The nature of that variation is expected to depend on whether the bubbles are spherical (particle-displacing) or dendritic. (Bubble morphology is discussed in Section 4.5.)

\subsubsection{Single-component}

In the single-component submodel, the yield strength is treated as a function of the relative amounts of solid, liquid, and gas, and the chemical composition of the phases is not considered. Equations (8.2) and (8.3) give templates for the yield strength of slurries and sludges containing dendritic and spherical bubbles. 
8.2.1.1 Templates for $\tau_{\mathrm{sb}}$, the bubbly sludge yield strength (Pa)

$$
\tau_{s b}=\left(\frac{\varepsilon_{s}}{1-\varepsilon_{s}}\right) \frac{\sigma C_{1} S_{b}}{D_{s}}\left(\frac{S}{S_{b}^{2}+S^{2}}-\frac{S_{a}}{S_{b}^{2}+S_{a}^{2}}\right)+\left(\frac{\varepsilon_{s}}{1-\varepsilon_{s}}\right) \frac{\sigma C_{2}}{D_{s}}\left(S-S_{b}\right)^{\alpha}\left(S_{a}-S\right)^{\alpha\left(S_{a}-S_{c}\right) / s_{z}}
$$

Range: for dendritic bubbles (those which displace liquid but not particles)

$$
\begin{aligned}
& \text { where } \sigma \\
& \begin{array}{ll}
D_{\mathrm{s}} & =\text { surface tension }(\mathrm{N} / \mathrm{m}) \\
\mathrm{S} & =\text { saturation (fraction of non-solid volume occupied by liquid) } \\
\mathrm{S}_{\mathrm{a}} & =\text { saturation at which imbibition ends } \\
\mathrm{S}_{\mathrm{b}} & =\text { saturation at which peak pendular strength occurs } \\
\mathrm{S}_{\mathrm{c}} & =\text { saturation at which peak capillary strength occurs } \\
\alpha & =\text { fitting parameter for capillary pressure } \\
\mathrm{C}_{1} & =\text { fitting parameter for maximum capillary strength } \\
\mathrm{C}_{2} & =\text { fitting parameter for maximum pendular strength } \\
\tau_{\mathrm{sb}}=\frac{4 \sigma \varepsilon_{\mathrm{d}}}{\sqrt{2} \mathrm{D}_{\mathrm{d}}}\left(\sqrt{4 \mathrm{E}^{2}+1}-1\right)+\left(1-\varepsilon_{\mathrm{d}}\right) \tau_{\mathrm{s}}
\end{array}
\end{aligned}
$$

Range: for particle-displacing (spherical) bubbles

$$
\begin{aligned}
& \text { where } \sigma \\
& \begin{array}{ll}
\varepsilon_{\mathrm{d}} & =\text { surface tension }(\mathrm{N} / \mathrm{m}) \\
\mathrm{D}_{\mathrm{d}} & =\text { gas volume fraction } \\
\mathrm{E} & =\text { ductility of the sludge (fractional strain at the yield point) } \\
\tau_{\mathrm{s}} & =\text { yield stress of the gasless sludge }(\mathrm{Pa})
\end{array}
\end{aligned}
$$




\subsubsection{Data}

No data were found to estimate the yield strength of Hanford waste sludges in which significant bubbles are present, but the literature does contain information that helps to establish the correlation templates for the property.

In the dendritic bubble regime, the gas is found entirely within the pores of the wet solid. Several yield strength experiments have been conducted on agglomerates in which the gas is found in the pores [Gauglitz et al. (1994), Pietsch et al. (1969), Schubert (1975), Schubert et al. (1975), Schubert (1977), and Leavell and Peters (1987)]. In all of these experiments, no gas was generated in the agglomerate and dendritic bubble morphology was present. Figures 8.1 and 8.2, which are based on data from Figures 6 and 7 of Pietsch (1969), are examples of the ways in which the tensile strength of a moist non-cohesive agglomerate (limestone) vary with the liquid saturation $\mathrm{S}$. The saturation is defined as the fraction of pore space taken up by liquid, as set forth in Equation (8.4).

$$
S=\frac{\varepsilon_{c}}{1-\varepsilon_{s}}
$$

where $\varepsilon_{\mathrm{c}} \quad=$ volume fraction of liquid

In the agglomerates represented by Figures 8.1 and 8.2 , the tensile strength between $S=0$ and $S=S_{b}$ depends on the forces exerted by the liquid bridges between the particles. The strength of these bridges results from a decreased (capillary) pressure within the liquid and from surface tension where the bridges meet the particles. At $S=S_{b}$, the bridges begin to contact each other and some pores begin to fill with liquid. As saturation increases, more pores fill until finally at $S=S_{c}$ all the pores are entirely filled and the agglomerate strength depends on the capillary pressure. In the absence of solid-solid forces, as in the limestone agglomerate, the tensile strength can be taken as going to zero at $S=1$, where the agglomerate is reduced to particles in liquid, and at dryness when $\mathrm{S}=0$.

During drainage, when liquid is displaced by gas, the effect of capillary pressure is different than during imbibition, when gas is displaced by liquid. In the absence of simultaneous mechanical deformation, imbibition cannot proceed to complete saturation; some pores are typically blocked to liquid flow. The result is that capillary pressure and tensile strength are lower during imbibition than during drainage, and drop to zero at a value $S_{a}<1$. For drainage, the zero point is at $S_{a}=1$. 
For cohesive agglomerates, such as clays and (probably) Hanford waste sludges, the yield behavior may be somewhat different because solid-solid forces are added to those contributed by the presence of liquid and gas. However, data for high-saturation compressed clay (Leavell and Peters 1987, Fig. 9) suggests that the overall behavior is similar. For these samples, the peak tensile strength at $S_{c}=0.84$ drops by a factor of 4 or more as the saturation decreases to $S=0.5$, and $S_{a}$ is about 0.90. The peak tensile strength varies with the pressure applied during clay sample compression, but $S_{c}$ does not so vary. No information about $S_{b}$ can be deduced from this data set.

A full description of the stress-strain behavior of an agglomerate requires not only the peak stress required to cause failure (the strength), but the strain at which failure occurs (that is, the ductility). Schubert et al. (1975) show the tensile stress for a moist agglomerate in the pendular regime does not depend linearly on strain. The functional form of the dependence is shown in Equation (8.5):

$$
\begin{aligned}
& \tau=\tau_{\mathrm{t}}\left[1-\left(1-\frac{\Delta \mathrm{L}}{\Delta \mathrm{L}_{\max }}\right)\right]^{3 / 2} \\
& \text { where } \tau=\text { tensile stress } \\
& \tau_{t} \quad=\text { tensile strength } \\
& \Delta \mathrm{L} \quad=\text { elongation } \\
& \Delta \mathrm{L}_{\max }=\text { maximum elongation at failure; } \mathrm{E} \text { is defined as } \Delta \mathrm{L}_{\max } / \mathrm{L} \text {, with } \mathrm{L} \text { being the } \\
& \text { length before strain was caused. }
\end{aligned}
$$

Thus, experimental data must give final elongation, as well as tensile strength.

Lacking a more complete model of stress-strain relations -- one which includes the capillary regime -- stress in the agglomerate could be calculated using the form of Equation (8.5), with an exponent derived from data correlation in place of the theoretical $3 / 2$ power. An exponent of 1.0 would give the simple linear stress-strain relation.

Data and models are more common for the tensile strength of agglomerates than for the shear strength. According to Schubert et al. (1975), no single relationship between the shear strength and the tensile strength applies for all agglomerate materials. However, it is possible the shear strength and tensile strength have the same scalings with regard to particle size, surface tension, and porosity. 
In the dendritic bubble regime, a submodel is needed that can fit the type of yield strength data that have been taken in the literature. The curve-fit model shown in Figures 8.1 and 8.2 (for the pendular, capillary, and total tensile strength) fills the requirements for the dendritic bubble regime, as can be seen from the close match between the curve fit for tensile strength and the data. So long as the shear strength is related to the tensile strength by a proportionality constant, it too should be describable by this model. The equations for these models follow as Equations (8.6) through (8.8):

\section{Pendular:}

$$
\begin{aligned}
& \tau_{s b, p}=\left(\frac{\varepsilon_{s}}{1-\varepsilon_{s}}\right) \frac{\sigma C_{1} S_{b}}{D_{s}}\left(\frac{S}{S_{b}^{2}+S^{2}}-\frac{S_{a}}{S_{b}^{2}+S_{a}^{2}}\right) \quad \text { for } S<S_{a} \\
& \tau_{s b, p}=0 \quad \text { for } S \geq S_{a}
\end{aligned}
$$

\section{Capillary:}

$$
\begin{array}{ll}
\tau_{s b, c}=\left(\frac{\varepsilon_{s}}{1-\varepsilon_{s}}\right) \frac{\sigma C_{2}}{D_{s}}\left(S-S_{b}\right)^{\alpha}\left(S_{a}-S\right)^{\alpha\left(S_{a}-S_{c}\right) / S_{a}} \quad \text { for } S_{b}<S<S_{a} \\
\tau_{s b, c}=0 \quad \text { for } S \leq S_{b}, S \geq S_{a}
\end{array}
$$

Total:

$$
\tau_{\mathrm{sb}}=\tau_{\mathrm{sb}, \mathrm{p}}+\tau_{\mathrm{sb}, \mathrm{c}}
$$




$$
\begin{aligned}
& \text { where } \begin{array}{ll}
\tau_{\mathrm{sb}, \mathrm{p}} & =\text { pendular contribution to yield strength } \\
\tau_{\mathrm{sb}, \mathrm{c}} & =\text { capillary contribution to yield strength } \\
\tau_{\mathrm{sb}} & =\text { total yield strength } \\
\sigma & =\text { surface tension } \\
\mathrm{D}_{\mathrm{s}} & =\text { particle diameter } \\
\mathrm{S} & =\text { saturation (fraction of non-solid volume occupied by liquid) } \\
\mathrm{S}_{\mathrm{a}} & =\text { saturation at which imbibition ends } \\
\mathrm{S}_{\mathrm{b}} & =\text { saturation at which peak pendular strength occurs } \\
\mathrm{S}_{\mathrm{c}} & =\text { saturation at which peak capillary strength occurs } \\
\alpha & =\text { fitting parameter for capillary pressure } \\
\mathrm{C}_{1} & =\text { fitting parameter for maximum capillary strength } \\
\mathrm{C}_{2} & =\text { fitting parameter for maximum pendular strength }
\end{array}
\end{aligned}
$$

All of the constants $\left(C_{1}, C_{2}, \alpha, S_{a}, S_{b}\right.$, and $\left.S_{c}\right)$ must be derived from experimental data. The scalings for surface tension, particle size, and porosity are taken from the literature (Schubert et al., 1975; Schubert, 1977). Nevertheless, it is not clear the scalings are appropriate. Figures 8.1 and 8.2 show an increase in the capillary strength as particle size decreases, which matches Equation (8.7), but a decrease in the pendular strength, which does not match Equation (8.6).

A similar disagreement with the theoretical particle-size scaling has appeared in other data. Gauglitz et al. (1994b) measured the shear yield strength for wetted packed beds of monodisperse glass beads that had diameters ranging from 10 to $500 \mu \mathrm{m}$. These experiments simulated the behavior of agglomerates containing dendritic bubbles. The dendritic bubble submodel, Equation (8.8), predicts a yield strength proportional to the quantity $\left(\sigma / \mathrm{D}_{\mathrm{s}}\right)$. However, the data of Gauglitz et al. (1994b) are better represented by a proportionality of the form $\left(\sigma / D_{s}\right)^{0.7}$. When the bead diameter was $30 \mu \mathrm{m}$ or less, the measured shear yield strength exhibited a strong maximum in the saturation range of 0.25 to 0.40 , matching the behavior of the pendular yield strength. The data also suggested the presence of a secondary maximum yield strength at saturation greater than 0.70 , possibly indicating a contribution from the capillary yield strength for small particle sizes $(30 \mu \mathrm{m}$ or less). 
A model is also needed for the yield strength of sludge in the spherical bubble regime. In this regime, the spherical bubbles are large enough to be seen and their effects are not related to the surface tension in the pores between particles. The following naive approach can be taken to find a simple model to describe the effects of bubbles on the macroscopic strength of the agglomerate.

In a shear plane that contains bubbles, the area fraction of the bubbles on the plane is roughly equal to the volume fraction of bubbles in the agglomerate, or $\varepsilon_{\mathrm{b}}$. To find the bulk shear strength of the agglomerate/bubble mixture, the resistance to shear deformation of both the solid and the bubbles must be taken into account. A detailed derivation of a model that includes both of these factors can be found in Gauglitz et al. (1994b). The end result is seen in Equation (8.3).

This model, though unsophisticated, suggests a form and scaling for the stress/strain relation in the spherical bubble regime. The ductility of the bubbly agglomerate must be determined experimentally.

One possible consequence of the difference in yield strength for dendritic and spherical bubbles is that an element of sludge that changes from one morphological regime to the other may also change dramatically in strength. Depending on the bubble size, the sludge could be considerably weaker, if it contained spherical bubbles, than if the bubbles were dendritic. Also note that sludge containing dendritic bubbles may grow stronger as void fraction increases, but sludge with spherical bubbles will grow weaker.

\subsubsection{Multi-component}

No data were found to support correlations to describe multi-component effects on the bubbly sludge yield strength. 


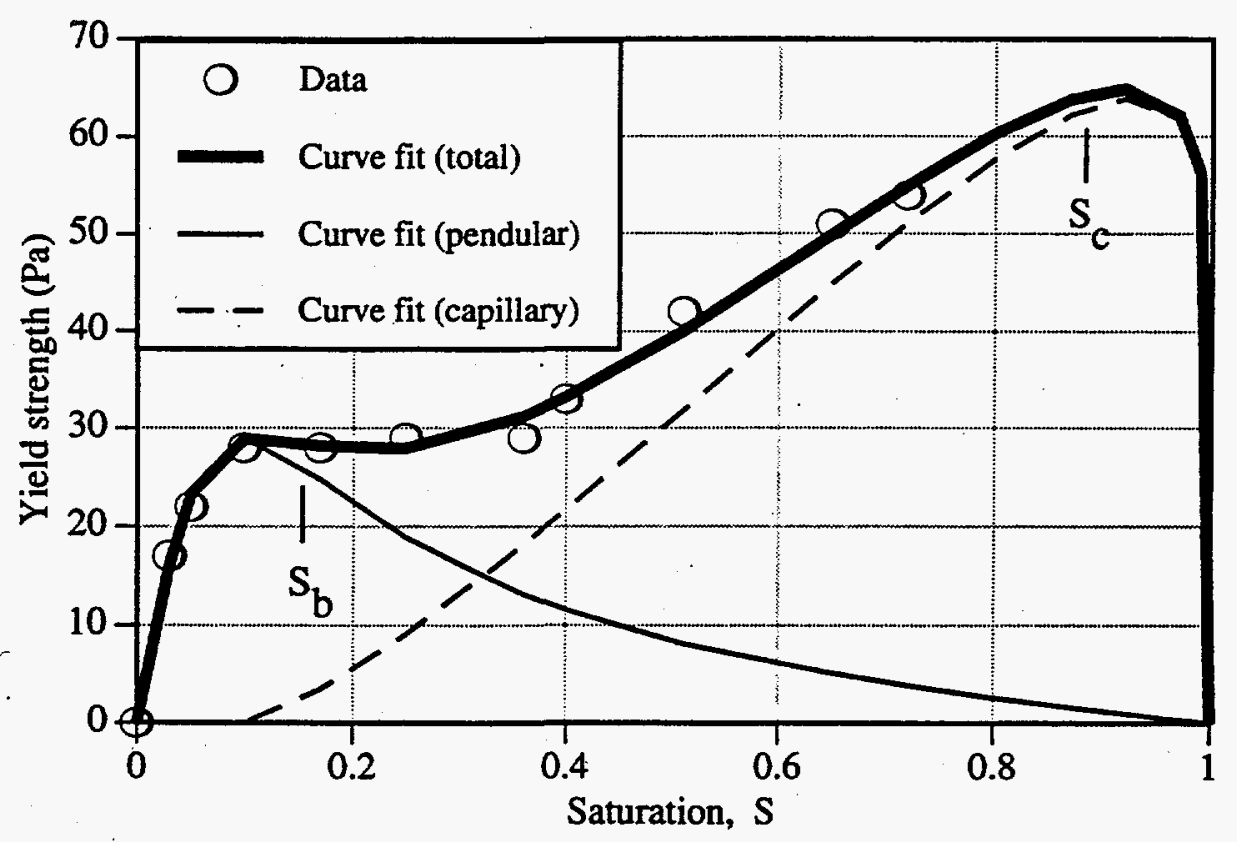

Figure 8.1. Tensile yield strength for a moist agglomerate of $71-\mu \mathrm{m}$ limestone particles.

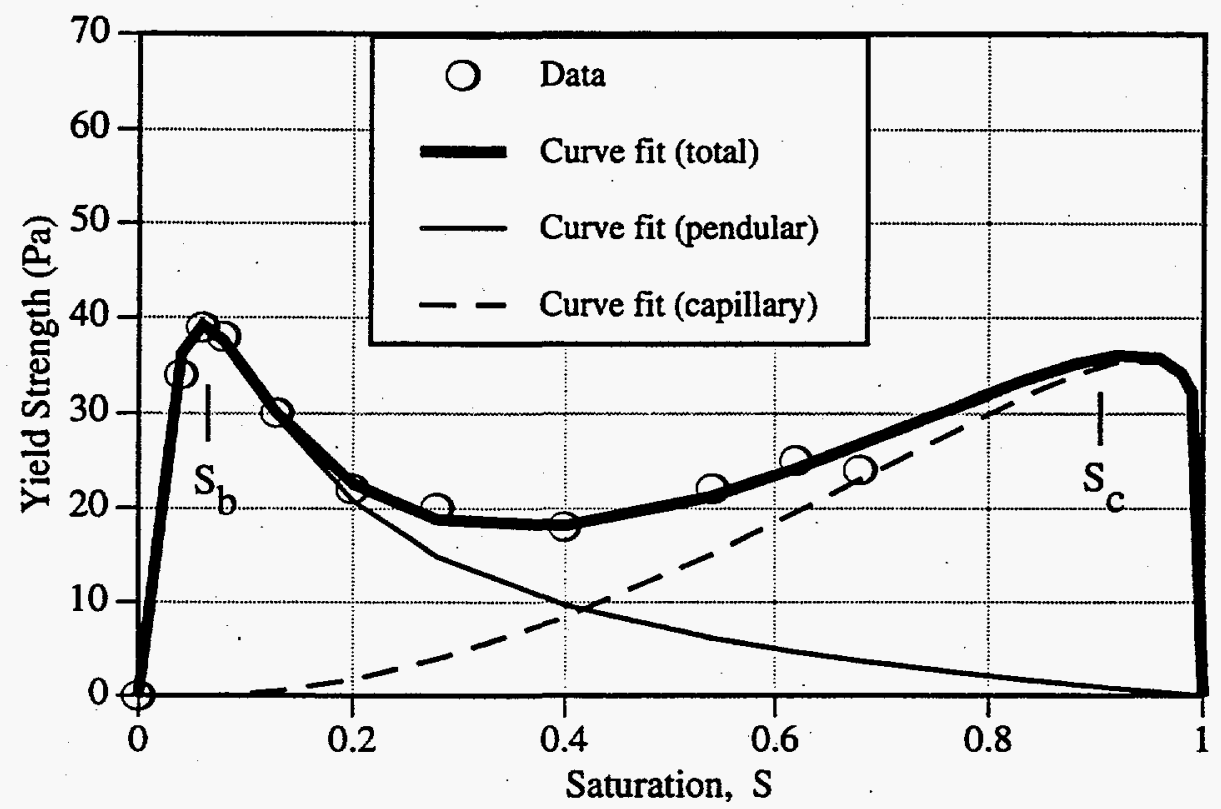

Figure 8.2. Tensile yield strength for a moist agglomerate of $185-\mu \mathrm{m}$ limestone particles. 


\subsection{Sludge Rheology}

After stresses in the sludge have exceeded its static yield strength (Section 8.0), the rheology of the fluid sludge and slurry in the Hanford waste tanks is a primary concern. The rheological behavior of both the non-Newtonian sludge and Newtonian supernatant significantly influences the flow of the fluid during a gas release event and during remediation and mitigation efforts.

The purpose of this section is to discuss many of the more common non-Newtonian rheological submodels for suspensions and demonstrate how well they do (or do not) match the rheological behavior of waste sludge. The data used for comparison to the submodels were obtained from two different data sets. The first set comprised data from the settled sludge layer in Tank 241-SY-101. The data were taken under several different conditions of dilution and temperature. The second data set contained stress-shear rate measurements of radioactive sludge samples (not simulants) from ORNL's Melton Valley Storage Tank facility; solids concentrations were lower for the ORNL sludge than for the SY-101 sludge. The SY-101 sludge data (described in Section 9.3.1) showed a linear relationship between stress and shear rate over most of the range of measurements, and were best modeled by the Bingham or biviscous models. The ORNL sludge data proved to be best modeled by the biviscous, Cross, or Carreau models; the Herschel-Bulkley and Casson models provided rather poor fits, as discussed in Section 9.3.2.

Section 9.1 summarizes the general types of rheological submodels for homogeneous and heterogeneous flow that are found in the literature. Section 9.2 describes the data and submodels for the clear liquid phase in the waste. Section 9.3 concerns the rheological behavior of the gas-free suspension, and Section 9.5 does the same for the bubbly sludge and slurry. Finally, Section 9:5 contains brief abstracts of a number of pertinent papers, focusing on the measurement methods and the models that were applied.

\subsection{General discussion}

The classic rheological description of a viscous fluid is given in Equation (9.1):

$$
\tau=-\eta \mathbf{D}
$$

In the Newtonian case, the shear stress tensor $\tau$ is a linear function of the shear rate tensor $\mathbf{D}$; the viscosity $\eta$ is not a function of $\mathbf{D}$ or of time but only of solids concentration, temperature and 
possibly other local variables. For a purely viscous non-Newtonian fluid, such as a typical sludge, the viscosity is a (frequently complicated) function of $\mathbf{D}$.

Some of the most desirable characteristics of rheological models include a minimal number of adjustable (experimentally obtained) constants; a form that allows easy curve-fitting to data; computational simplicity to maintain a reasonable computation time; and physical meaning. The following subsections discuss the purely viscous models in the literature with respect to these criteria. For convenience, the models are subdivided into two types, those with and those without yield stresses. Rheological models that include thixotropy (that is, time-dependent rheological behavior) are not discussed here, because data are not available to quantitatively support these more complex models.

The first broad set of purely viscous (time-independent) non-Newtonian rheological submodels comprises the models without shear yield stresses. In the no-yield models, the presumption is that every stress, no matter how small, leads to a continuing deformation. Fluids in which the viscosity decreases as the shear rate increases are known as shear-thinning or pseudoplastic.

The models without yield stresses have finite viscosity at very low shear rates; this is computationally desirable because of its bounded behavior, but it poses a computational inconvenience. In any computation where fluid shear stress is being calculated using a shear-rate algorithm, and the shear rate approaches zero; the numerical error in the computer model leads to calculation of infinitesimal shear stresses in systems where the true shear stress is zero. For finite viscosities, the shear stress error produces very small but non-zero shear rates, which over a prolonged period overpredict the fluid deformation.

The second set of non-Newtonian submodels are those that include a yield stress, which can be defined as a minimum shear stress below which no continuing deformation occurs. Below the yield stress, such a material behaves like a solid with a finite deformation; above it, behavior is fluid, with a finite shear rate and unbounded deformation. Fluids that display a yield stress are termed viscoplastic.

Some controversy appears in the literature about whether such a property as yield stress really exists or whether a fluid that appears to have one only has extremely high viscosity at very low shear rates (Ellwood et al.1990; Evans 1992; Husband et al. 1993; Kalyon et al. 1993). Part of the difficulty is that a very lengthy experiment may be required to tell the difference between a very slow 
continuing shear rate and a finite, final deformation. Further confusion comes from the distinction between constant-stress and constant-shear-rate viscometry (Husband et al. 1993), and from some experimenters' neglect of wall-slip measurements.

Constant-stress viscometry (such as a falling-ball device) measures a static yield stress, the minimum stress needed to cause unbounded shear (a steady-state shear rate). That is, the static yield stress is the yield strength of the solid. Constant-shear-rate viscometry measures a dynamic yield stress, the stress determined by extrapolating a stress-shear-rate plot to zero shear rate: The dynamic yield stress is in effect a fluid, not a solid, property. A material can easily have a static but no dynamic yield stress (Husband et al. 1993). The dynamic yield stress is the one rheological submodels typically use. [For a review of the current methods of measuring yield stress, refer to Nguyen and Boger (1992).]

Yield stresses can be strongly dependent on the recent shear history of the suspension. The first occurrence of a high shear rate (after a long quiescent period) may experimentally show a much higher yield stress than later tests display. This one-time yield stress can be the result of interparticle bonds that formed during the undisturbed period, or of normal stresses that result from the constrained configuration of the viscometer. Unconstrained mixtures would show different and more predictable behavior (Kytomaa 1992).

\subsection{Single-phase liquid rheological behavior}

Though liquids, as well as slurries and sludges, can exhibit non-Newtonian behavior, the supernatant liquid in the tanks has been determined to behave as a Newtonian fluid(9) and, therefore, is discussed as such.

\subsubsection{Single component}

Although the solution in the Hanford waste tanks contains numerous dissolved species, the sodium nitrate and nitrite components are so predominant as to suggest that a single-component submodel could be used. In such a model, one of the major aqueous species is used as a surrogate for all. A template for the dynamic viscosity of a single-component solution is given in Equation (9.2).

(9) Tingey, J.M. 1992. Rheological Properties of Waste from Tank 101-SY. Pacific Northwest Laboratory, Richland, Washington. May 1992. 


\subsubsection{Template for $\mu_{c}$, the liquid viscosity (Pa-s)}

$$
\mu_{c}=a_{4} \exp \left(\left[a_{0}+a_{1} C+a_{2} C^{2}\right] / T\right)
$$

Range: temperature and concentration as stated below

$$
\begin{aligned}
& \text { where } a_{k}=\text { coefficients obtained by data correlation } \\
& \text { C . = concentration of solute by weight percent of the total solution }
\end{aligned}
$$

\subsubsection{Data}

The data in Table II of Janz et al. (1970) and Table 5 of Zaltash and Ally (1992) allowed the correlation of $\mathrm{NaNO}_{3}$ solution viscosity in the form of Equation (9.2):

$$
\begin{aligned}
& \mathrm{a}_{4}=5.8044 \times 10^{-6} \mathrm{~Pa}-\mathrm{s} \\
& \mathrm{a}_{0}=1489.9 \\
& \mathrm{a}_{1}=-0.97874 \\
& \mathrm{a}_{2}=0.19490
\end{aligned}
$$

The correlation coefficient $\left(\mathrm{r}^{2}\right)$ of the correlation was 0.995 and the error in viscosity was $10 \%$ or less. The data were given for 5 different temperatures and concentrations over a concentration range from 0 to $90 \mathrm{wt} \% \mathrm{NaNO}_{3}$ and a temperature range from 273 to $433 \mathrm{~K}$.

Treating all the dissolved solids in solution as $\mathrm{NaNO}_{3}$, and using Equation (9.2), a concentration of $88 \mathrm{wt} \%$ dissolved $\mathrm{NaNO}_{3}$ (or other solutes for which it is the surrogate) would be required to produce the viscosity of about $0.06 \mathrm{~Pa}-\mathrm{S}$ at $32{ }^{\circ} \mathrm{C}$ that has been observed for $\mathrm{SY}-101$ convective-layer liquid(10). The actual concentrations of dissolved solids are 40 to $50 \mathrm{wt} \%$, for which concentration Equation (9.2) predicts a solution viscosity of about $0.003 \mathrm{~Pa}$-s.

The $\mathrm{NaNO}_{3}$ correlation can also be compared to the density and viscosity data for the supernatant in ORNL waste storage tank W-28 (Ceo et al. 1990). This supernatant had a density of $1290 \mathrm{~kg} / \mathrm{m}^{3}$ and a viscosity (at $21^{\circ} \mathrm{C}$ ) of $0.0022 \mathrm{~Pa}-\mathrm{s}$. According to the solution density correlation

(10) Tingey, J.M. 1992. Rheological Properties of Waste from Tank 101-SY. Letter report to Westinghouse Hanford Company, Pacific Northwest Laboratory, Richland, Washington. May 1992. 
in Table 4.2 of this report, the solution density corresponds to a solute concentration of about $40 \mathrm{wt} \%$. At this concentration, and at $21^{\circ} \mathrm{C}$, the $\mathrm{NaNO}_{3}$ viscosity correlation predicts a viscosity of $0.0023 \mathrm{~Pa}-\mathrm{s}$, very close to the measured value.

These comparisons suggest that some undissolved solids were present in the SY-101 convective layer and substantially increased the viscosity over clear-liquid values. In fact, some solids were present, as shown by the measurements of minimum $8 \mathrm{wt} \%$ centrifuged solids in the convective layer(11).

If the viscosity of the SY-101 convective layer liquid (undissolved solids and all) is correlated versus temperature, the result is

$$
\begin{aligned}
& a_{4}=1.226 \times 10^{-6} \mathrm{~Pa}-\mathrm{s} \\
& a_{0}=3218 .
\end{aligned}
$$

This correlation is accurate to within $23 \%$ within the range of 305 to $353 \mathrm{~K}$. Because the correlation includes some solids effect, it is not appropriate to use it as the viscosity of the suspending medium in estimates of the suspension viscosity. Section 9.3.1.3.1 contains a separate correlation for the gasfree waste suspension.

\subsubsection{Multi-component}

No data were found from which to derive a submodel for multi-component solution viscosity,

\subsection{Liquid-solid (gasless) slurry rheological behavior}

The slurries and sludges in Hanford waste tanks are known to exhibit shear-thinning nonNewtonian rheological traits(12). However, the non-Newtonian rheological submodels that best describe the waste behavior had not been identified by earlier work and were tested by correlation to tank waste data.

(11) Tingey, J.M. 1992. "Physical Characterization of Tank 101-SY Core Samples from Window C." Letter report to Westinghouse Hanford Company, Pacific Northwest Laboratory, Richland, Washington. February 1992.

(12) Tingey, J.M. 1992. Rheological Properties of Waste from Tank 101-SY. Pacific Northwest Laboratory, Richland, Washington. May 1992. 


\subsubsection{Single component}

Treating the slurries and sludges as a single-component suspension allows the use of correlations that depend not on chemical composition, but only on the relative amounts of solid and liquid. The waste slurries and sludges -- a slurry being a suspension with a concentration below the maximum packing fraction, and a sludge being a suspension that is fully packed -- are multicomponent systems. It is not clear whether minor components, such as the aluminates, can have major effects on the rheological behavior(13).

There are a number of possible submodels for the rheological behavior of a non-Newtonian suspension (or other fluid). These are given in Equations (9.3) through (9.9). Possible templates for the concentration dependence of the rheological parameters are given in Equations (9.11) through (9.14).

\subsubsection{Templates for shear rate dependence of $\tau$, the fluid shear stress (Pa)}

Power law submodel:

$$
\tau=-\left\{K\left|\sqrt{\frac{1}{2} \Pi_{D}}\right|^{n-1}\right\} D
$$

where $\mathbf{D} \quad=$ shear shear rate tensor

$\Pi_{D} \quad=$ second invariant of the shear rate tensor

$\mathrm{K} \quad=$ consistency index

$\mathrm{n}=$ structural (or behavioral) index

In the power law, or Ostwald-DeWaele, submodel (Bird et al. 1960) the fluid is shear-thinning for $\mathbf{n}<1$, which is usually the case for suspensions and sludges. For very concentrated suspensions, $\mathbf{n}$ may exceed 1 and the fluid is dilatant, becoming more viscous with increasing shear rate. The major computational difficulty with the power-law model is that (for $n<1$ ) the viscosity becomes unrealistically large at low shear rates.

(13) LaFemina, J.P., et al. 1995. Tank Waste Treatment Science: Report for the First Quarter FY 1995. TWRSPP-94-118. Letter report to Westinghouse Hanford Corporation. January 19, 1995. 
Cross (1965) submodel:

$$
\tau=-\left\{\eta_{\infty}+\frac{\left(\eta_{0}-\eta_{\infty}\right)}{1+B\left|\sqrt{\frac{1}{2} \Pi_{D}}\right|^{n}}\right\} \mathbf{D}
$$

$$
\begin{aligned}
& \text { where } \eta_{\infty} \quad=\text { Newtonian viscosity at high shear rate }(20-100 / \mathrm{s}) \\
& \begin{aligned}
\eta_{0} & =\text { Newtonian viscosity at very low shear rate }(0.01-0.5 / \mathrm{s}) \\
\mathrm{B} & =\text { correlating constant specific to the fluid }
\end{aligned}
\end{aligned}
$$

This model was based on the assumption that pseudoplastic behavior occurred in suspensions because of the formation and breakage of structural linkages between particles. A kinetic approach was used to estimate the average group size as a function of shear, and the viscosity was considered to be proportional to group size (assuming the groups are in the form of kinked chains). The Cross model (Cross 1965) gives a finite viscosity of $\eta_{0}$ as the shear rate approaches zero.

Carreau submodel:

$$
\tau=-\left\{\eta_{\infty}+\left(\eta_{0}-\eta_{\infty}\right)\left[1+\left(B\left|\sqrt{\frac{1}{2} \Pi_{D}}\right|\right)^{2}\right]^{(n-1) / 2}\right\} \mathbf{D}
$$

In this model, discussed by Ellwood et al. (1990), the parameter B is a characteristic relaxation time. The Carreau model gives a finite viscosity at low shear rates.

Biviscous submodel:

$$
\tau=-\left\{\eta_{\infty}+\frac{\left(\eta_{0}-\eta_{\infty}\right)}{B\left|\sqrt{\frac{1}{2} \Pi_{D}}\right|}\left(1-\exp \left(-B \sqrt{\frac{1}{2} \Pi_{D}}\right)\right)\right\} D
$$


Here $\mathrm{B}$, the biviscosity constant, controls the breadth of the region over which the viscosity changes from its low-shear-rate to its high-shear-rate value. This submodel describes a fluid that has two different finite Newtonian viscosities, one at very low and one at high shear rates (Kalyon et al. 1993).

Bingham submodel (Bird et al. 1960):

$$
\begin{aligned}
& \tau=-\left\{\eta_{\infty}+\frac{\tau_{0}}{\left|\sqrt{\frac{1}{2} \Pi_{D}}\right|}\right\} \text { D for } \frac{1}{2} \Pi_{\tau}>\tau_{0}^{2} \\
& \text { and } D=0 \text { for } \frac{1}{2} \Pi_{\tau}<\tau_{0}^{2}
\end{aligned}
$$

Casson submodel (Bird et al. 1983):

$$
\begin{aligned}
\tau=- & \left\{\sqrt{\frac{\tau_{0}}{\mid \sqrt{\frac{1}{2} \Pi_{D} \mid}}}+\sqrt{K}\right\}^{2} D \quad \text { for } \frac{1}{2} \Pi_{\tau}>\tau_{0}^{2}
\end{aligned}
$$

Herschel-Bulkley submodel (Bird et al. 1983):

$$
\tau=-\left\{K\left|\sqrt{\frac{1}{2} \Pi_{D}}\right|^{n-1}+\frac{\tau_{0}}{\sqrt{\frac{1}{2} \Pi_{D}}}\right\} D \quad \text { for } \frac{1}{2} \Pi_{\tau}>\tau_{0}^{2}
$$

$$
\text { and } \mathrm{D}=0 \text { for } \frac{1}{2} \Pi_{\tau}<\tau_{0}^{2}
$$


Gay submodel (Dabak and Yucel 1987):

$$
\begin{aligned}
\tau= & -\left\{\frac{\tau_{0}}{\mid \sqrt{\frac{1}{2} \Pi_{D}}}+\eta_{\infty}+\frac{\left(\eta_{0}-\eta_{\infty}\right)}{1+\frac{\left(\eta_{0}-\eta_{\infty}\right)\left|\sqrt{\frac{1}{2} \Pi_{D}}\right|}{B}}\right\} \text { D for } \frac{1}{2} \Pi_{\tau}>\tau_{0}^{2} \\
& \text { and } D=0 \text { for } \frac{1}{2} \Pi_{\tau}<\tau_{0}^{2}
\end{aligned}
$$

$$
\begin{aligned}
& \text { where } \begin{array}{ll}
\tau & =\text { shear stress tensor } \\
\Pi_{\tau} & =\text { second invariant of the shear stress tensor } \\
\mathbf{D} & =\text { shear rate tensor } \\
\Pi_{\mathrm{D}} & =\text { second invariant of the shear rate tensor } \\
\tau_{0} & =\text { yield stress } \\
\eta_{\infty} & =\text { Newtonian viscosity at high shear rate }(20-100 / \mathrm{s}) \\
\eta_{0} & =\text { Newtonian viscosity at very low shear rate }(0.01-0.5 / \mathrm{s}) \\
\mathrm{K} & =\text { consistency index } \\
\mathrm{n} & =\text { structural (or behavioral) index } \\
\mathrm{B} & =\text { correlation constant specific to the fluid }
\end{array}
\end{aligned}
$$

All of these submodels approach an infinite viscosity $\eta$ as $\mathbf{D}$ goes to zero (Bird et al. 1983) because $\Pi_{D}$ (which also goes to zero) is in the denominator of the shear stress terms. The yield-stress models also exhibit a discontinuity at $\mathbf{D}=0, \tau=\tau_{0}$. These computational problems can be avoided by substituting a stiff exponential for $\tau_{0}$ (Ellwood et al. 1990), as shown in Equation (9.10).

$$
\tau_{0}=\left|\tau_{0}\right|\left(1-\mathrm{e}^{-\mathrm{m}\left|\sqrt{\frac{1}{2} \Pi_{\mathrm{D}}}\right|}\right)
$$

Usually, $m$ is set for computational convenience rather than being determined from experimental data. However, data could also be used, providing a kind of biviscous model. The viscosity of the $m$-modified Bingham model (for example) approaches a value of $\left(\eta_{0} m+\eta_{\infty}\right)$ as 
$\Pi_{D}$ approaches zero, according to L'Hospital's Rule. Thus $\left(\eta_{0} m+\eta_{\infty}\right)$ can be taken as the value of viscosity at very low shear rates, or $m=\left(\eta_{0}-\eta_{\infty}\right) / \eta_{0}$. The m-modified Bingham model is the simplest (three-constant) form of a biviscous model, but the exponential model, Equation (9.10), could equally well be used in the more complicated Herschel-Bulkley, Casson, or Gay forms.

Of these models, the Herschel-Bulkley has the most general applicability; it and its subsets, the Bingham and power-law models, are the most commonly used correlations in the experimental literature. In addition, foams often behave as Bingham fluids (Okuzono et al. 1993). However, two references (Turian et al. 1992; Fam et al. 1989) stated the Casson or Bingham models fit the rheological data for their suspensions better than the Herschel-Bulkley model. Most viscoplastic materials exhibit time-dependent behavior (Mewis 1979, Berker and Van Arsdale 1992), but the effects of time-dependence usually are not well-established.

\subsubsection{Templates for solids concentration dependence of $\tau$, the fluid shear stress $(\mathrm{Pa})$}

$$
\begin{aligned}
& \xi=a_{0}+a_{1} C_{r}+a_{2}\left(C_{r}\right)^{2} \\
& \xi=\left(b_{0}+b_{1} C_{r}\right)\left(\left(C_{r}\right)^{b_{3}}+b_{4}\left(C_{r}\right)^{b_{5}}\right)
\end{aligned}
$$

Range: from 0 to about $95 \%$ of the maximum packing fraction (Probstein et al. 1994)

$$
\text { where } \xi \quad \begin{aligned}
= & \text { any property in a non-Newtonian rheological sub-model, including } \tau_{0}, \eta_{\infty}, \\
= & \eta_{0}, \mathrm{~K}, \mathrm{n}, \text { or } \mathrm{B} . \\
= & \text { coefficients obtained by correlation } \\
\mathrm{a}_{\mathrm{k}} \quad & \text { exponents obtained by correlation } \\
\mathrm{b}_{\mathrm{k}} \quad & \varepsilon_{\mathrm{s}} / \varepsilon_{\mathrm{s}, \mathrm{m}}=\text { the volume fraction of solid divided by the maximum packing } \\
& \text { fraction } \varepsilon_{\mathrm{s}, \mathrm{m}}
\end{aligned}
$$

The above equation forms are drawn from correlation and have been used to fit the available tank waste data; they are not derived from theory.

Theoretically-based expressions for the solids dependence of some rheological properties are available, but in general do not fit the tank waste data particularly well. Two such expressions for $\eta_{\infty}$ were presented by Probstein et al. (1994); these are given in Equations (9.13) and (9.14) for the sake of comparison. 
Sengun and Probstein:

$$
\eta_{\infty}=\eta_{c}\left\{1+a_{0}\left(\frac{C_{r}^{1 / 3}}{1-C_{r}^{1 / 3}}+2 C_{r}^{1 / 3}+\frac{1}{2} C_{r}^{2 / 3}+3 \ln \left(1-C_{r}^{1 / 3}\right)\right)\right\}
$$

Krieger-Dougherty:

$$
\eta_{\infty}=\eta_{c}\left(1-c_{r}\right)^{-a_{0} c_{r}}
$$

- Colloidal fine particles are the primary cause of the non-Newtonian behavior of a suspension. The varying balance between viscous forces, surface forces, and Brownian motion leads to the shearrate dependence of the suspension. Where viscous forces predominate (for large particles, high shear rate, or a high liquid viscosity $\mu_{c}$ ), the suspension viscosity becomes independent of the applied shear rate. Probstein et al. (1994), and other researchers, have had some success with modeling polydisperse suspensions by separating the effects of the fines from those of the coarse particle fraction. The coarse particles (those larger than about $3 \mu \mathrm{m}$, in the work cited) contributed no shear dependence, but rather multiplied the shear-dependent viscosity of the fines by a constant factor.

\subsubsection{Data}

Three sources of data on radioactive waste sludge rheology were reviewed to find data suitable for determining which rheological submodels best described waste sludges. One (Fow et al. 1986) described measurements made on a non-radioactive simulant of neutralized current acid waste (NCAW) from the double-shell tanks (DSTs). The measurements were made at ambient and elevated temperatures, and at two dilutions, and were intended to support design of a pump-pipe network to transport NCAW slurries. Although the sludge is of a relevant type, these data are incomplete for present purposes because the lowest shear rate used in measurements was $50 \mathrm{sec}^{-1}$. (However, this range of shear rates was an appropriate lower end for the design purpose of the reference.) More information about this data set and the rheological findings is given in Section 9.5.

A more immediately useful data set was found in Ceo et al. (1990). Samples were taken from typical radioactive sludges and supernatant liquids in the ORNL Melton Valley Storage Tanks (MVST). The sludge samples were taken in cores up to 20 inches deep. All the samples, supernatant, sludge, and sludge diluted with supernatant (from the same tank), were assayed for viscosity, using a 
Brookfield rotational viscometer. The sludge rheological measurements were made on samples that had been handled as little as possible and that had not been sonicated to assure mixing. All measurements were made at $21^{\circ} \mathrm{C}$, over a shear rate range of 0.08 to $17 \mathrm{sec}^{-1}$.

The third, and most appropriate, data set came from measurements of undiluted and diluted sludge from Tank 241-SY-101. The SY-101 sludge core samples were taken from the upper $0.2 \mathrm{~m}$ of the non-convective slurry layer during Window C, after the May 1991 gas release event. The sludge samples are believed to have been free of gas at the time they were studied. The core samples were mixed to form a composite sample upon which viscometric measurements were made. The composite sample was split into subsamples that were diluted with $2 \mathrm{M} \mathrm{NaOH}$ and held at temperatures of 50,70 , or $90^{\circ} \mathrm{C}$ during measurement. In each of these subsamples, the measurements included the volume and weight percent of settled solids, shear strength, yield point, rheological behavior, weight percent solids, and the densities of the sample and its constituent parts. A constant-temperature viscometer with concentric-cylinder geometry was used to make the viscosity measurements, which covered a shear rate range of 2 to $500 \mathrm{sec}^{-1}$. These tests are described in Tingey et al. (1994) and other sources(14).

The most complete data set from Ceo et al. (1990) was that for the Tank W-28 sludge. Tank W-28 at MVST contains predominantly low-level waste (LLW) concentrates. The sludge is mostly calcium, magnesium and aluminum hydroxides and carbonates; the supernatant liquid is a basic aqueous solution of sodium nitrate and fission products(15). The supernatant from Tank W-28 was Newtonian, but the sludge exhibited substantial non-Newtonian behavior. Some evidence of timedependent behavior was also present, but the data were insufficient to allow an assessment of thixotropy.

For W-28 sludge, the densities of the bulk and interstitial liquid were the same, $1290 \mathrm{~kg} / \mathrm{m}^{3}$. The density of the (centrifuged) bulk sludge was $1400 \mathrm{~kg} / \mathrm{m}^{3}$ and the undissolved solids density was $2000 \mathrm{~kg} / \mathrm{m}^{3}$. The bulk sludge contained $51.4 \mathrm{wt} \%$ total solids; these were subdivided into $29.4 \mathrm{wt} \%$ dissolved solids in the interstitial liquid and $22.0 \mathrm{wt} \%(15 \mathrm{vol} \%)$ undissolved solids. The supernatant liquid viscosity (at $21^{\circ} \mathrm{C}$ ) was $0.0022 \mathrm{~Pa}$-s. Based on the sedimentation rate of $2.9 \mathrm{~cm} / \mathrm{min}$, the W-28

(14) Bredt, P.R. 1994. "101-SY Dilution Studies." Letter to C.W. Stewart, Pacific Northwest Laboratory, Richland, Washington. July 26, 1994.

(15) Youngblood, E.L., J.B. Berry, and J.R. DeVore. 1991. "Predicting Transport Behavior for Radioactive Sludges at Oak Ridge National Laboratory." For presentation at the August 18-21, 1991 AIChE Summer National Meeting, Pittsburgh, Pennsylvania. 
sludge particle agglomerates were estimated to have an average radius of $26 \mu \mathrm{m}$ (Ceo et al. 1990). All the sludges studied appeared gas-free.

The Tank SY-101 sludge originated in a variety of processes used at Hanford. It consists chiefly of sodium and potassium nitrates, nitrites, and aluminates in a saturated, highly basic solution. Strontium and cesium compounds are also present. Viscometric measurements have shown the sludge has non-Newtonian rheological behavior, although the supernatant is a Newtonian fluid.

The viscosity of the Tank SY-101 supernatant, possibly including a small amount of solids, is $0.024 \mathrm{~Pa}$-s (at $50^{\circ} \mathrm{C}$ )(16). The supernatant density is $1450 \mathrm{~kg} / \mathrm{m}^{3}$, the bulk density of the gas-free sludge is $1670 \mathrm{~kg} / \mathrm{m}^{3}$, and the intrinsic solid density is $2100 \mathrm{~kg} / \mathrm{m}^{3}$. The sludge composition (in the absence of gas) is $42 \mathrm{wt} \%$ undissolved solids. The maximum gas content is 19 to $24 \mathrm{vol} \%$. Both the sludge and the supernatant for Tank SY-101 are significantly more concentrated than their MVST W-28 counterparts.

\subsection{Rheological submodels for the SY-101 sludge}

The sludge composite sample from Tank SY-101 was tested at several different dilutions: $0 \%$ dilution, $10 \%, 20 \%, 35 \%$, and $50 \%$. (These dilution percentages indicate the volume fraction of diluent in the final mixture, assuming that the sludge and diluent volumes are additive.) Each of these dilutions underwent measurements at three temperatures: 50,70 , and $90^{\circ} \mathrm{C}$. Typically, more than one measurement run was made with a sample at each dilution and temperature; the shear rate was first increased, then decreased, while the temperature was held constant and shear stress was measured. The runs have sequential codes: for example, runs AR2 and AR3 (in Figure 9.1) were made in that order. Experimental procedure was such that on each successive replicate run the sample had a higher cumulative shear history, but the exact shear history is unknown.

The solids concentration in the sludge at different dilutions depended on the specific diluent chemistry, not just the amount of dilution, because the $2 \mathrm{M} \mathrm{NaOH}$ that was used for dilution dissolved part of the original sludge solids. Figure 5.7 in Stewart et al. (1994) shows the solids concentrations (in weight percent) that have been estimated at different dilutions at $50{ }^{\circ} \mathrm{C}$. These are listed in

(16) Bredt, P.R. 1994. "101-SY Dilution Studies." Letter to C.W. Stewart, Pacific Northwest Laboratory, Richland, Washington. July 26, 1994.

Tingey, J.M. 1992. "Physical Characterization of Tank 101-SY Core Samples from Window C." Letter report to Westinghouse Hanford Company, Pacific Northwest Laboratory, Richland, Washington. February 1992. 
Table 9.1. It is not clear whether the apparent increase in solids with dilution, between $10 \%$ and $20 \%$ dilution, is a measurement artifact or the result of re-precipitation. The volume fractions of solids in Table 9.1 were calculated from the weight percents, using an average intrinsic solids density of 2200 $\mathrm{kg} / \mathrm{m}^{3}$ and measured liquid densities.

Table 9.1. Solids Fraction Versus Tank Dilution Ratio at $50^{\circ} \mathrm{C}$.

\begin{tabular}{clc} 
Dilution (\%) & Solids (wt\%) & Solids (vol\%) \\
\cline { 2 - 2 } & 42 & 32 \\
10 & 31 & 22 \\
20 & 39 & 29 \\
35 & 21 & 14 \\
50 & 10 & 6
\end{tabular}

Figures 9.1 through 9.15 show the experimental data points as symbols. The figures show that in most cases the sludge viscosity increases with increasing shear history (that is, with increasing cumulative shear). This gradual increase is particularly evident at low dilutions, for example at $10 \%$ dilution and $70^{\circ} \mathrm{C}$, as shown in Figure 9.7 . The increase of viscosity with cumulative shear has been observed in other sludges. One example is kaolin/water sludge, whose viscosity in a fully-sheared condition can be higher by a factor of 10 than the viscosity of unsheared sludge of the same composition (Selby 1981). After the kaolin/water sludge has undergone enough total shear, the viscosity levels off at a final maximum value. The data for the SY-101 sludge suggest some similar leveling-off; this can be surmised, for example, from Figure 9.2, where the latter two runs (V22 and V23) exhibit the same rheological behavior. For the higher-temperature runs, the gradual increase in viscosity may also be caused by the sample drying out and thickening.

Some degree of thixotropy can also be deduced from the data. Figure 9.2, among others, shows an initial sharp spike in shear stress in the first run (V21) during the increasing-shear-rate leg of the test. On the decreasing-shear-rate leg, which gives a near-linear stress-shear-rate relation, the shear stress is lower. In other cases (such as run V41 in Figure 9.3), the increasing leg gives a nearlinear relation while the decreasing leg shows lower stress and a nonlinear stress/shear rate relation for the same shear rates. Almost always, the increasing leg, which is the first step in the measurement procedure, gives the higher and less linear shear stresses.

Because of the variation in shear stress from one run to another, or from one leg of a run to another, the correlation effort was generally confined to the runs/legs that gave the highest (and 
putatively final) viscosity values. The initial spikes (probably representing a one-time yield strength) were ignored. The correlation includes only the range of the data for which the shear rate was 0 to $50 \mathrm{sec}^{-1}$ (the range of interest in the Hanford tank applications to be modeled by TEMPEST).

Because of the evident linearity of the stress-shear rate relation at high shear rates, only the Bingham and biviscous models were tested against the data. The difference in quality of fit between the Bingham and biviscous models was insignificant, probably the result of the very sparse amount of data taken at low shear rates (below $10 \mathrm{sec}^{-1}$ ). A more complete data set would have provided a stronger basis for correlation by the biviscous model (or any other no-yield model).

The curve-fit constants for the Bingham and biviscous rheological models are tabulated in Tables 9.2 through 9.6. All viscosities are expressed in units of (Pa-s) and all stresses in $\mathrm{Pa}$ (where $1 \mathrm{~Pa}=10$ dyne $/ \mathrm{cm}^{2}$ and $1 \mathrm{~Pa}-\mathrm{s}=1000 \mathrm{cP}$ ). Note that because of the small number of data points below a shear rate of $10 \mathrm{sec}-1$, the biviscous model values for $\eta_{0}$ and $B$ (as defined in Equation 9.6) should not be considered strongly based.

Table 9.2. Correlated yield stress $\tau_{0}(\mathrm{~Pa})$ for $S Y-101$ sludge fitted with a Bingham model.

\begin{tabular}{|c|c|c|c|}
\hline Dilution (\%) & $\tau_{0}$ at $50^{\circ} \mathrm{C}$ & $\tau_{0}$ at $70^{\circ} \mathrm{C}$ & $\tau_{0}$ at $90^{\circ} \mathrm{C}$ \\
\hline 0 & 3.60 & 4.90 & 8.74 \\
\hline 10 & 2.94 & 3.53 & 2.64 \\
\hline 20 & 1.07 & 2.07 & 2.33 \\
\hline 35 & 0.579 & 0.801 & 0.858 \\
\hline 50 & 0.568 & 0.471 & 0.582 \\
\hline
\end{tabular}

Table 9.3. Correlated viscosity $\eta_{\infty}(\mathrm{Pa}-\mathrm{s})$ for SY-101 sludge fitted with a Bingham model.

\begin{tabular}{|c|c|c|c|}
\hline Dilution (\%) & $\eta_{\infty}$ at $50^{\circ} \mathrm{C}$ & $\eta_{\infty}$ at $70^{\circ} \mathrm{C}$ & $\eta_{\infty}$ at $90^{\circ} \mathrm{C}$ \\
\hline 0 & 0.133 & 0.0924 & 0.0833 \\
\hline 10 & 0.0988 & 0.0621 & 0.0331 \\
\hline 20 & 0.0351 & 0.0396 & 0.0301 \\
\hline 35 & 0.0268 & 0.0166 & 0.00920 \\
\hline 50 & 0.00969 & 0.00948 & 0.00462 \\
\hline
\end{tabular}


Table 9.4. Correlated low-shear-rate viscosity $\eta_{0}(\mathrm{~Pa}-\mathrm{s})$ for $\mathrm{SY}$-101 sludge fitted with a biviscous model.

\begin{tabular}{|c|c|c|c|}
\hline Dilution (\%) & $\eta_{0}$ at $50^{\circ} \mathrm{C}$ & $\eta_{0}$ at $70^{\circ} \mathrm{C}$ & $\eta_{0}$ at $90^{\circ} \mathrm{C}$ \\
\hline 0 & 3.80 & 6.68 & 11.1 \\
\hline 10 & 2.38 & 2.64 & 85.0 \\
\hline 20 & 1.88 & 1.63 & 2.75 \\
\hline 35 & 0.540 & 0.736 & 0.752 \\
\hline 50 & 0.796 & 1.04 & 0.859 \\
\hline
\end{tabular}

Table 9.5. Correlated high-shear-rate viscosity $\eta_{\infty}(\mathrm{Pa}-\mathrm{s})$ for SY-101 sludge fitted with a biviscous model.

\begin{tabular}{llll} 
Dilution (\%) & $\eta_{\infty}$ at $50^{\circ} \mathrm{C}$ & $\eta_{\infty}$ at $70^{\circ} \mathrm{C}$ & $\eta_{\infty}$ at $90^{\circ} \mathrm{C}$ \\
\hline \begin{tabular}{lll}
0 \\
\hline 0
\end{tabular} & 0.125 & 0.0874 & 0.0719 \\
10 & 0.0905 & 0.0491 & 0.0331 \\
20 & 0.0346 & 0.0339 & 0.0271 \\
35 & 0.0256 & 0.0146 & 0.00704 \\
50 & 0.00923 & 0.00940 & 0.00405
\end{tabular}

Table 9.6. Correlated biviscosity constant B (sec) for SY-101 sludge fitted with a biviscous model.

\begin{tabular}{|c|c|c|c|}
\hline Dilution (\%) & B at $50^{\circ} \mathrm{C}$ & $\mathrm{B}$ at $70^{\circ} \mathrm{C}$ & $\mathrm{B}$ at $90^{\circ} \mathrm{C}$ \\
\hline 0 & 0.951 & 1.30 & 1.20 \\
\hline 10 & 0.708 & 0.648 & 32.2 \\
\hline 20 & 1.69 & 0.700 & 1.12 \\
\hline 35 & 0.829 & 0.826 & 0.799 \\
\hline 50 & 1.35 & 2.17 & 1.42 \\
\hline
\end{tabular}


Further correlation is required because the TEMPEST model uses input that describes the rheological model parameters as functions of solids concentration. The Bingham model correlation constants in Tables 9.2 and 9.3, and the biviscous model correlation constants in Tables 9.4 through 9.6, can be treated as functions of the solids volume fraction. Fitting the correlation constants as functions of concentration, by using the undiluted sludge solids concentration of 0.32 as the maximum packing fraction $\varepsilon_{s, m}$, gives the following equations:

The Bingham model at $50^{\circ} \mathrm{C}$ :

$$
\begin{aligned}
& \tau_{0}=1.17\left(\mathrm{C}_{\mathrm{r}}+2.19\left(\mathrm{C}_{\mathrm{r}}\right)^{6.13}\right) \\
& \eta_{\infty}=0.0155\left(1+7.85\left(\mathrm{C}_{\mathrm{r}}\right)^{5.57}\right)
\end{aligned}
$$

The Bingham model at $70^{\circ} \mathrm{C}$ :

$\tau_{0}=1.74\left(\mathrm{C}_{\mathrm{r}}+1.85\left(\mathrm{C}_{\mathrm{r}}\right)^{5.81}\right)$

$\eta_{\infty}=0.0118\left(1+6.83\left(C_{r}\right)^{4.59}\right)$

The Bingham model at $90^{\circ} \mathrm{C}$ :

$\tau_{0}=2.69\left(C_{r}+2.25\left(C_{t}\right)^{31.1}\right)$

$\eta_{\infty}=0.00835\left(1+8.76\left(C_{r}\right)^{7.76}\right)$

The biviscous model at $50^{\circ} \mathrm{C}$ :

$$
\begin{aligned}
& \eta_{0}=\left(0.0151+1.78 C_{r}\right)\left(1+1.10\left(C_{r}\right)^{7.10}\right) \\
& \eta_{\infty}=0.0151\left(1+7.53\left(C_{r}\right)^{5.62}\right) \\
& B=1.21+0.0606 C_{r}-0.270\left(C_{r}\right)^{2}
\end{aligned}
$$

The biviscous model at $70^{\circ} \mathrm{C}$ :

$$
\begin{aligned}
& \eta_{0}=\left(0.0119+2.00 C_{r}\right)\left(1+2.32\left(C_{r}\right)^{15.8}\right) \\
& \eta_{\infty}=0.0119\left(1+6.25\left(C_{r}\right)^{5.98}\right) \\
& B=3.78-9.88 C_{r}+7.34\left(C_{r}\right)^{2}
\end{aligned}
$$


The biviscous model at $90^{\circ} \mathrm{C}$ :

$$
\begin{aligned}
& \eta_{0}=\left(0.00614+2.14 C_{r}\right)\left(1+4.18\left(C_{r}\right)^{8.68}\right) \\
& \eta_{\infty}=0.00614\left(1+10.4\left(C_{r}\right)^{6.43}\right) \\
& B=1.92-3.45 C_{r}+2.82\left(C_{r}\right)^{2}
\end{aligned}
$$

Figures 9.1 through 9.15 show the rheological data that were obtained and the curve fits expressed in Equations (9.15) through (9.29), where $C_{r}$ is the local solids volume fraction divided by the volume fraction at maximum packing. Insets show the behavior of the curve fits and the data as the shear rate approaches zero. The correlations can be seen in the figures, with solid lines representing Bingham model fits and dashed lines representing biviscous model fits.

Note the high-shear viscosity values at $\mathrm{C}_{\mathrm{r}}=0$ are consistently less than the viscosities measured for the SY-101 supernatant at the same temperatures, and are within a factor of two of the supernatant viscosities. This behavior is consistent with the hypothesis that the supernatant contained solids that affected its viscosity.

\subsection{Rheological submodels for the ORNL W-28 sludge}

The ORNL W-28 sludge data from Ceo et al. (1990) were analyzed to find which of the models listed in Section 9.1 best described the W-28 sludge rheology. For the W-28 data, the 0\% dilution corresponds to a solids concentration of about $15 \mathrm{vol} \%$, and the $50 \%$ and $75 \%$ dilutions therefore contain 7.5 vol\% and 3.8 vol\% solids. (Because the sludge is diluted with its own supernatant, no dissolution of solids results from dilution.) Figures 9.16 through 9.22 show the curve fits that were obtained for the several models.

The two-constant models, the power-law and Casson models, do not well represent the knee of the stress-shear rate data (Figures 9.16 and 9.21). Of the two, the power-law model provides a slightly closer fit. The Herschel-Bulkley model (Figure 9.20), a three-constant model, comes much closer to the $50 \%$ and $75 \%$ dilution data than does its subset the power-law model, but it needs a physically dubious negative yield-stress to do so. The other three-constant model, the biviscous model (Figure 9.19), is clearly well adapted to modeling the sludge at all dilutions.

The remaining models -- the Cross, Carreau, and Gay models -- are all four-constant models that include two different constant viscosities for low and high shear rates. The Gay model (Figure 9.22) includes a yield stress and fits the data slightly less well than the yieldless four-constant models. 
To all appearances, the Cross and Carreau models (Figures 9.17 and 9.18) provide equally good fits that are very close to the data.

A little more insight about model qualifications can be gained by looking at the curve-fit constants for the models. These constants are given in Table 9.7. In all cases, the units of shear stress are $\mathrm{Pa}$ and the units of shear rate are sec- $^{-1}$, so viscosities are in ( $\left.\mathrm{Pa}-\mathrm{s}\right)$ and yield stresses in $\mathrm{Pa}$ (where 1 $\mathrm{Pa}=10$ dyne $/ \mathrm{cm}^{2}$ and $1 \mathrm{~Pa}-\mathrm{s}=1000 \mathrm{cP}$ ).

Table 9.7. Correlation parameters for rheological models fit to the ORNL MVST W-28 stress/shear rate data.

Power-law [Equation (9.3)]:

$\begin{array}{llll}\text { W28 -- } & \mathrm{K}=10.2 ; & \mathrm{n}=0.666 ; & \mathrm{r}^{2}=0.97 \\ \text { W28 50\% -- } & \mathrm{K}=3.54 ; & \mathrm{n}=0.355 ; & \mathrm{r}^{2}=0.91 \\ \text { W28 75\% -- } & \mathrm{K}=1.37 ; & \mathrm{n}=0.295 ; & \mathrm{r}^{2}=0.91\end{array}$

Cross [Equation (9.4)]:

W28 - $\quad \eta_{0}=497 ; \quad \eta_{\infty}=8.18 ; \quad \mathrm{B}=370 ; \quad \mathrm{n}=1.29 ; \quad \mathrm{r}^{2}=1.00$

W28 50\% - $\quad \eta_{0}=4.95 ; \quad \eta_{\infty}=0.186 ; \quad \mathrm{B}=0.316 ; \quad \mathrm{n}=1.33 ; \quad \mathrm{r}^{2}=1.00$

W28 75\% -- $\quad \eta_{0}=3.58 ; \quad \eta_{\infty}=0.0526 ; \quad \mathrm{B}=1.29 ; \quad \mathrm{n}=1.093 ; \quad \mathrm{r}^{2}=0.99$

Carreau [Equation (9.5)]:

W28 -- $\quad \eta_{0}=20 ; \quad \eta_{\infty}=4.5 ; \quad \mathrm{B}=2.6 ; \quad \mathrm{n}=0.1$

W28 50\% -- $\quad \eta_{0}=4.56 ; \quad \eta_{\infty}=0.0339 ; \quad B=0.659 ; \quad n=0.0716 ; \quad r^{2}=1.00$

W28 75\% -- $\quad \eta_{0}=3.05 ; \quad \eta_{\infty}=.00207 ; \quad \mathrm{B}=1.87 ; \quad \mathrm{n}=0.164 ; \quad \mathrm{r}^{2}=0.99$ 
Biviscous [Equation (9.6)]:

W28 -- $\quad \eta_{0}=1220 ; \quad \eta_{\infty}=7.39 ; \quad B=544 ; \quad-r^{2}=1.00$

W28 50\% -- $\quad \eta_{0}=5.31 ; \quad \eta_{\infty}=0.0904 ; \quad B=0.729 ; \quad r^{2}=1.00$

W28 75\% -- $\quad \eta_{0}=3.30 ; \quad \eta_{\infty}=0.0548 ; \quad B=1.60 ; \quad r^{2}=0.99$

Herschel-Bulkley [Equation (9.9)]:

W28 - $\quad \tau_{0}=2.76 ; \quad \mathrm{K}=6.58 ; \quad \mathrm{n}=1.17 ; \quad \mathrm{r}^{2}=1.00$

$\mathrm{W} 2850 \%-\quad \tau_{0}=-258 ; \quad \mathrm{K}=261 ; \quad \mathrm{n}=.00656 ; \quad \mathrm{r}^{2}=0.97$

W28 75\% - $\quad \tau_{0}=-86.2 ; \quad \mathrm{K}=87.7 ; \quad \mathrm{n}=.00596 ; \quad \mathrm{r}^{2}=0.98$

Casson [Equation (9.8)]:

W28 -- $\quad \tau_{0}=0.854 ; \quad K=4.94 ; \quad r^{2}=0.99$

W28 50\% -- $\quad \tau_{0}=1.84 ; \quad \mathrm{K}=0.196 ; \quad \mathrm{r}^{2}=0.78$

W28 75\% -- $\quad \tau_{0}=0.806 ; \quad K=0.0497 ; \quad r^{2}=0.76$

Gay [Equation (9.10)]:

W28 - $\quad \tau_{0}=2.25 ; \quad \eta_{0}=7.39 ; \quad \eta_{\infty}=7.39 ; \quad B=1 ; \quad r^{2}=1.00$

W28 50\% -- $\quad \tau_{0}=-0.219 ; \quad \eta_{0}=6.94 ; \quad \eta_{\infty}=-0.018 ; \quad B=9.93 ; \quad r^{2}=1.00$

W28 75\% -- $\quad \tau_{0}=-0.542 ; \quad \eta_{0}=8.65 ; \quad \eta_{\infty}=0.0349 ; \quad \mathrm{B}=2.92 ; \quad \mathrm{r}^{2}=0.99$

The correlation coefficients ( $\mathrm{r}^{2}$ values) are consistently near unity for almost all models, indicating good fit. However, all of the yield-stress models require negative yield stresses $\tau_{0}$ to provide accurate stress predictions at low shear rates for the whole range of concentrations. The physical meaning of this correlation feature is unclear, and in general the yield-stress models do not seem the best choices for modeling this sludge. 
Of the models, the Cross, Carreau, and biviscous models are clearly the most appropriate. The Cross and biviscous models seem slightly preferable because the viscosity at high shear rates is always greater than the $0.0022 \mathrm{~Pa}$-s value found for the supernatant liquid. The Carreau model, on the other hand, predicts an $\eta_{\infty}$ of $0.0021 \mathrm{~Pa}-\mathrm{s}$ for the $75 \%$ diluted sludge. This slight incongruity could cause some inconvenience in computational modeling.

In addition, the low value of Carreau infinite-shear rate viscosity leads to implausibly low shear stresses at high shear rates. For example, at $100 \mathrm{sec}^{-1}$ the predicted shear stress for $75 \%$-diluted W28 sludge is 7.0 Pa for the Cross model and 4.0 Pa for the Carreau model. At $1000 \mathrm{sec}^{-1}$, for the same dilution, the shear stress is calculated at $54 \mathrm{~Pa}$ for the Cross model and $7.7 \mathrm{~Pa}$ for the Carreau. The Carreau model plateaus (for these particular sludges) and the Cross model does not.

One danger of using the Cross model is that the equation can, with certain parameters, predict a shear stress that reaches a maximum as shear rate increases and then decreases at higher shear rates. In the case of the W28 data, no such maximum is found between 0.01 and $1000 \mathrm{sec}^{-1}$ and this problem is not a concern. This question does not arise with the biviscous model, where the relation between stress and shear rate is unambiguously monotonic. Furthermore, the biviscous model requires only three constants and the Cross model needs four.

The biviscous model (Equation 9.6) appears to be best suited for modeling the MVST Tank W28 sludge rheology in the low shear-rate range where viscometric data were obtained. This indication may provide a clue as to the best choice of models for the Hanford tank sludges at low solids concentrations and low shear rates (those below $1 \mathrm{sec}^{-1}$ ). However, the higher solids content of the Tank 241-SY-101 sludges (42 vol\%) may require a yield-stress model where the W-28 sludge (at 15 vol\% solids) did not. The literature summarized in Section 9.5 suggests that yield stresses often appear in the 20 to 30 vol\% solids concentration range.

\subsubsection{Multi-component}

Information is insufficient to predict the effect of the chemical interactions in a multicomponent suspension on the suspension viscosity. However, the multi-component nature of the Hanford tank waste probably has significant effects. An ongoing study that is part of the TWRS Pretreatment Project has found the viscosity of boehmite and gibbsite suspensions depends on the extent to which the particles agglomerate. This, in turn, depends on the $\mathrm{pH}$, the ionic strength, and the organics content of the solution. Variations of viscosity between 0.005 and $10 \mathrm{~Pa}$-s have been 
observed as the chemical composition of the solution is changed. The composition of the solid phase is also important, because it governs the surface charge of the particles and, as a result, the electrostatic forces that act on the particles and control agglomeration(17).

\subsection{Liquid-solid-gas (bubbly) slurry rheological behavior}

The sludge in Tank 241-SY-101 is believed to contain gas bubbles, which would affect the viscosity of the sludge. Under conditions where the sludge behaves as a fluid (that is, after it has yielded and begun to flow) the bubbles can be classified as spherical rather than dendritic, because the particles in the suspension would offer little resistance to bubble expansion.

\subsubsection{Single component}

For lack of other information, the solution, solids, and gas in the bubbly slurry are treated as single-component phases. Templates describing the dependence of rheological parameters on the void fraction are given in Equations (9.30) and (9.31).

\subsubsection{Templates for $\eta_{b}$, the bubbly slurry viscosity $(\mathrm{Pa}-s)$, and $\tau_{0 b}$, the bubbly slurry yield} stress $(\mathbf{P a})$

$$
\eta_{b}=\eta_{m}\left(1-\frac{\varepsilon_{d}}{\left(1-\varepsilon_{s}\right)}\right)^{-5 / 2}
$$

Range: all values of void fraction $\varepsilon_{\mathrm{d}}$ and solids fraction $\varepsilon_{\mathrm{s}}$

$$
\begin{aligned}
& \text { where } \eta_{m} \quad=\text { viscosity of the gas-free slurry (from Section 9.3) } \\
& \varepsilon_{\mathrm{d}} \quad=\text { volume fraction gas } \\
& \varepsilon_{\mathrm{s}} \quad=\text { volume fraction solids } \\
& \tau_{0 b}=\tau_{0}\left(1-\varepsilon_{d}\right)+\frac{\sigma}{D_{d}} f\left(\frac{\varepsilon_{d}}{\left(1-\varepsilon_{s}\right)}\right)
\end{aligned}
$$

(17) LaFemina, J.P., et al. 1995. Tank Waste Treatment Science: Report for the First Quarter FY 1995. TWRSPP-94-118. Letter report to Westinghouse Hanford Corporation. January 19, 1995. 
Range: all values of void fraction

$$
\begin{aligned}
& \text { where } \tau_{0}=\text { the dynamic yield stress of the gas-free slurry } \\
& \begin{aligned}
& \sigma=\text { surface tension at the gas-liquid interface } \\
& D_{d}=\text { bubble diameter } \\
& f() \quad= \text { a monotonically increasing function of } \varepsilon_{d} /\left(1-\varepsilon_{s}\right) \text {; experimentally } \\
&
\end{aligned}
\end{aligned}
$$

\subsubsection{Data}

An experimental study of the rheology of bubbly slurry is underway as part of the Flammable Gas Program (Gauglitz et al. 1994a). The data from this study are expected to clarify whether the presence of bubbles can best be modeled as increasing the viscosity in a rheological submodel that lacks yield stress, as shown in Equation (9.30), or as a change in the dynamic yield stress in a model that includes yield [Equation (9.31)], or both.

\subsubsection{Multi-component}

No data were found to predict the effect of multi-component chemical interactions on the rheological behavior of bubbly sludges and slurries.

\subsection{Summary of recent relevant experimental literature}

This section summarizes several papers or reports that studied concentrated suspensions. The object of these summaries is to show the rheological models and types of measurements that have been used in other work with suspension and sludge rheology.

(Cross 1965): His most applicable suspension was $40 \%$ limestone in water, with no data given on the size or shape of particles. The maximum packing fraction was not determined. Shear rates ranged from 10 to $15000 \mathrm{~s}^{-1}$. The viscometer was a cone and plate type. At low shear rates, Cross found an extremely high viscosity (or perhaps a yield stress), which made it impossible to use his model for correlation. At high shear rates the viscosity was $2 \mathrm{cP}$. 
(Faddick 1974): The suspensions were coal in water, with a solid specific gravity of 1.36 , concentrations from 12 to 46 vol\%, and three size ranges: 2300,100 , and $58 \mu \mathrm{m}$, with about $\pm 20 \%$ size variation. The maximum packing fraction was not determined. For the 58 and $100 \mu \mathrm{m}$ size slurries at $25^{\circ} \mathrm{C}$, the behavior was Newtonian below 15 vol\%; it then took on power-law characteristics. A yield stress developed at about 30 vol\%, and above 40 vol\%, a Bingham fluid was approximated. For all the concentrations tested, the viscosity of the suspension decreased with increasing temperature in the same way as for water, over a temperature range of 10 to $40^{\circ} \mathrm{C}$. Faddick used a power-law fit for the whole concentration range of $58-\mu \mathrm{m}$ data at $25^{\circ} \mathrm{C}$, even for those data where there appeared to be a yield stress. The structural index $\mathrm{n}$ decreased from 0.29 to 0.14 between 12 and 30 vol\%, then increased again to 0.7 at 43 vol\%. The consistency index $\mathrm{K}$ increased exponentially with volumetric concentration.

(Fow et al. 1986): The suspensions were simulated DST NCAW waste in supernatant. The concentrations were stated as $11-25$ vol\% centrifuged solids, and 0.6 to $6 \mathrm{wt} \%$ suspended solids. The particle size and shape were not stated and the maximum packing fraction was not determined. A rotary viscometer was used for shear rates between about 50 and $400 \mathrm{~s}^{-1}$. A Herschel-Bulkley model was fit to the data. The lower-concentration slurries showed no distinct yield stress and were powerlaw fluids at 25 and $100^{\circ} \mathrm{C}$, with the deviation from Newtonian behavior increasing with temperature. The most concentrated sludge showed yield stresses of 0.5 to $1 \mathrm{~Pa}$, with a higher yield stress at $100{ }^{\circ} \mathrm{C}$ than at $25^{\circ} \mathrm{C}$. At $100^{\circ} \mathrm{C}$, the concentrated sludge showed a higher yield stress for the increasingshear-rate than for the decreasing-shear-rate measurements. (The increasing-shear-rate measurements were made first.)

(Fam et al. 1989): The suspensions were phosphate mud in water, $0-11$ vol\%, with a solid specific gravity of 2.65 , mean diameter of $7-10 \mu \mathrm{m}$, maximum diameter of $50 \mu \mathrm{m}$, and irregular shapes. The maximum packing fraction was not determined. A rotary viscometer was used for shear rates between 50 and $2500 \mathrm{~s}^{-1}$. Data were fit to the Casson and Herschel-Bulkley (H-B) equations, with the Casson equation providing the better fit. The $\mathrm{H}-\mathrm{B}$ yield stress increased sharply at about 6 vol\%, from values of $0-2 \mathrm{~Pa}$ to values of 5 to $15 \mathrm{~Pa}$. The $\mathrm{K}$ and $\mathrm{n}$ of the power law part of the $\mathrm{H}-\mathrm{B}$ model also showed large changes at this point. The structural index $\mathrm{n}$ decreased monotonically over the concentration range; no minimum was observed. The index $n$ was near 1 (that is, a Bingham fluid) only for very low concentrations, less than 2 vol\%.

(Turian et al. 1992): The suspensions were bituminous coal in water, with concentrations from 24 to 50 vol\%, and four size ranges: $70-140 \mu \mathrm{m}, 50-100 \mu \mathrm{m}, 30-70 \mu \mathrm{m}$, and 1-30 $\mu \mathrm{m}$. The 
particles had sphericities of about 0.77 . Maximum packing fractions were found by centrifugation and were about $52 \mathrm{vol \%}$, except the finest particles (which had the broadest size range) had a maximum packing fraction of $63 \mathrm{vol} \%$. A capillary viscometer was used over a shear rate range of 1 to $4000 \mathrm{~s}^{-1}$. In the three large-particle samples, no yield stresses were measured at any of the concentrations, with either the capillary viscometer or a vane apparatus. These suspensions were Newtonian, with relative viscosities between 20 and 110 . The finest sample (1-30 $\mu \mathrm{m})$ was modeled using the Herschel-Bulkley, Bingham, and Casson models. Of these, the H-B model gave an unacceptably poor fit and the Casson model was best, giving $\eta_{0}$ values between 0.66 and $4.8 \mathrm{~Pa}$, and $\mathrm{K}$ values between 0.14 and $1.0 \mathrm{P}$, depending on concentration.

(Husband et al. 1993): The suspensions were $\mathrm{CaCO}_{3}$ in polyisobutylene, with a concentration range from 0 to $57 \mathrm{vol} \%$, and $98 \%$ of the particles between 10 and $40 \mu \mathrm{m}$. The limiting pure-fluid viscosity was $65-160 \mathrm{kPa}-\mathrm{s}$ at low shear rate. The maximum packing fraction was not determined. For shear rates between $10^{-6}$ and $8 \times 10^{-3} \mathrm{~s}^{-1}$, creep tests were used to measure elongational viscosity. Shear viscosity was calculated from the elongational viscosity data. A cone and plate viscometer was used between $2 \times 10^{-3}$ and $1 \mathrm{~s}^{-1}$. The elongational low-shear viscosities were $0.2-6 \mathrm{MPa}$-s at 38 vol\% solids or less, and were $900-6000 \mathrm{MPa}-\mathrm{s}$ above $50 \mathrm{vol} \%$. Static yield stresses of 0.5 to $26 \mathrm{kPa}$ appeared at 50 vol\% and above. No evidence of a dynamic yield stress was found in any of the suspensions.

(Kalyon et al.1993): The suspension contained 76.5 vol\% bimodal aluminum and ammonium sulfate in hydroxyl-terminated polybutadiene (HTPB). Of the aluminum particles, $90 \%$ were less than $29 \mu \mathrm{m}$ in size; $25 \%$ of the ammonium sulfate was $20 \mu \mathrm{m}$, and $75 \%$ was $200 \mu \mathrm{m}$ with oblong and spherical shapes. The HTPB viscosity was $1.3 \mathrm{~Pa}$-s. The maximum packing fraction was not determined. A parallel disk viscometer was used for shear rates from 0.005 to $0.1 \mathrm{~s}^{-1}$, and a capillary viscometer was used from 0.1 to $1000 \mathrm{~s}^{-1}$, with flow visualization observations to determine wall slip. Wall slip correlated very strongly with shear stress. The combined parallel-disk and capillary viscometer data exhibited shear thinning at rates between 30 and $3000 \mathrm{~s}^{-1}$, and two different viscosities in the 0.001-0.05 s-1 and 30-3000 s-1 ranges. A Herschel-Bulkley model fit gave $\mathrm{n}=0.37$, $\mathrm{K}=10,300 \mathrm{~Pa}-\mathrm{s}^{0.37}$, yield stress not stated, and quality of fit not stated. 


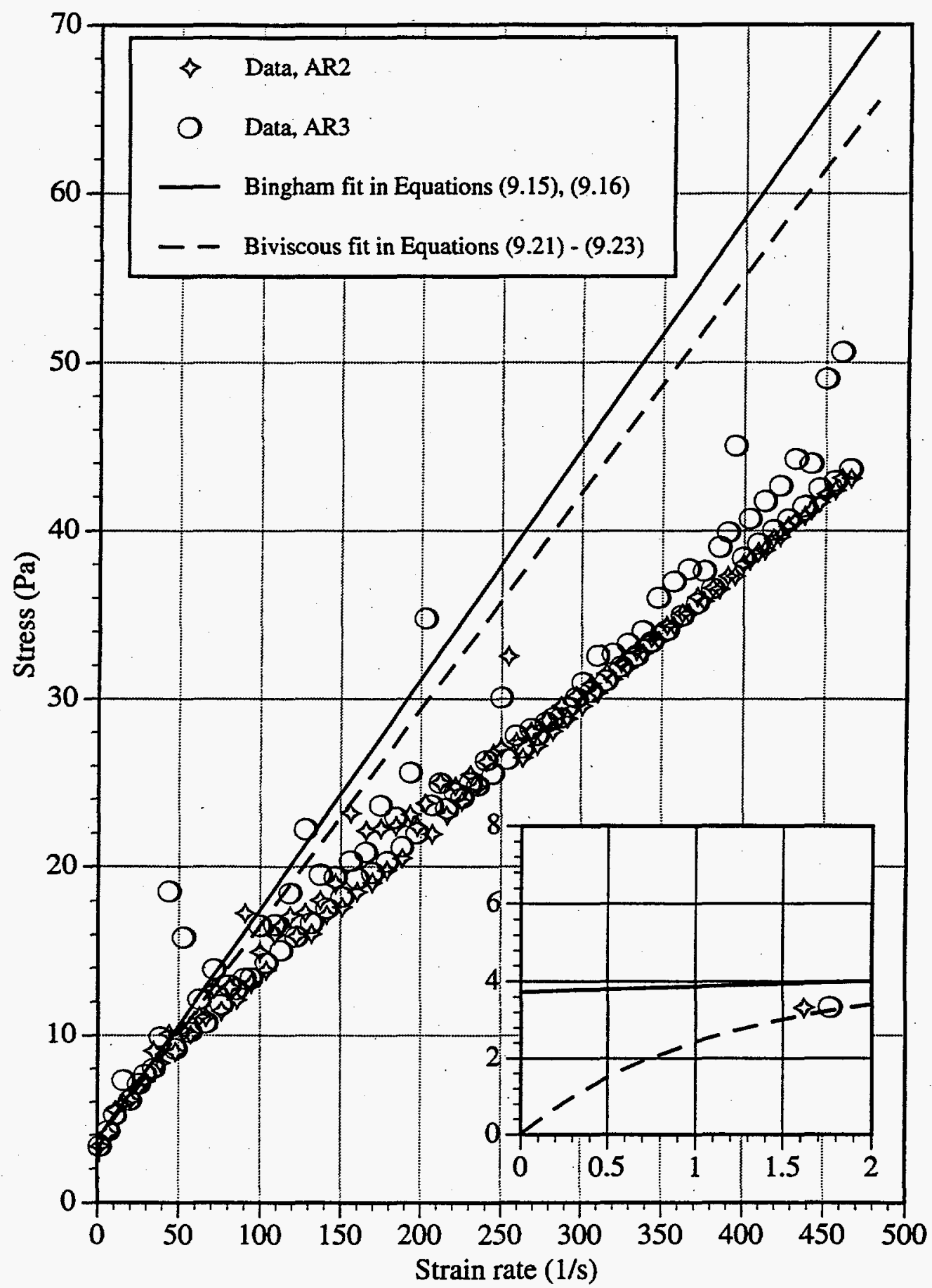

Figure 9:1. Rheological data and model fits (in the 0 to $50 / \mathrm{s}$ range) for SY-101 sludge at $0 \%$ dilution and $50^{\circ} \mathrm{C}$. 


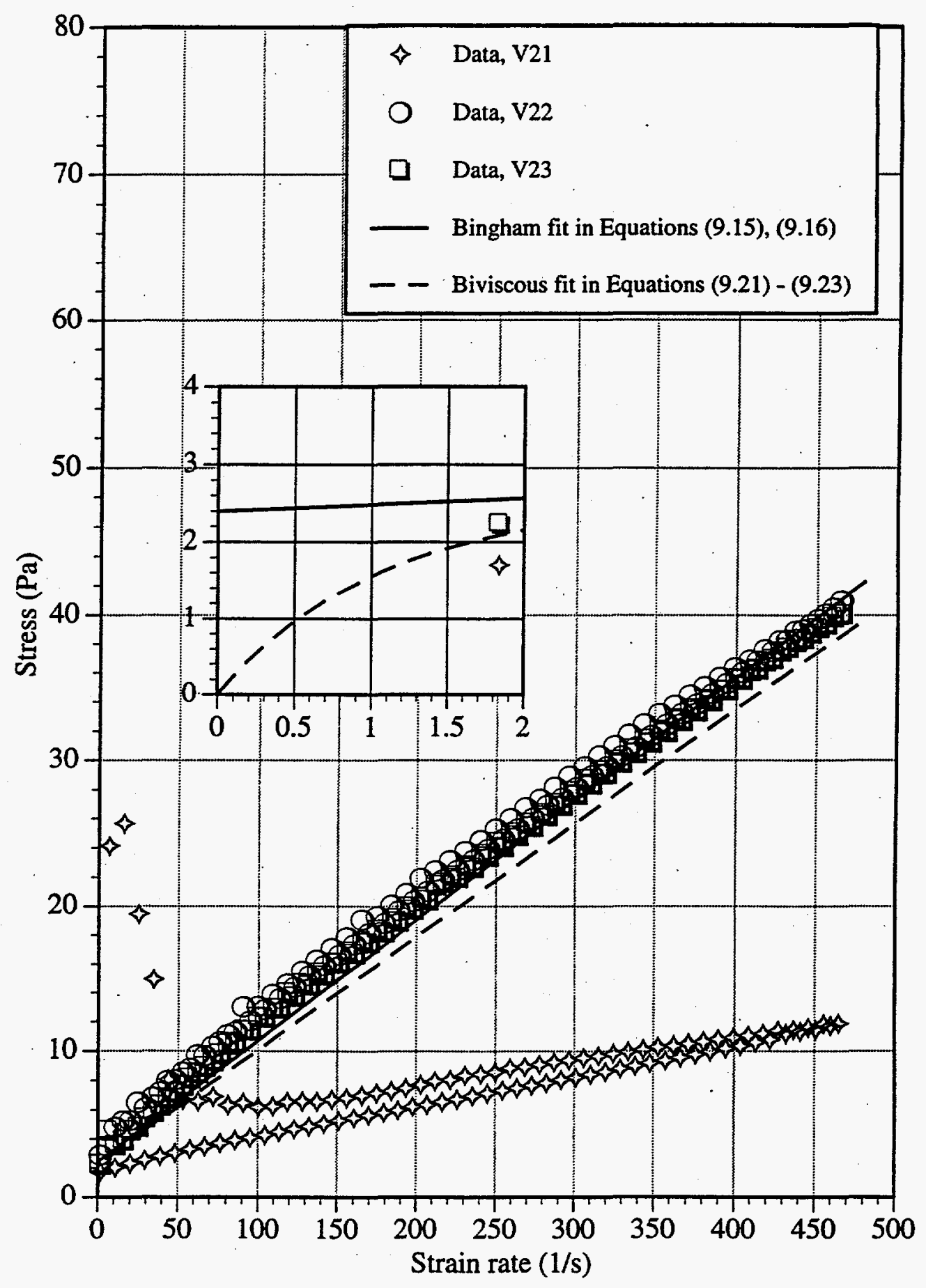

Figure 9.2. Rheological data and model fits (in the 0 to $50 / \mathrm{s}$ range) for SY-101 sludge at $10 \%$ dilution and $50^{\circ} \mathrm{C}$. 


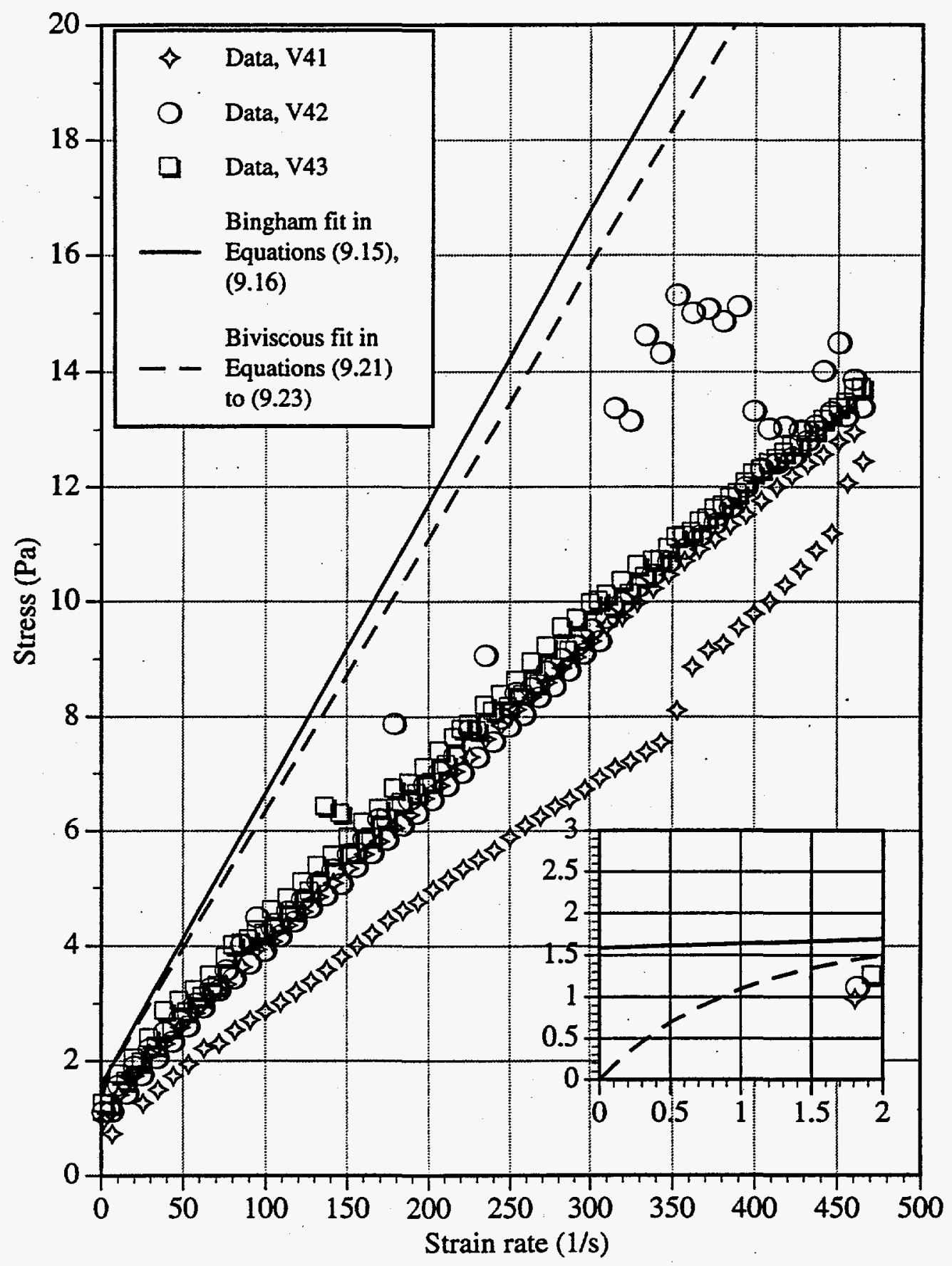

Figure 9.3. Rheological data and model fits (in the 0 to $50 / \mathrm{s}$ range) for SY-101 sludge at $20 \%$ dilution and $50^{\circ} \mathrm{C}$. 


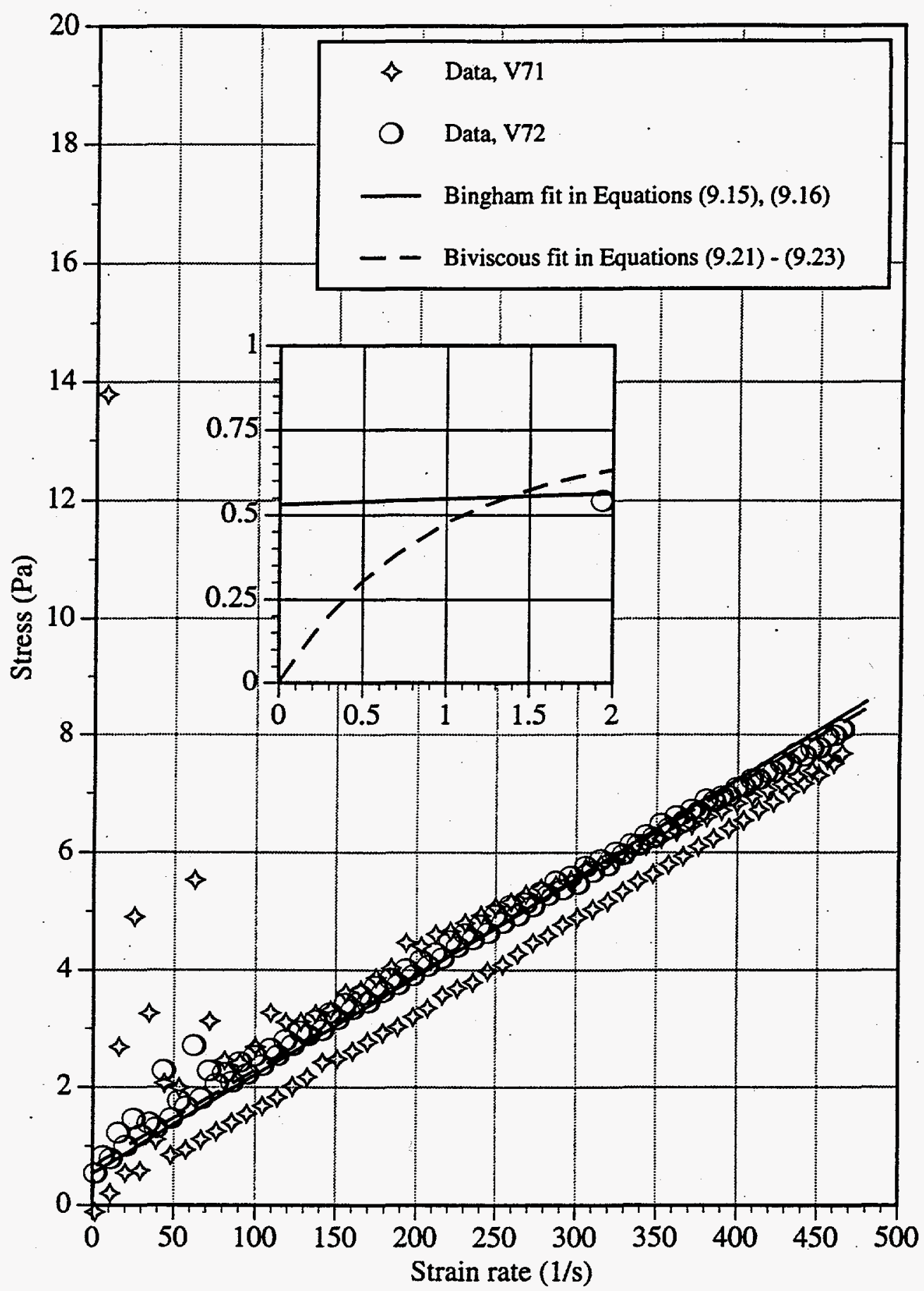

Figure 9.4. Rheological data and model fits (in the 0 to $50 / \mathrm{s}$ range) for SY-101 sludge at $35 \%$ dilution and $50^{\circ} \mathrm{C}$. 


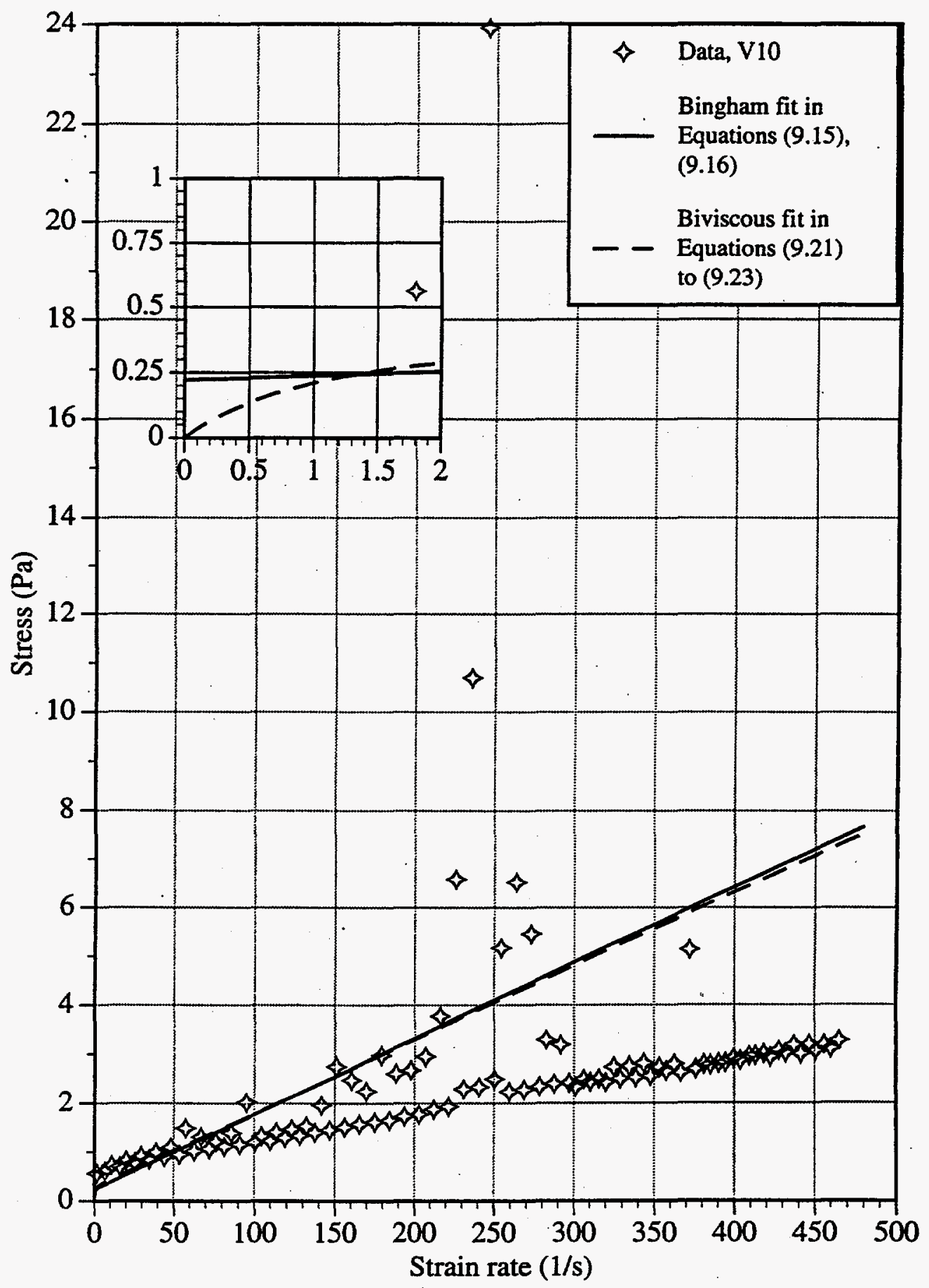

Figure 9.5. Rheological data and model fits (in the 0 to $50 / \mathrm{s}$ range) for SY-101 sludge at $50 \%$ dilution and $50^{\circ} \mathrm{C}$. 


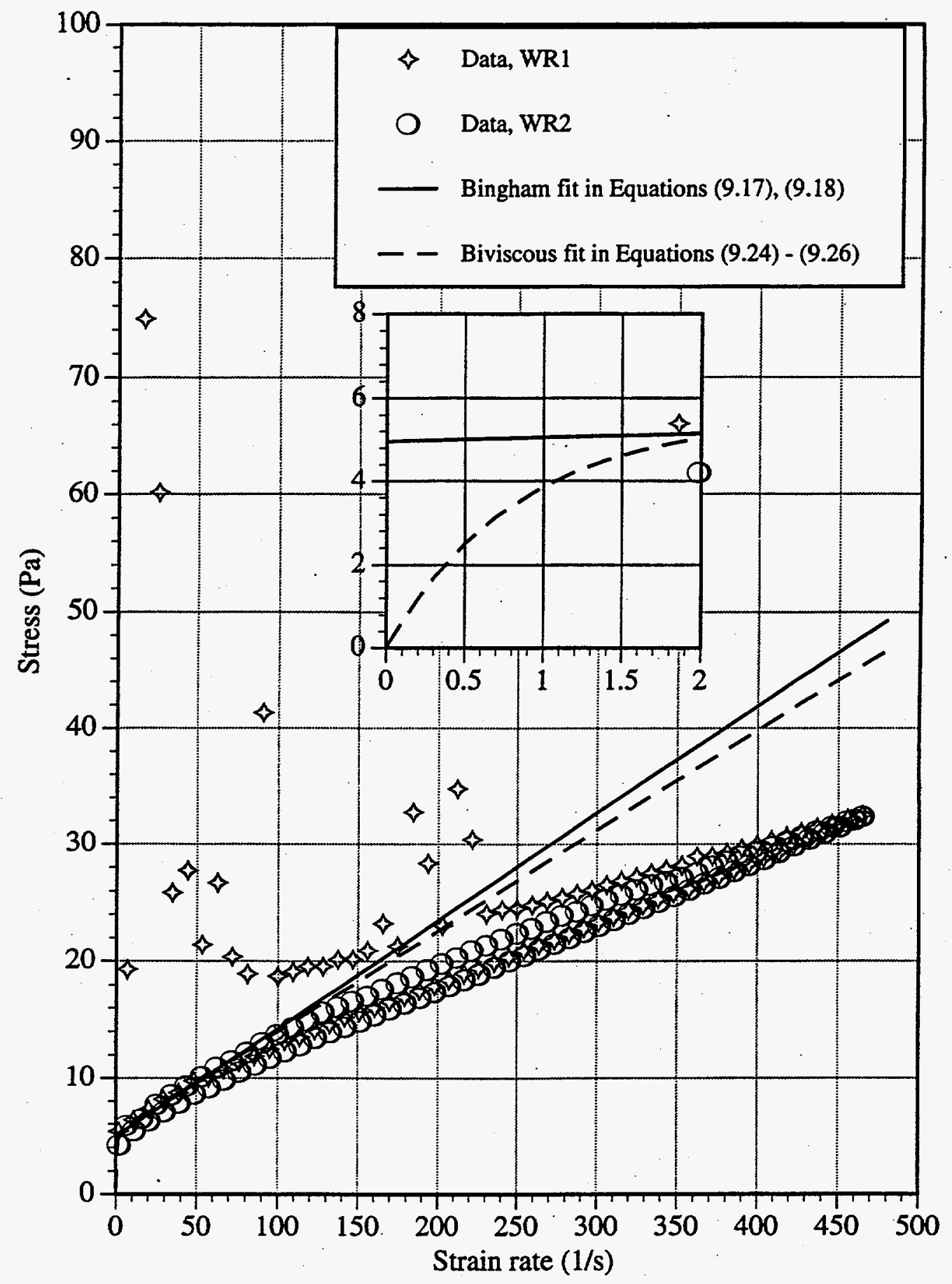

Figure 9.6. Rheological data and model fits (in the 0 to $50 / \mathrm{s}$ range) for SY-101 sludge at $0 \%$ dilution and $70^{\circ} \mathrm{C}$. 


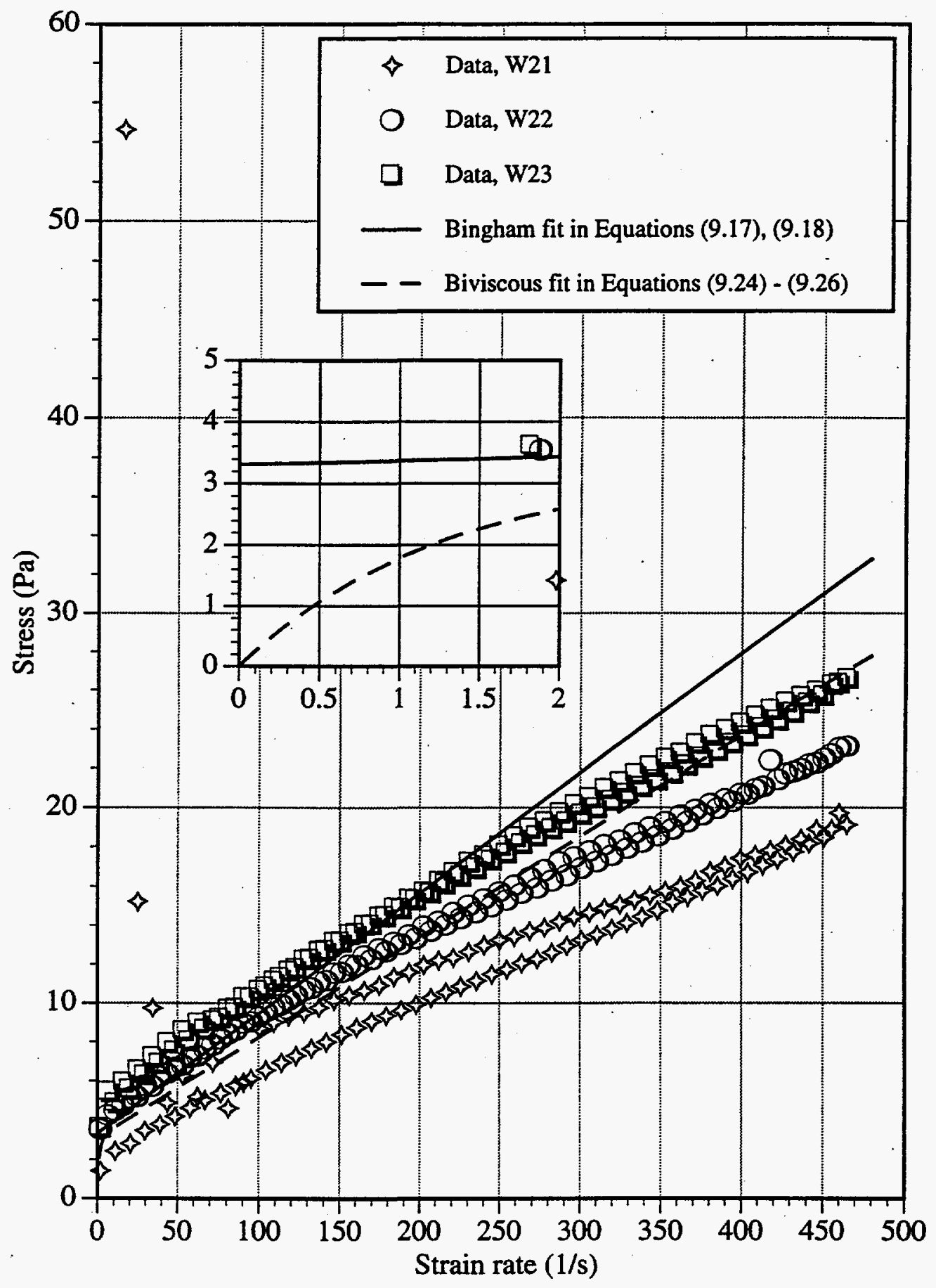

Figure 9.7. Rheological data and model fits (in the 0 to $50 / \mathrm{s}$ range) for SY-101 sludge at $10 \%$ dilution and $70^{\circ} \mathrm{C}$. 


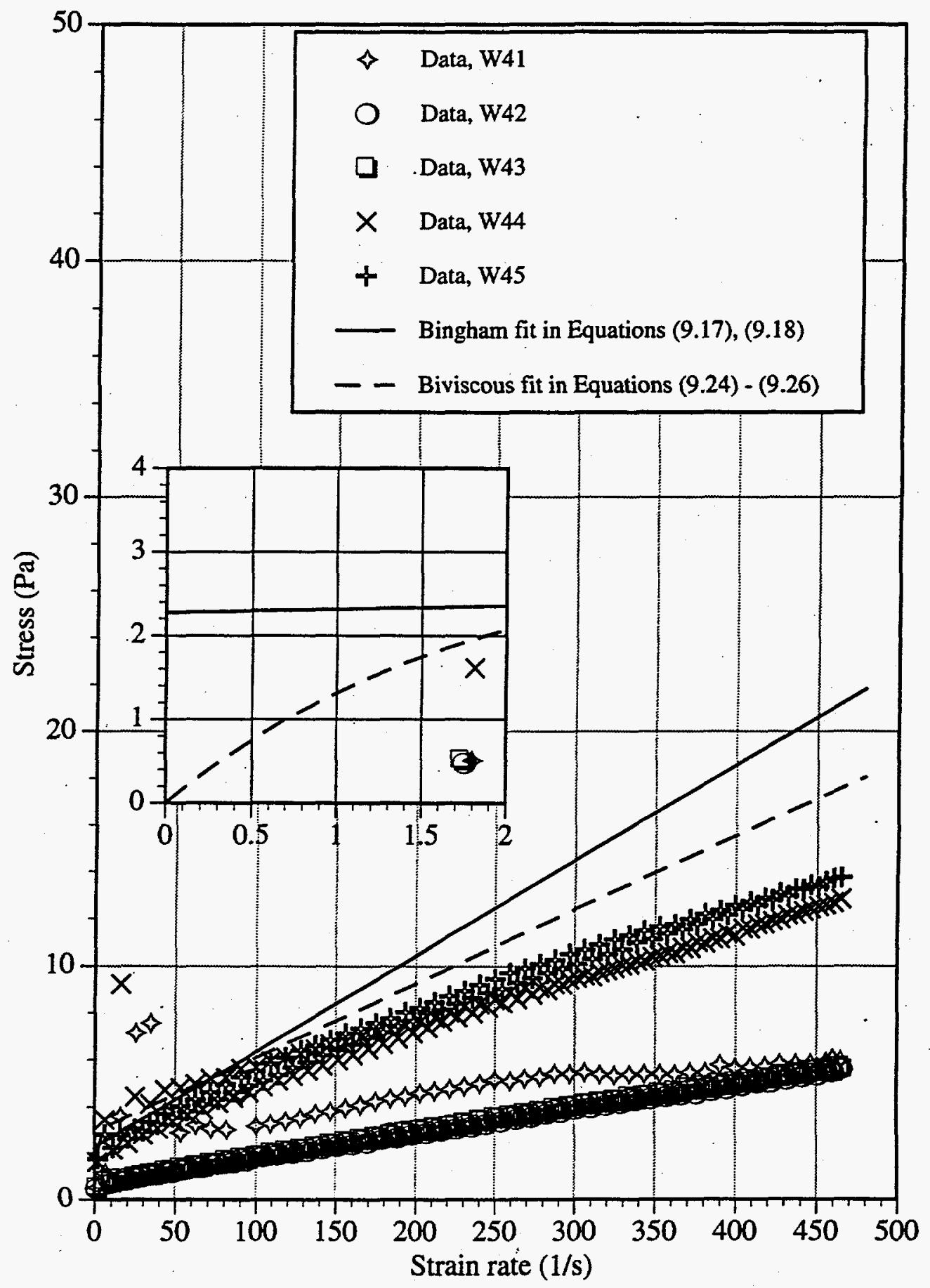

Figure 9.8. Rheological data and model fits (in the 0 to $50 / \mathrm{s}$ range) for SY-101 sludge at $20 \%$ dilution and $70^{\circ} \mathrm{C}$. 


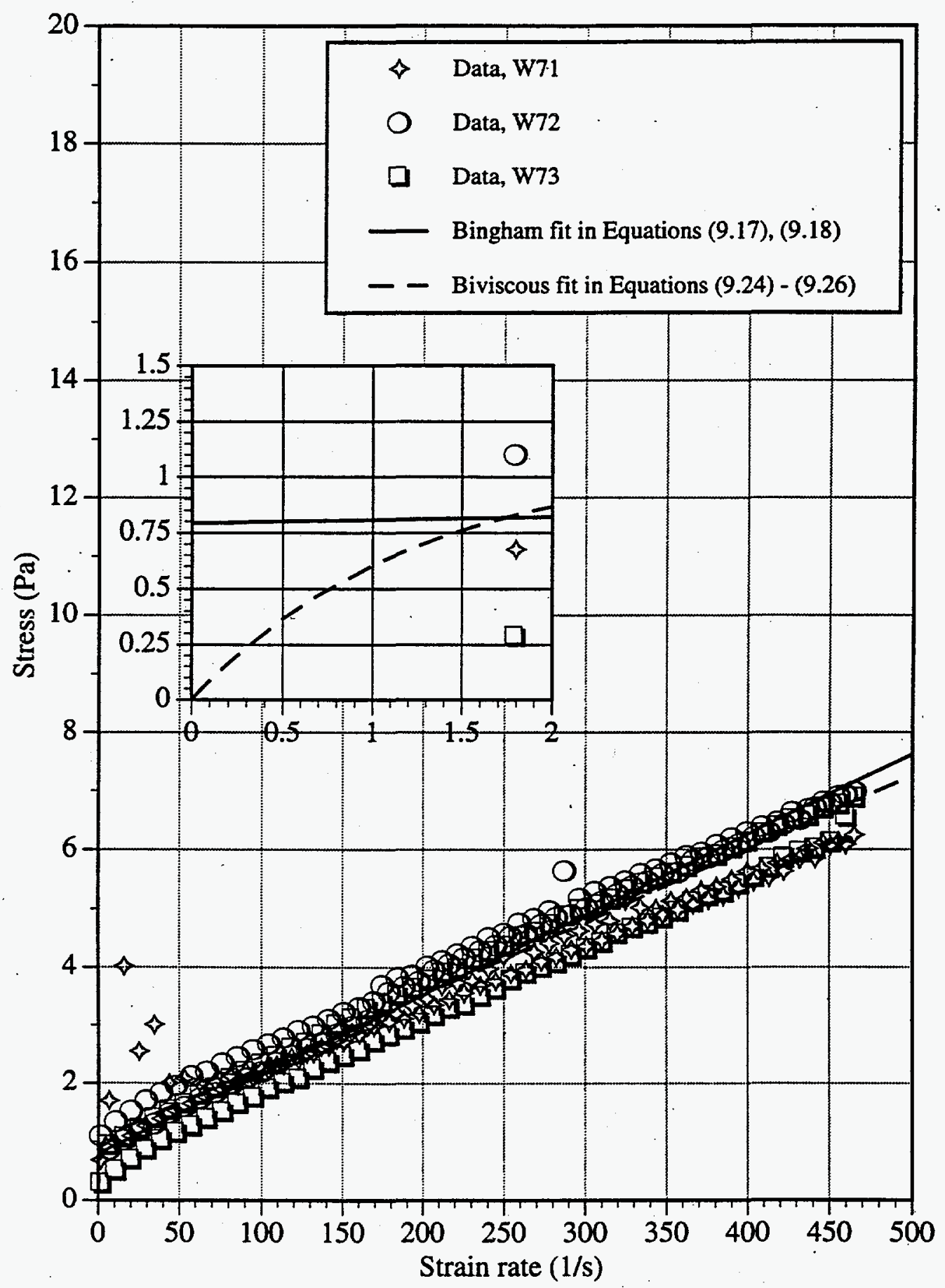

Figure 9.9. Rheological data and model fits (in the 0 to $50 / \mathrm{s}$ range) for SY-101 sludge at $35 \%$ dilution and $70^{\circ} \mathrm{C}$. 


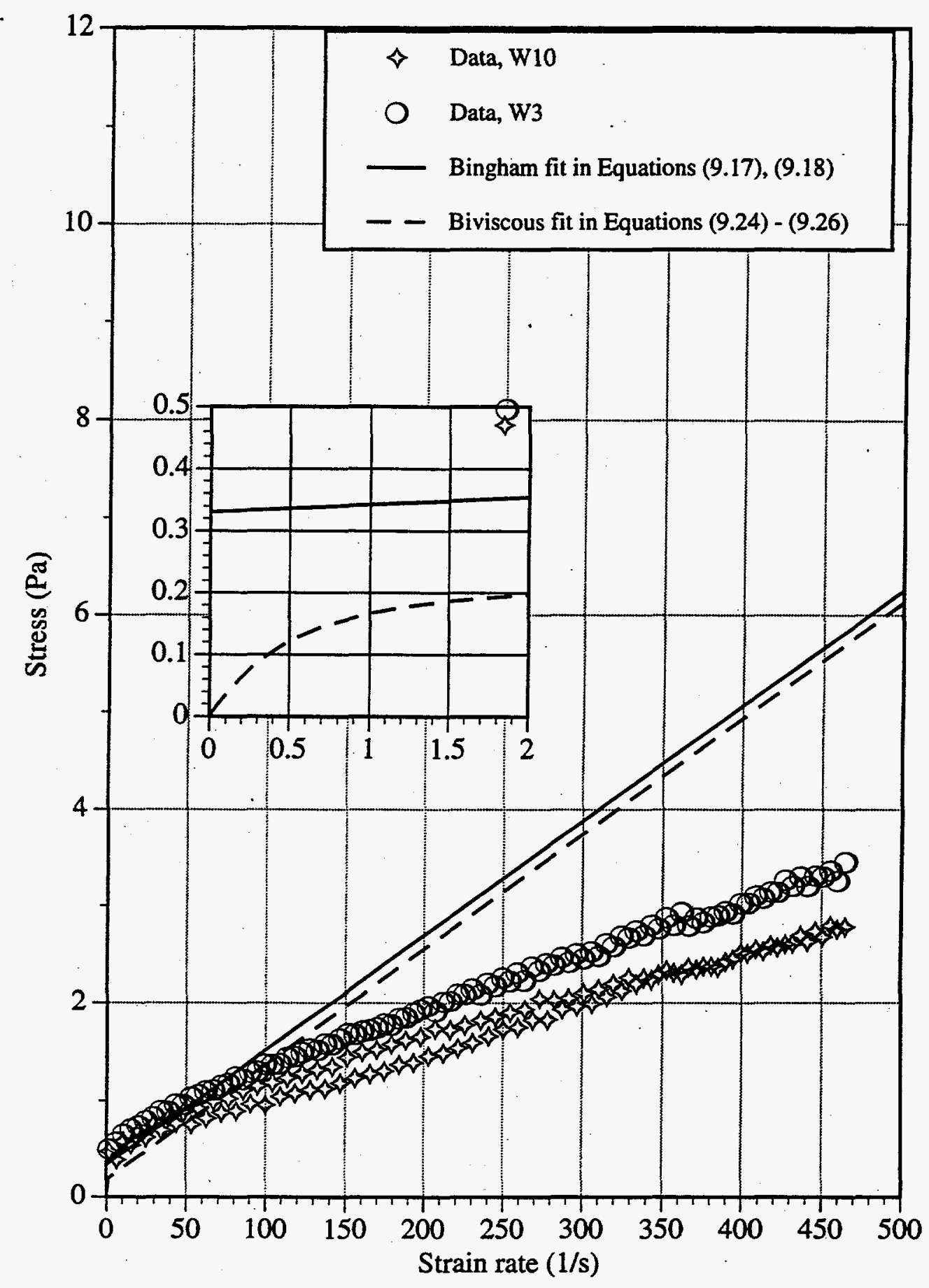

Figure 9.10. Rheological data and model fits (in the 0 to $50 / \mathrm{s}$ range) for SY-101 sludge at $50 \%$ dilution and $70^{\circ} \mathrm{C}$. 




Figure 9:11. Rheological data and model fits in the 0 to $50 / \mathrm{s}$ range) for SY-101 sludge at $0 \%$ dilution and $90^{\circ} \mathrm{C}$. 




Figure 9.12. Rheological data and model fits (in the 0 to $50 / \mathrm{s}$ range) for SY-101 sludge at $10 \%$ dilution and $90^{\circ} \mathrm{C}$. 


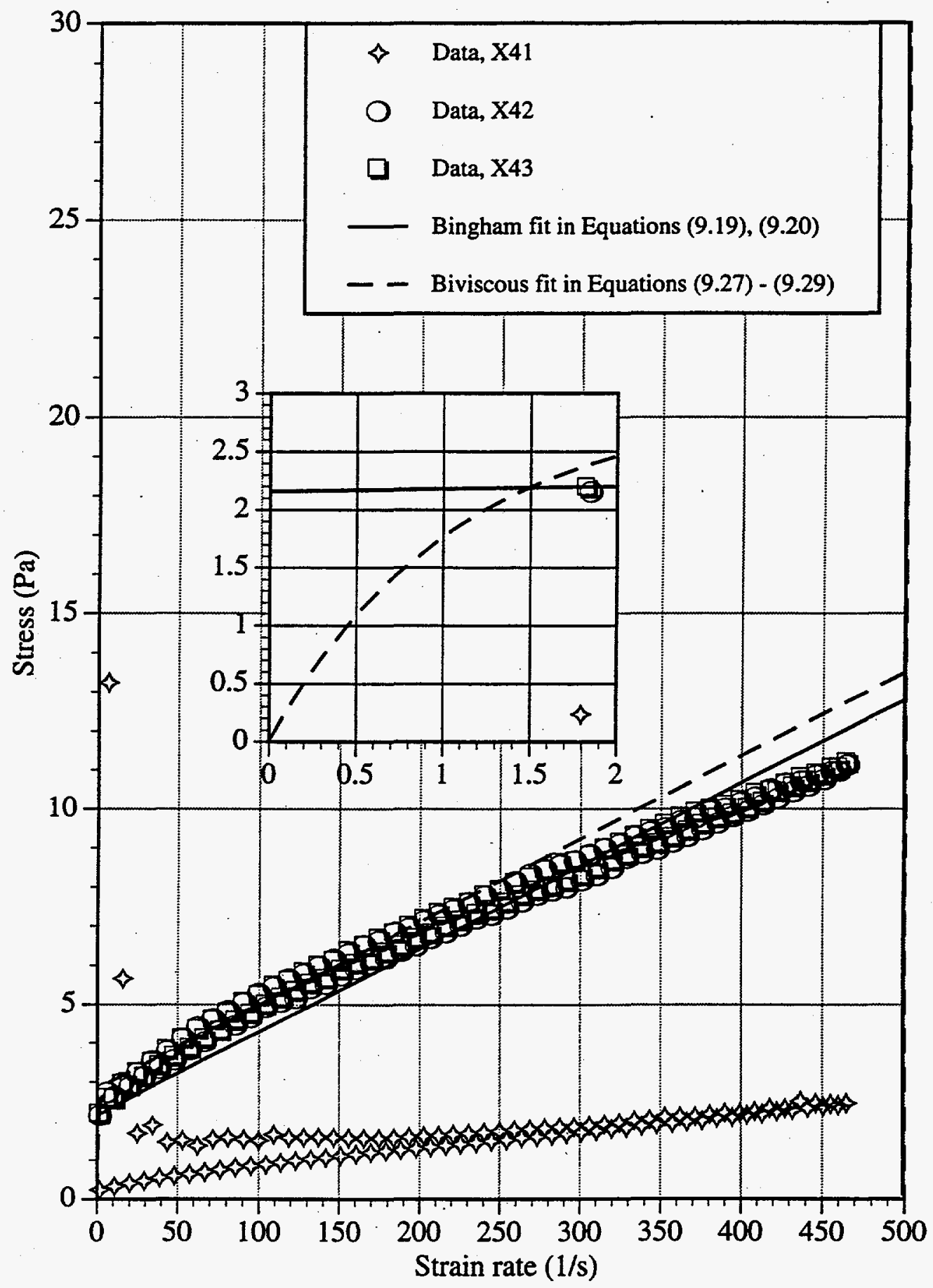

Figure 9.13. Rheological data and model fits (in the 0 to $50 / \mathrm{s}$ range) for SY-101 sludge at $20 \%$ dilution and $90^{\circ} \mathrm{C}$. 


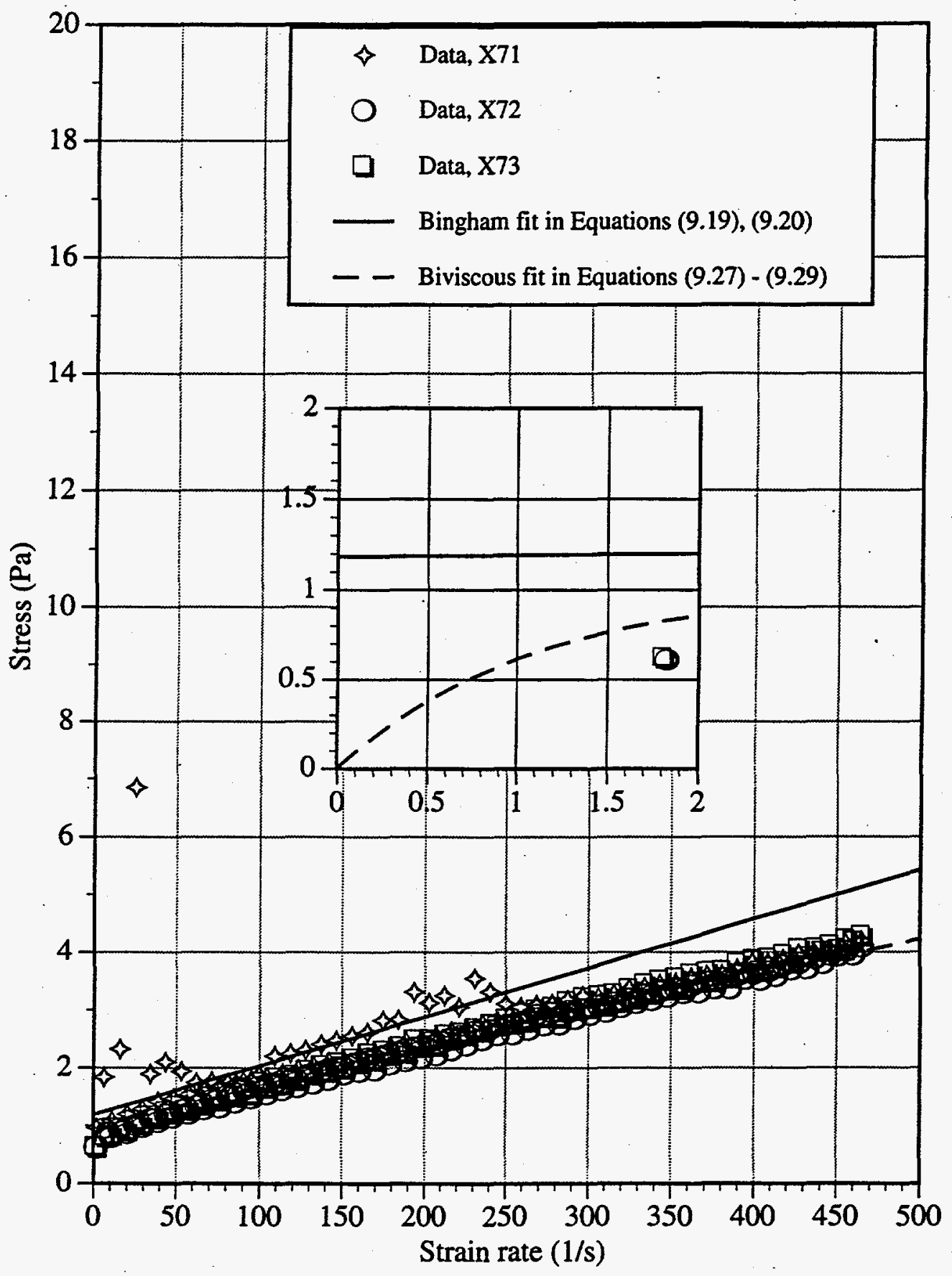

Figure 9:14. Rheological data and model fits (in the 0 to $50 / \mathrm{s}$ range) for SY-101 sludge at $35 \%$ dilution and $90^{\circ} \mathrm{C}$. 


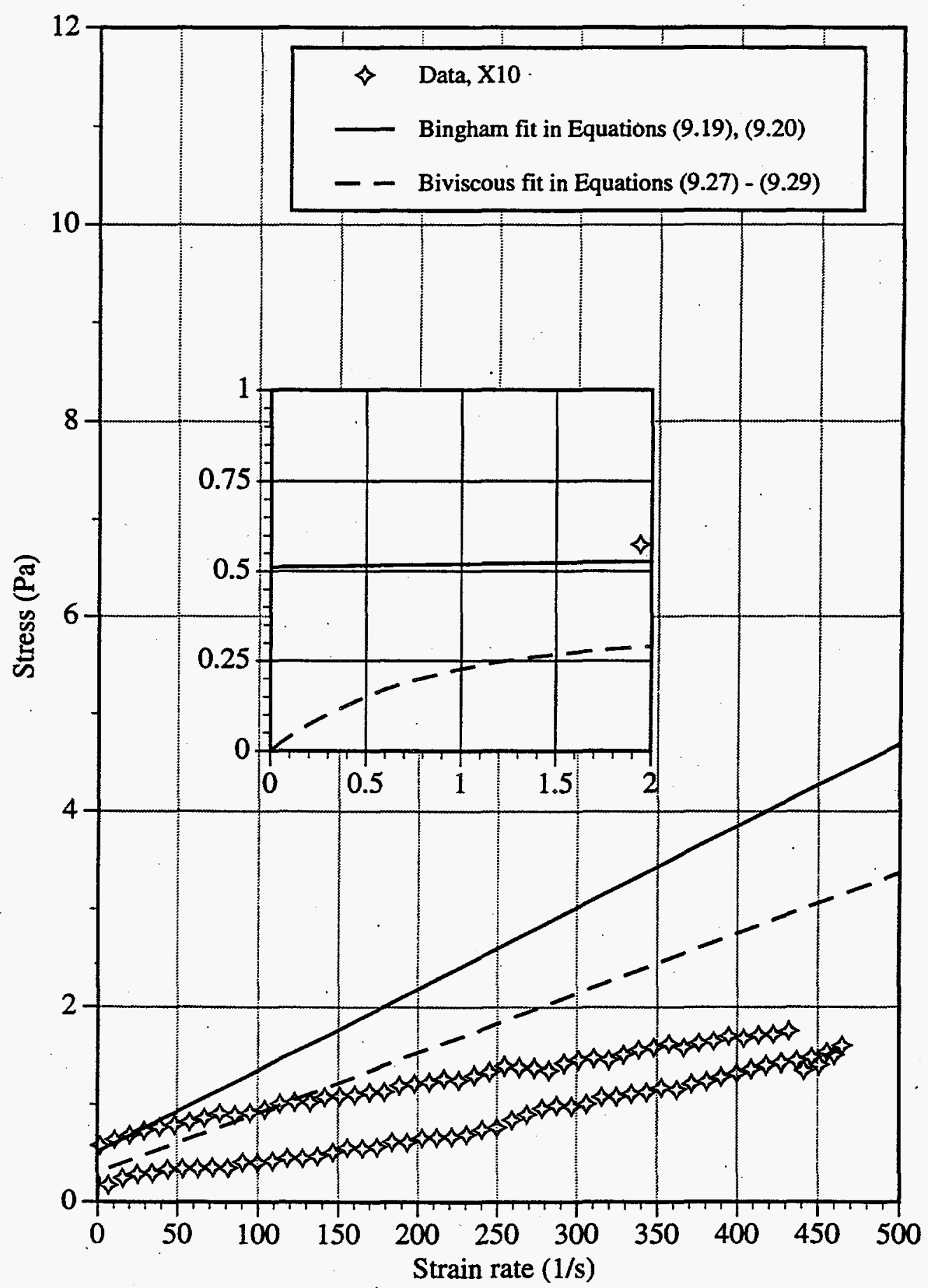

Figure 9.15. Rheological data and model fits (in the 0 to $50 / \mathrm{s}$ range) for SY-101 sludge at $50 \%$ dilution and $90^{\circ} \mathrm{C}$. 


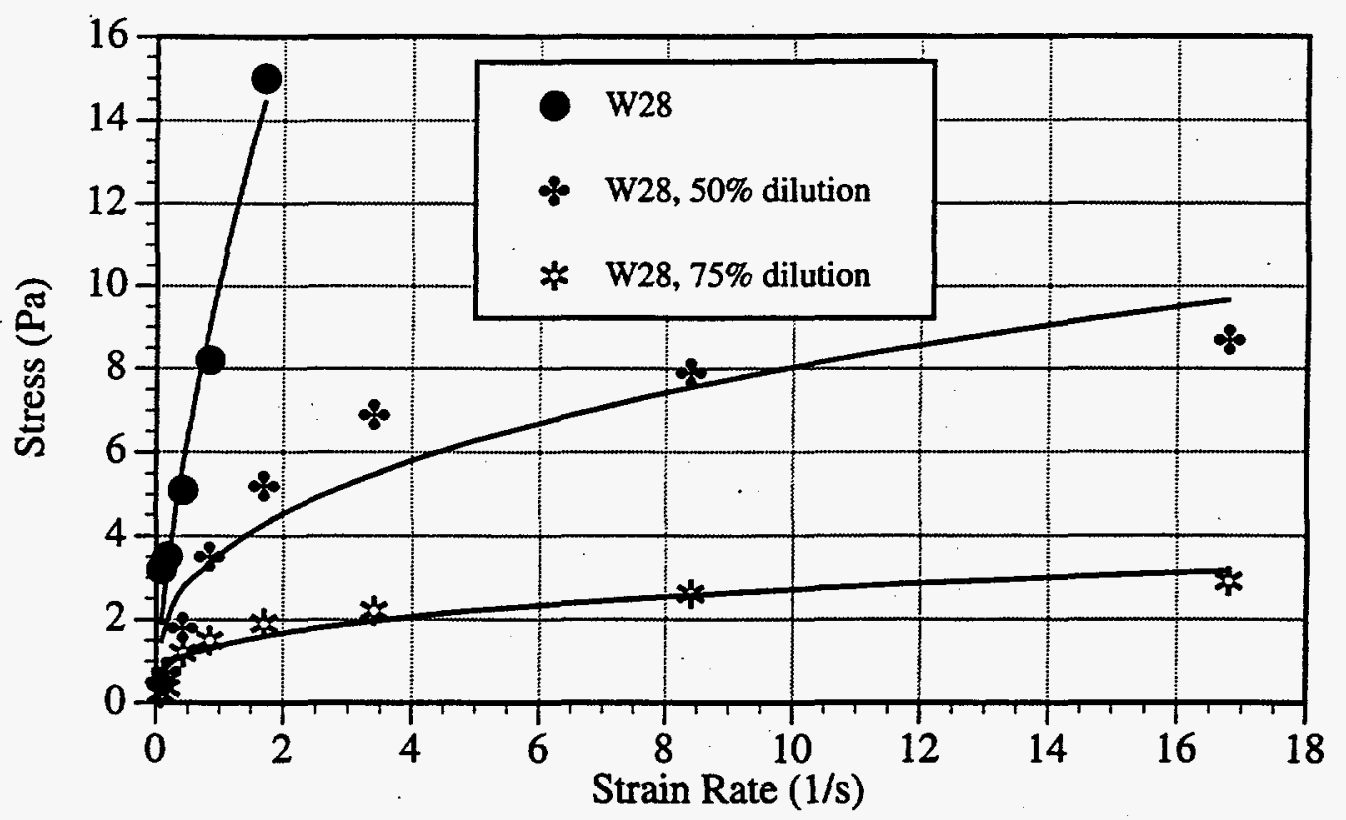

Figure 9.16. Tank W-28 data as fitted by a power-law model.

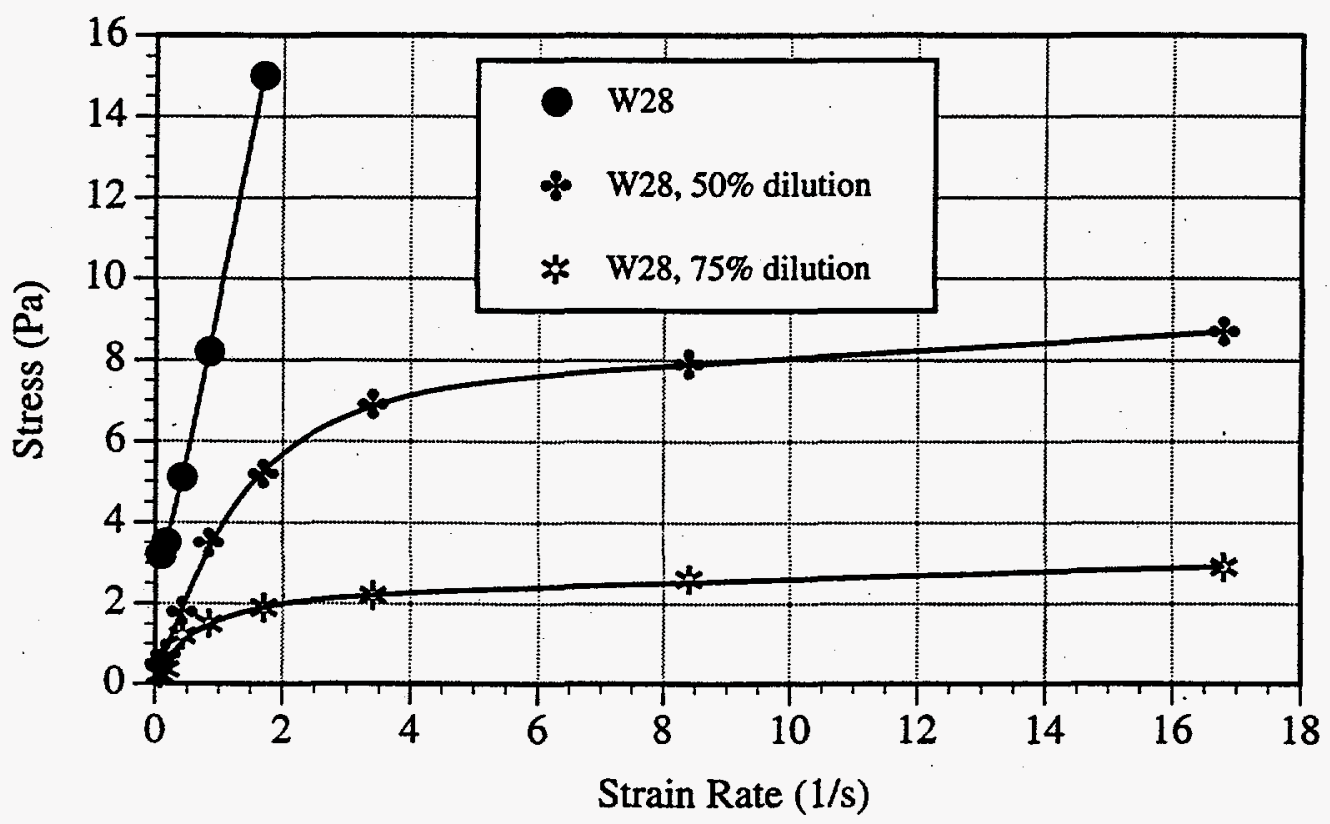

Figure 9.17. Tank W-28 data as fitted by the Cross model. 


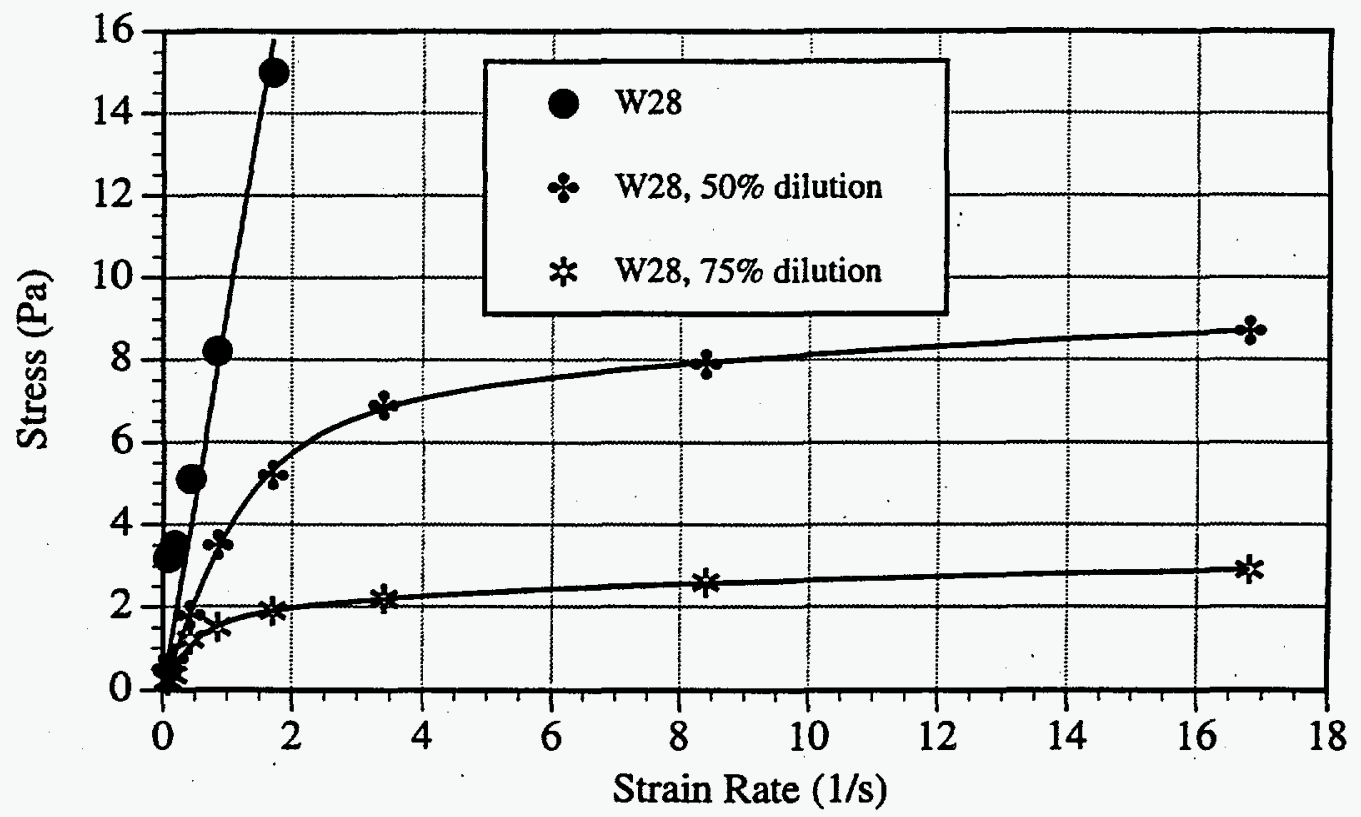

Figure 9.18. Tank W-28 data as fitted by the Carreau model.

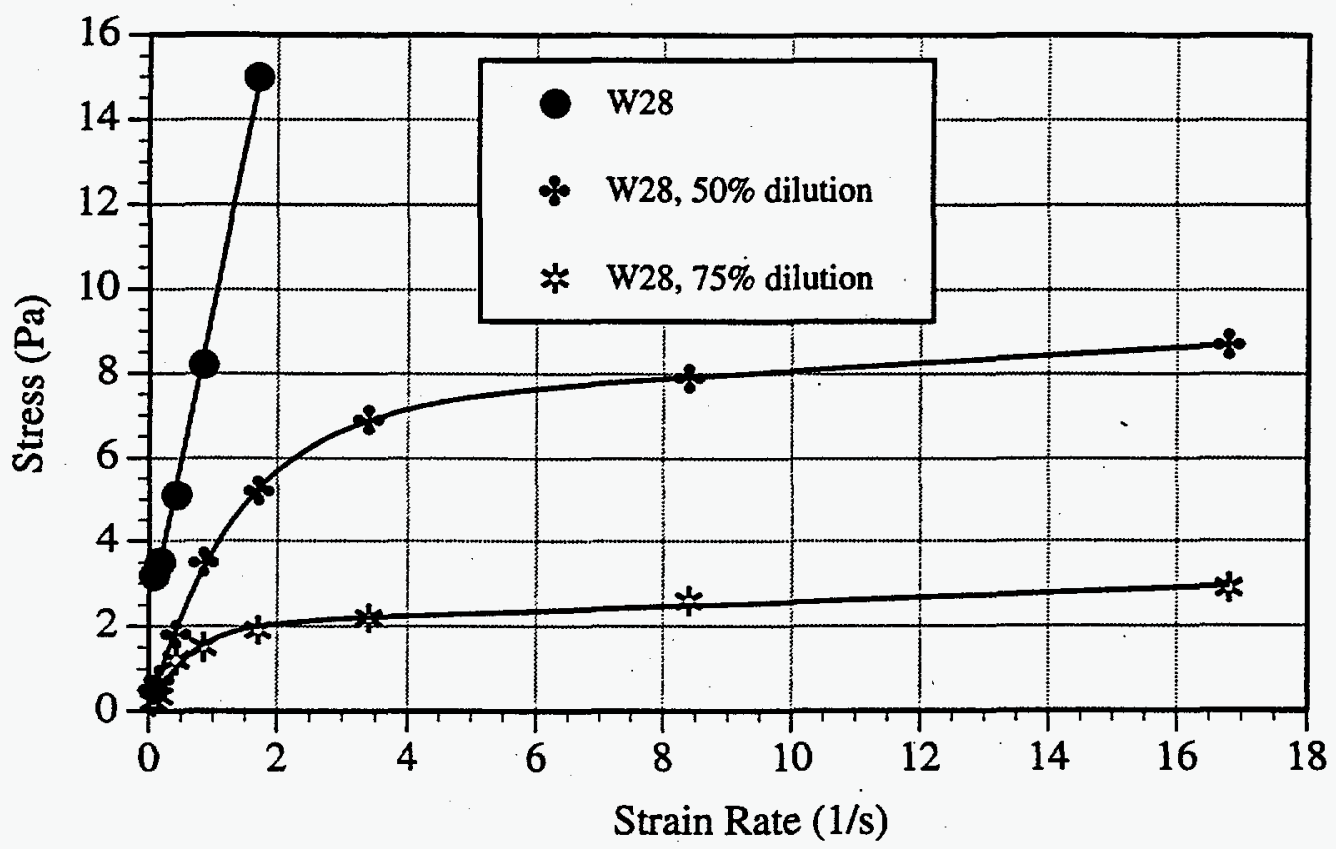

Figure 9.19. Tank W-28 data as fitted by a biviscous model. 


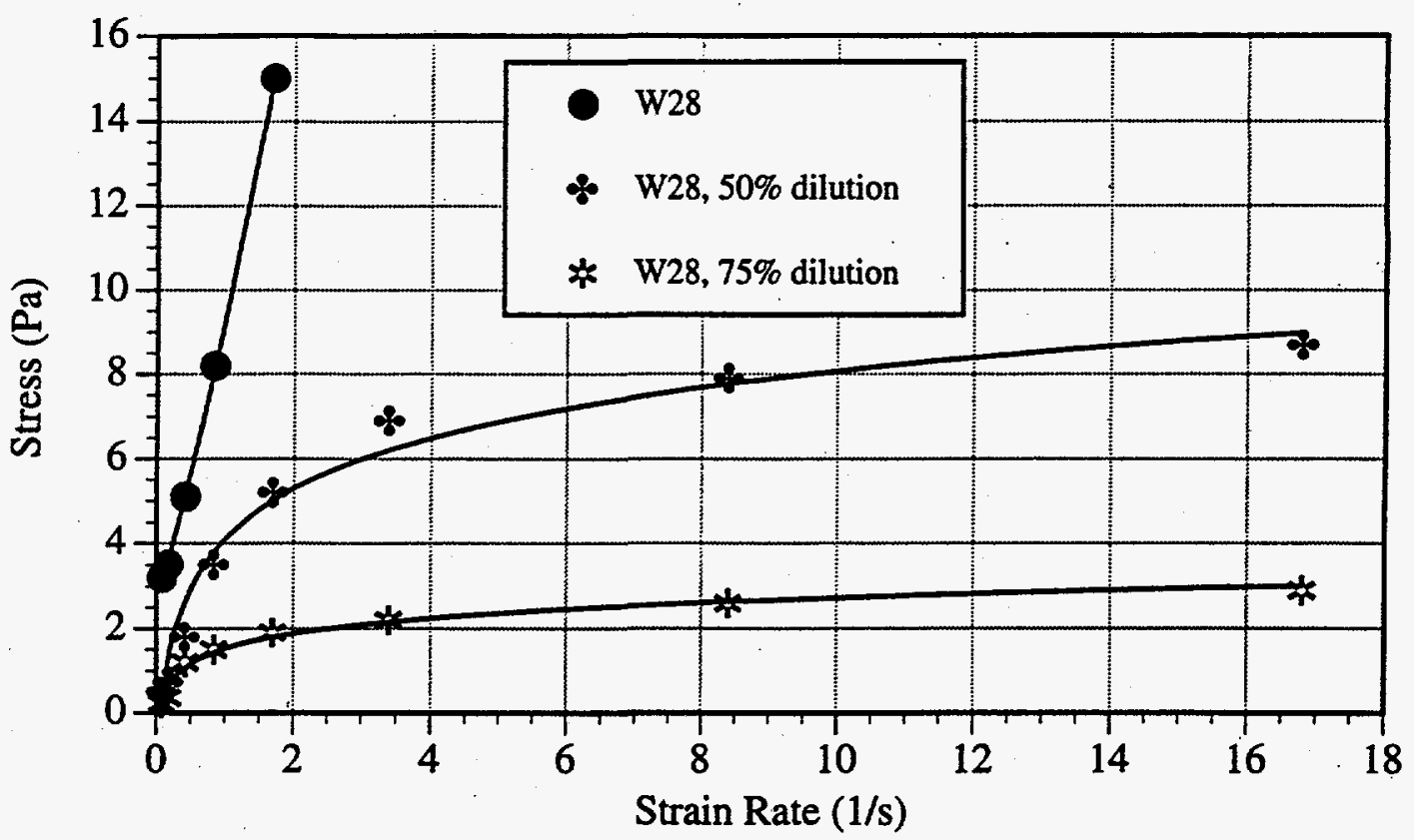

Figure 9.20. Tank W-28 data as fitted by the Herschel-Bulkley model.

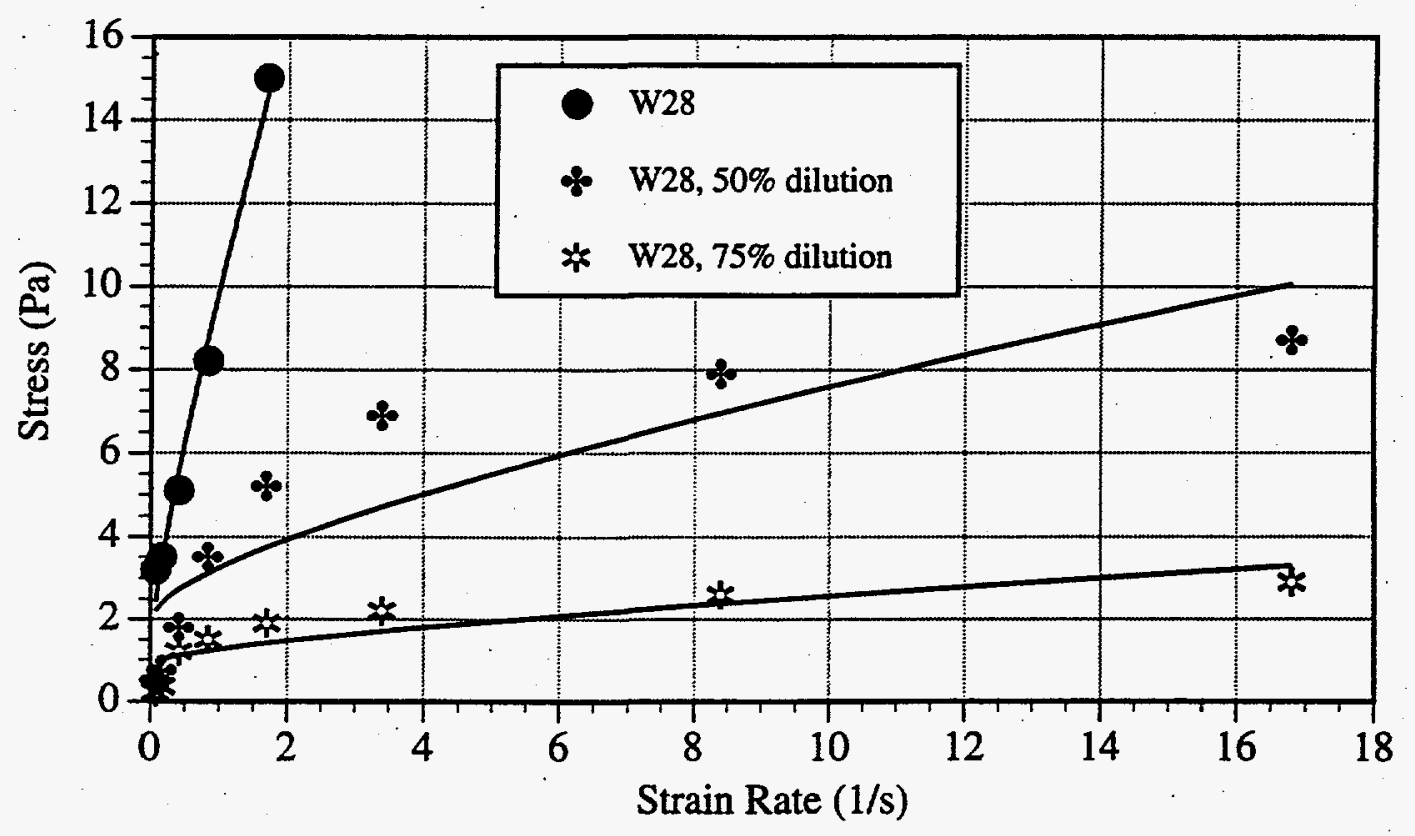

Figure 9.21. Tank W-28 data as fitted by the Casson model. 




Figure 9.22. Tank W-28 data as fitted by the Gay model. 


\subsection{Heat Capacity}

The heat capacity of the sludge in the waste tanks determines the time scale over which temperature variations occur as a result of heat generation and heat transfer. This chapter begins by providing correlations and other submodels for the heat capacity of single phases, the gas in the bubbles (Section 10.1), the solution liquid (Section 10.2), and the solids (Section 10.3). Section 10.4 specifies a submodel for the gasless slurry heat capacity, and Section 10.5 discusses the bubbly slurry heat capacity.

\subsection{Single-phase gas heat capacity}

Gas is believed to be present in the sludge primarily in the form of small dendritic or spherical bubbles.

\subsubsection{Single component}

The gas in the tanks is at near-ambient temperatures and pressures, and its heat capacity can safely be treated as dependent on temperature but not on pressure. The multi-component waste gas can be represented by one of its components, as in Equation (10.1), if that species is sufficiently predominant.

\subsubsection{Template for $\mathrm{c}_{\mathrm{pi}}$, the heat capacity of a gaseous species $(\mathrm{J} / \mathrm{kg} \mathrm{K})$}

$$
c_{p i}=a_{0}+a_{3} T+a_{6} T^{2}+a_{7} T^{3}
$$

Range: temperature $273-373 \mathrm{~K}$; pressure less than 10 atm

$$
\text { where } \begin{aligned}
a_{k} & =\text { coefficients found by correlation } \\
T & =\text { temperature }(K)
\end{aligned}
$$

\subsubsection{Data}

Table 10.1 contains the heat capacity correlation constants for the predominant gases in the waste gas: hydrogen, nitrogen, nitrous oxide, and ammonia. (The gas composition is described in Section 4.1.1.2.) The heat capacity correlations are taken from Appendix E of Himmelblau (1974). No correlation accuracies are given in the reference. 
Table 10.1. Heat capacity correlations $(\mathrm{J} / \mathrm{kg} \mathrm{K}$ ) for gases present in the waste gas. The correlations all use the template in Equation (10.1).

\begin{tabular}{llllll} 
Gas & & $a_{0}$ & $a_{3}$ & $a_{6} * 106$ & $a_{7}^{*} 10^{6}$ \\
\hline $\mathrm{H}_{2}$ & & 13330 & 2.156 & -160 & \\
$\mathrm{~N}_{2}$ & & 974.9 & 0.2222 & -33.91 & \\
$\mathrm{~N}_{2} \mathrm{O}$ & & 547.2 & 1.331 & -808.6 & 0.240 \\
$\mathrm{NH}_{3}$ & 1618 & 1.504 & 581.7 & -0.393
\end{tabular}

\subsubsection{Multi-component}

Alternately, the gas can be represented as a multi-component mixture with a mass-weighted average heat capacity, as in Equation (10.2).

\subsubsection{Template for $c_{p d}$, the gas heat capacity $(\mathrm{J} / \mathrm{kg} \mathrm{K})$}

$$
c_{p d}=\frac{\sum_{i=1}^{N} x_{i} W_{i} c_{p i}}{\sum_{i=1}^{N} x_{i} W_{i}}
$$

Range: temperature greater than $250 \mathrm{~K}$; pressure less than 10 atm (Perry and Chilton, 1973)

$$
\text { where } \begin{array}{ll}
c_{\mathrm{pi}} & =\text { heat capacity of gaseous species } \mathrm{i}(\mathrm{J} / \mathrm{kg} \mathrm{K}) \\
\mathrm{x}_{\mathrm{i}} & =\text { mole or volume fraction of the } \mathrm{i}-\mathrm{th} \text { species of } \mathrm{N} \text { gaseous species } \\
\mathrm{W}_{\mathrm{i}} & =\text { molecular weight of the } \mathrm{i} \text {-th gaseous species }(\mathrm{kg} / \mathrm{gmol})
\end{array}
$$

\subsubsection{Data}

The molecular weights and composition data for the gases in Tank 241-SY-101 wastes are the same as those given in Section 4.1.1.2. The minor components of the gas can be considered to have little effect, because the major components are the gases listed in Table 10.1. 


\subsection{Single-phase liquid heat capacity}

The waste tank supernatant solution is a combination of $\mathrm{NaNO}_{3}, \mathrm{NaNO}_{2}$, and other nitrate and sodium salts. The nitrate and nitrite salts probably account for most of the heat capacity characteristics of the supernatant.

\subsubsection{Single component}

As an approximation, the supernatant liquid in Tank 241-SY-101 and other Hanford waste tanks can be treated as a single-component solution, as in Equation (10.3), using one of the major aqueous species as a surrogate for the other components.

\subsubsection{Template for $\mathrm{c}_{\mathrm{pc}}$, the liquid heat capacity $(\mathrm{J} / \mathrm{kg} \mathrm{K})$}

$$
c_{p c}=a_{0}+a_{1} C+a_{3} T+a_{8} C^{2} T
$$

Range: $293-373 \mathrm{~K} ; 0$ to 100 wt\% solute concentration

$$
\text { where } \begin{array}{ll}
a_{k} & =\text { coefficients obtained by data correlation } \\
\mathrm{C} & =\text { concentration of solute in weight percent of the total solution } \\
\mathrm{T} & =\text { temperature }(\mathrm{K})
\end{array}
$$

\subsubsection{Data}

Only limited data are available for the heat capacity of aqueous salt solutions. Perry and Chilton (1973) recommend estimating the heat capacity of aqueous solutions by using a weighted combination of the heat capacities of the solid and water. However, a more complicated approach can be justified, based on correlation of the limited data for two salts similar to those in the tank waste solution. Table 10.2 shows correlations for the heat capacities of aqueous solutions of $\mathrm{Na}_{2} \mathrm{CO}_{3}$ and $\mathrm{KNO}_{3}$. 
Table 10.2. Heat capacity correlations (J/kg K) for single-component aqueous solutions. The correlations all use the template in Equation (10.3).

\begin{tabular}{|c|c|c|c|c|c|c|}
\hline Species & $a_{0}$ & $a_{1}$ & $a_{3}$ & $a_{8} * 10^{3}$ & $\begin{array}{c}\text { Max. error } \\
\text { (J/kg K) }\end{array}$ & $\mathbf{R}^{2}$ \\
\hline $\mathrm{Na}_{2} \mathrm{CO}_{3}$ & 3458 & -23.96 & 2.192 & -0.195 & $100(2.7 \%)$ & 1.00 \\
\hline $\mathrm{KNO}_{3}$ & 4088 & -39.18 & 0.270 & 0.2204 & $31(1.4 \%)$ & 1.00 \\
\hline
\end{tabular}

These correlations are applicable over the concentration range from 0 to $100 \%$ solute and the temperature range from 20 to $100^{\circ} \mathrm{C}$. The data for sodium carbonate came from Tables 3-174 and 3-191 of Perry and Chilton (1973); the data for potassium nitrate came from Table 3-174 of Perry and Chilton (1973) and from Table 3.6.5 of Ochs et al. (1981).

\subsubsection{Multi-component}

No data were found for heat capacities of multi-component solutions. Because one of the predominant components of the tank waste is $\mathrm{NaNO}_{3}$, it is recommended the correlation for $\mathrm{KNO}_{3}$ solution in Table 10.2 be used. As shown in Table 10.3, the heat capacities of $\mathrm{NaNO}_{3}$ and $\mathrm{KNO}_{3}$ are roughly equal.

\subsection{Single-phase solid heat capacity}

The solids in tank wastes are primarily composed of sodium nitrate, sodium nitrite, and sodium aluminate (or aluminum hydroxides, such as boehmite and gibbsite).

\subsubsection{Single component}

As an approximation, the solids in Tank 241-SY-101 and other Hanford waste tanks can be treated as containing only a single-component, using one of the major precipitated species as a surrogate for the rest. A single-component template for heat capacity is given in Equation (10.4). 
10.3.1.1 Template for $\mathrm{c}_{\mathrm{ps}}$, the solid heat capacity $(\mathrm{J} / \mathrm{kg} \mathrm{K})$

$$
c_{p s}=a_{0}+a_{3} T
$$

Range: $273-373 \mathrm{~K}$

$$
\text { where } \begin{aligned}
a_{k} & =\text { coefficients obtained by data correlation } \\
\mathrm{T} & =\text { temperature }(\mathrm{K})
\end{aligned}
$$

\subsubsection{Data}

Table 10.3 shows some correlations, taken from Table 3-174 of Perry and Chilton (1973), for the heat capacities of some of the salts in the waste tanks. The reference did not give correlation constants, but did give an uncertainty value to each correlation. The values for sodium aluminate and sodium hydroxide are from Dean (1985).

\begin{tabular}{|c|c|c|c|}
\hline Salt & $a_{0}$ & $a_{3}$ & uncertainty \\
\hline $\mathrm{Na}_{2} \mathrm{CO}_{3}$ & 1141 & & $?$ \\
\hline $\mathrm{NaCl}$ & 772.9 & 0.301 & $2 \%$ \\
\hline $\mathrm{NaNO}_{3}$ & 224 & 2.85 & $5 \%$ \\
\hline $\mathrm{KNO}_{3}$ & 266 & 2.19 & $10 \%$ \\
\hline $\mathrm{NaAlO}_{2}$ & 1090 & & \\
\hline $\mathrm{NaOH}$ & 1490 & & \\
\hline
\end{tabular}

Table 10.3. Heat capacity correlations $(\mathrm{J} / \mathrm{kg} \mathrm{K}$ ) for some single-component salts. The correlations all use the template in Equation (10.4).

\subsubsection{Multi-component}

Given a composition for the solids in the tank waste, and given additional data for the heat capacities of individual solid species, the heat capacities can be combined to obtain an average heat capacity. The equation for the average heat capacity is shown as Equation (10.5). 
10.3.2.1 Template for $c_{p s}$, the solid heat capacity $(\mathrm{J} / \mathrm{kg} \mathrm{K})$

$$
c_{p s}=\sum_{i=1}^{N} C_{i} c_{p i} / 100
$$

Range: all compositions

$$
\text { where } \begin{aligned}
c_{\mathrm{pi}} & =\text { heat capacity of the } \mathrm{i} \text {-th species of } \mathrm{N} \text { solid species }(\mathrm{J} / \mathrm{kg} \mathrm{K}) \\
\mathrm{C}_{\mathrm{i}} & =\text { weight percent of } \mathrm{i} \text {-th solid species }
\end{aligned}
$$

\subsubsection{Data}

The known heat capacities of the solids in tank wastes are given in Section 10.3.1.2. The linear combination of heat capacities in Equation (10.5) is an approximate technique used where no information was found about the non-linear effect of components on the overall density.

\subsection{Liquid-solid (gasless) slurry heat capacity}

The sludge and slurry in the tanks are combinations of liquid, solid, and gas phases. This section characterizes the heat capacity of liquid-solid slurries and sludges, preparatory to Section 10.5, which discusses the more common case of bubbly sludges. The heat capacity of a liquid-solid slurry is described by Equation (10.6).

\subsubsection{Template for $\mathrm{c}_{\mathrm{pm}}$, the gasless slurry heat capacity (J/kg K)}

$$
c_{p m}=\frac{\varepsilon_{c} \rho_{c} c_{p c}+\left(1-\varepsilon_{c}\right) \rho_{s} c_{p s}}{\varepsilon_{c} \rho_{c}+\left(1-\varepsilon_{c}\right) \rho_{s}}
$$

Range: all values of porosity

$$
\begin{aligned}
& \text { where } \begin{array}{ll}
\rho_{c} & =\text { density of the liquid }\left(\mathrm{kg} / \mathrm{m}^{3}\right) \\
\varepsilon_{\mathrm{c}} & =\text { the porosity (volume fraction of sludge not occupied by solid) } \\
c_{\mathrm{pc}} & =\text { heat capacity of the liquid }(\mathrm{J} / \mathrm{kg} \mathrm{K}) \\
\rho_{\mathrm{s}} & =\text { density of the solid }\left(\mathrm{kg} / \mathrm{m}^{3}\right) \\
c_{\mathrm{ps}} & =\text { heat capacity of the solid }(\mathrm{J} / \mathrm{kg} \mathrm{K})
\end{array}
\end{aligned}
$$




\subsubsection{Data}

See Section 4.4 .2 for a further discussion of the slurry and sludge porosity and density, which are used as variables in Equation (10.6).

\subsection{Liquid-solid-gas (bubbly) slurry heat capacity}

The templates given in Equation (4.7), for combining liquid and solid densities, are also appropriate for combining the solution and solid heat capacities to find the heat capacity of gasless sludge and slurry. These templates, as adapted for heat capacity calculation, are shown in Equations (10.7) and (10.8).

\subsubsection{Template for $c_{\mathrm{pb}}$, the bubbly slurry heat capacity (J/kg K)}

$$
c_{p b}=\frac{\left(\varepsilon_{c}-\varepsilon_{d}\right) \rho_{c} c_{p c}+\left(1-\varepsilon_{c}\right) \rho_{s} c_{p s}}{\left(\varepsilon_{c}-\varepsilon_{d}\right) \rho_{c}+\left(1-\varepsilon_{c}\right) \rho_{s}}
$$

Range: for dendritic bubbles; $\varepsilon_{d} \leq \varepsilon_{c}$

$$
c_{p b}=\left(1-\varepsilon_{d}\right) c_{p m}
$$

Range: for particle-displacing bubbles

$$
\begin{aligned}
& \text { where } \begin{array}{ll}
\varepsilon_{\mathrm{d}} & =\text { volume fraction of gas (void fraction) } \\
\varepsilon_{\mathrm{c}} & =\text { the porosity (volume fraction of sludge not occupied by solid) } \\
\rho_{\mathrm{c}} & =\text { density of the liquid }\left(\mathrm{kg} / \mathrm{m}^{3}\right) \\
c_{\mathrm{pc}} & =\text { heat capacity of the liquid }(\mathrm{J} / \mathrm{kg} \mathrm{K}) \\
\rho_{\mathrm{s}} & =\text { density of the solid }\left(\mathrm{kg} / \mathrm{m}^{3}\right) \\
c_{\mathrm{ps}} & =\text { heat capacity of the solid }(\mathrm{J} / \mathrm{kg} \mathrm{K}) \\
c_{\mathrm{pm}} & =\text { heat capacity of the gasless slurry }(\mathrm{J} / \mathrm{kg} \mathrm{K})
\end{array}
\end{aligned}
$$


10.5.2 Data

See Section 4.5.2 for a further discussion of dendritic and particle-displacing bubbles and their effects on sludge properties.

10.8 


\subsection{Thermal Conductivity}

Heat, as well as gas, is generated in the settled solids at the bottom of Hanford waste tanks. The thermal conductivity of the waste sludge is one of the parameters that controls the temperature profile in the sludge, which in turn affects the gas generation rate, the sludge viscosity, and the sludge density (hence, the timing and size of gas release events). Because the sludge is a three-phase mixture of liquid, particles, and gas bubbles, simple linear interpolation between the conductivities of the constituents can be inaccurate. No thermal conductivity estimation methods for three-phase mixtures appear in the literature, but a method for two-phase mixtures has been adapted for three-phase situations.

This chapter begins by providing correlations and other submodels for the thermal conductivity of single phases, the gas in the bubbles (Section 11.1), the solution liquid (Section 11.2), and the solids (Section 11.3). Section 11.4 specifies a submodel for the gasless slurry thermal conductivity, and Section 11.5 discusses the bubbly slurry thermal conductivity.

\subsection{Single-phase gas thermal conductivity}

The gas in the tank sludge is believed to be present in the form of small dendritic or spherical bubbles,. As such, the gas thermal conductivity plays a significant role in the effective thermal conductivity of the sludge and, consequently, of the overall tank thermal behavior.

\subsubsection{Single component}

Because the gas in the tanks is at near-ambient temperatures and pressures, its thermal conductivity can be treated as dependent on temperature, independent of pressure differences. The multi-component waste tank gas can be represented by one of its components, if that species is sufficiently predominant. Equation (11.1) gives a template for single-component gas thermal conductivity. 
11.1.1.1 Template for $k_{i}$, the thermal conductivity of a gaseous species $(W / m K)$

$k_{i}=a_{0}+a_{3} T$

Range: temperature $273-373 \mathrm{~K}$; pressure less than $3 \mathrm{~atm}$

where $a_{k} \quad=$ coefficients found by correlation

$\mathrm{T} \quad=$ temperature $(\mathrm{K})$

\subsubsection{Data}

Table 11.1 contains the thermal conductivity correlation constants for the predominant gases in the waste tank gas. (The gas composition has been described in Section 4.1.1.2.) All of these correlations were within $1 \%$ of the data, which were taken from p. E-2 of CRC (1975).

Table 11.1. Thermal conductivity correlations $(\mathrm{W} / \mathrm{m} \mathrm{K})$ for gases present in the waste tank gas. The correlations all use the template in Equation (11.1).

\begin{tabular}{|c|c|c|}
\hline Gas & $a_{0} * 103$ & $a_{3} * 10^{3}$ \\
\hline $\mathrm{H}_{2}$ & 82.2 & 0.294 \\
\hline $\mathrm{N}_{2}$ & 11.9 & 0.0400 \\
\hline $\mathrm{N}_{2} \mathrm{O}$ & 1.28 & 0.0457 \\
\hline $\mathrm{NH}_{3}$ & 5.10 & 0.0554 \\
\hline
\end{tabular}

\subsubsection{Multi-component}

Alternately, the gas can be represented as a multi-component mixture in which the volume fraction and molecular weight of each species are used to calculate a mixture average thermal conductivity as in Equation (11.2). 


\subsubsection{Template for $k_{d}$, the gas thermal conductivity (W/m K)}

$$
k_{d}=\frac{\sum_{i=1}^{N} x_{i} k_{j} W_{i}^{1 / 3}}{\sum_{i=1}^{N} x_{j} W_{i}^{1 / 3}}
$$

Range: temperature $273-373 \mathrm{~K}$; pressure less than 3 atm

$$
\text { where } \begin{aligned}
k_{i} & =\text { thermal conductivity of each gas species } \mathrm{i} \text { in the mixture }(\mathrm{W} / \mathrm{m} \mathrm{K}) \\
\mathrm{x}_{\mathrm{i}} & =\text { mole or volume fraction of the } \mathrm{i} \text {-th species of } \mathrm{N} \text { gaseous species } \\
\mathrm{W}_{\mathrm{i}} & =\text { molecular weight of the } \mathrm{i} \text {-th species }(\mathrm{kg} / \mathrm{gmol})
\end{aligned} .
$$

\subsubsection{Data}

The molecular weights and composition data for the gas in Tank 241-SY-101 wastes are the same as those given in Section 4.1.1.2. The template was taken from equation (3-100) in Perry and Chilton (1973), who stated that the equation gave maximum errors of less than $10 \%$ for 19 lowpressure binary gas mixtures in the temperature range $273-373 \mathrm{~K}$. Other, more complicated equations for the thermal conductivity of gas mixtures are available, but are only slightly more accurate than Equation (11.2) (Perry and Chilton 1973). One such equation may be found in Section 8.3 of Bird et al. (1960).

\subsection{Single-phase liquid thermal conductivity}

The waste tank supernatant solution contains predominantly $\mathrm{NaNO}_{3}$ and $\mathrm{NaNO}_{2}$, with much smaller quantities of other nitrates and nitrate salts.

\subsubsection{Single component}

In a case where one salt is present in a solution in much greater quantities than others, it is plausible to treat the solution as single-component. In the case of tank waste solutions, this approach [shown in Equation (11.3)] is approximate, but necessary, because of the scarcity of salt solution thermal conductivity data. 
11.2.1.1 Template for $k_{c}$, the liquid thermal conductivity (W/m K)

$$
k_{c}=a_{0}+a_{1} C+a_{2} C^{2}+a_{3} T+a_{5} C T
$$

Range: temperature 273-373 K; concentration 0-100 wt\%

$$
\text { where } \begin{aligned}
a_{k} & =\text { coefficients found by correlation } \\
C & =\text { weight percent solute } \\
T & =\text { temperature }(K)
\end{aligned}
$$

\subsubsection{Data}

No information was found for the thermal conductivity of any nitrate or nitrite aqueous

\begin{tabular}{|c|c|c|c|c|c|}
\hline Liquid & $a_{0}$ & $a_{1}$ & $a_{2} * 10^{3}$ & $a_{3} * 10^{3}$ & $a_{5} * 106$ \\
\hline $\mathrm{NaCl}(\mathrm{aq})$ & 0.2961 & -0.02177 & 1.096 & 1.190 & -7.831 \\
\hline Water & 0.2539 & & & 1.184 & \\
\hline
\end{tabular}
solutions. Table 11.2 gives the thermal conductivities of aqueous $\mathrm{NaCl}$ solutions and water.

\begin{tabular}{|c|c|c|c|c|}
\hline Liquid & $\begin{array}{l}\text { Temp. } \\
\text { range } \\
(\mathrm{K})\end{array}$ & $\begin{array}{l}\text { Conc. } \\
\text { range } \\
\text { (wt \%) }\end{array}$ & $\begin{array}{c}\text { Corre- } \\
\text { lation } \\
\mathbf{r}^{2}\end{array}$ & $\begin{array}{l}\text { Max. error } \\
(\mathrm{W} / \mathrm{m} \mathrm{K})\end{array}$ \\
\hline $\mathrm{NaCl}$ (aq) & $273-373$ & $0-100$ & 1.00 & $0.05(8.3 \%)$ \\
\hline Water & $293-363$ & 0 & 0.98 & $0.0054 \quad(0.9 \%)$ \\
\hline
\end{tabular}

Table 11.2. Thermal conductivity correlations $(\mathrm{W} / \mathrm{m} \mathrm{K})$ for sodium chloride solution and water. The correlations all use the template in Equation (11.3).

Data in Table 3.3.6 of Ochs et al. (1981) covered the range from 5-20 wt\% $\mathrm{NaCl}$ solutions, and the data for pure water and for solid crystalline $\mathrm{NaCl}$ came from pages $\mathrm{E}-11$ and $\mathrm{E}-4$ of CRC (1975).

Thermal conductivity data are lacking for $\mathrm{NaNO}_{3}$ and $\mathrm{NaNO}_{2}$ solutions. A suggested approximation is to use the difference between the thermal conductivities of $\mathrm{NaCl}$ solution and water, 
estimated from Equation (11.3) and Table 11.2, and scale that by the thermal conductivity of the solid solute. (Values for the thermal conductivity of some solid salts can be found in Section 11.3.1.2.)

For example, the thermal conductivity of solid $\mathrm{NaCl}$ is $9.2 \mathrm{~W} / \mathrm{m} \mathrm{K}$ at $0^{\circ} \mathrm{C}$; that of solid $\mathrm{KNO}_{3}$ is $2.1 \mathrm{~W} / \mathrm{m} \mathrm{K}$; that of water is $0.561 \mathrm{~W} / \mathrm{m} \mathrm{K}$. As an approximation, the thermal conductivity of potassium nitrate solution could be calculated as

$$
\mathrm{k}_{\mathrm{KNO3,aq}}=\left(\mathrm{k}_{\mathrm{NaCl}, \mathrm{aq}}-\mathrm{k}_{\mathrm{water}}\right) *(2.1-0.561) /(9.2-0.561)+\mathrm{k}_{\mathrm{water}}
$$

This technique assumes the thermal conductivity of all salt solutions has the same functional variation with temperature and concentration as does $\mathrm{NaCl}$. Under the circumstances, this approximation is unavoidable.

\subsubsection{Multi-component}

No data were found for the thermal conductivities of multi-component solutions. Because one of the predominant components of the tank waste is $\mathrm{NaNO}_{3}$, it is recommended the scaling method described in Section 11.2.1.2 be used to approximate the thermal conductivity of a $\mathrm{KNO}_{3}$ solution. This, in turn, can be taken as a fair approximation of thermal conductivity for an $\mathrm{NaNO}_{3}$ solution.

\subsection{Single-phase solid thermal conductivity}

The tank waste solids are primarily composed of sodium nitrate, sodium nitrite, and sodium aluminate (or aluminum hydroxides, such as boehmite and gibbsite). Sodium chloride, carbonate, citrate, sulfate, and other salts are present only in small quantity.

\subsubsection{Single component}

As an approximation, the solids in the Hanford waste tanks can be treated as containing only a single component, using one of the major precipitated species as a surrogate for the rest. 


\subsubsection{Template for $k_{s}$, the solid thermal conductivity (W/m K)}

$$
\mathrm{k}_{\mathrm{s}}=\mathrm{a}_{0}
$$

Range: temperature $273-373 \mathrm{~K}$

where $k_{s} \quad=a$ constant (refer to Table 11.3)

\subsubsection{Data}

The thermal conductivities of some dielectric salts are given by the CRC Handbook (1975, p. E-4) and repeated in Table 11.3. The highest temperature at which the reference provides values is $0^{\circ} \mathrm{C}$, or $273 \mathrm{~K}$. Hence, the temperature dependence of the thermal conductivity is not known.

Table 11.3. Thermal conductivities (W/m K) of some solid salts.

\begin{tabular}{lll} 
Salt & & $\mathrm{k}_{s}(\mathrm{~W} / \mathrm{m} \mathrm{K})$ \\
\cline { 1 - 1 } $\mathrm{NaCl}$ & & 9.2 \\
$\mathrm{KCl}$ & 9.2 \\
$\mathrm{KNO}_{3}$ & 2.1 \\
$\mathrm{KAl}\left(\mathrm{SO}_{4}\right)_{2}$ & & 2.1
\end{tabular}

Based on the data in CRC (1975), it appears that many univalent salts that have compound anions (such as sulfates and chromates) possess thermal conductivities in a range between 1.7 and $2.5 \mathrm{~W} / \mathrm{m} \mathrm{K}$. Thus, the value given for $\mathrm{KNO}_{3}$ may be representative (within $20 \%$ or so) of all the solids in the tank wastes.

\subsubsection{Multi-component}

(no available data)

\subsection{Liquid-solid (gasless) slurry thermal conductivity}

The sludges and slurries in the waste tanks are composed of solutions whose thermal conductivities range from 0.6 to $1.0 \mathrm{~W} / \mathrm{m} \mathrm{K}$, according to the calculation method described in Section 11.2.1, mixed with solids whose thermal conductivity is thought to be $2.1 \mathrm{~W} / \mathrm{m} \mathrm{K}$. In this case, the

\section{6}


difference between the liquid and solid thermal conductivities is small enough to make it possible to use a simple volume fraction weighting to calculate the thermal conductivity of the sludge. However, this simple method would be severely inaccurate in the general case. To maintain generality, a more complex and accurate method is described here.

The most common analytical approach to estimating two-phase thermal (or electrical) conductivities is to take a weighted average, not of the two phases, but of two easily represented mixtures of phases (Hadley 1986; Nakamura 1982; Nakamura 1984; Nan and Smith 1991; Nimick and Leith 1992). Hadley (1986) found that any mixture expression that depends only on fluid volume fraction $\phi$ and the ratio of conductivities $k_{m} / k_{f}=\kappa$ can be represented by the general relations in Equations (11.4) and (11.5).

$$
\begin{aligned}
& \frac{k}{k_{f}}=\frac{\phi f+k(1-\phi f)}{1-\phi(1-f)+\kappa \phi(1-f)} \\
& \kappa=\frac{k_{h}}{k_{f}}
\end{aligned}
$$

$$
\begin{aligned}
& \text { where } \mathbf{k}=\text { effective thermal conductivity of the mixture } \\
& \mathrm{k}_{\mathrm{f}} \quad=\text { thermal conductivity of fluid (that is, the lower-conductivity phase) } \\
& \mathrm{k}_{\mathrm{h}} \quad=\text { thermal conductivity of matrix (higher-conductivity phase) } \\
& \phi \quad=\text { volume fraction of the lower-conductivity phase, or porosity } \\
& \text { f }=\text { a function of } \phi \text { and } \kappa \text {, to be determined from data; it depends on the }
\end{aligned}
$$

This generalized mixture model was expected to be applicable for mixtures of any concentrations, including the dilute or concentrated ranges covered by Maxwell's relations, Equations (11.6) and (11.7):

$$
\begin{array}{ll}
\frac{k}{k_{f}}=\frac{2 \phi+\kappa(3-2 \phi)}{3-\phi+\kappa \phi} & \text { for high } \phi \text { range (dilute) } \\
\frac{k}{k_{f}}=\frac{2 \kappa^{2}(1-\phi)+(1+2 \phi) \kappa}{(2+\phi) \kappa+1-\phi} & \text { for low } \phi \text { range (concentrated) }
\end{array}
$$


These two Maxwell formulas [cited by Hadley (1986)] are the most stringent upper and lower bounds for the thermal conductivity of homogeneous, isotropic, two-phase mixtures. It follows the function $f(\phi, \kappa)$ lies between the bounds that correspond to the Maxwell formulas, as shown in Equation (11.8):

$$
\frac{2}{3}<\mathrm{f}<\frac{2 \mathrm{~K}}{2 \mathrm{k}+1}
$$

Hadley (1986) showed the function $f$ would have the limiting value of $2 / 3$ at $\phi=1$ and increase monotonically with $(1-\phi)$ for a suspension of non-touching particles. To include the effect of particle contact, he introduced another system-dependent parameter, $\alpha$. This parameter is used to weight the contribution of the general mixture [Equation (11.4)] that represents the non-contacting particles, and the contribution of the concentrated mixture [Equation (11.7)] that represents the incontact particles. Hadley used Equation (11.9) as the general form for the effective thermal conductivity of a mixture:

$$
\frac{k}{k_{f}}=(1-\alpha) \frac{\phi f+\kappa(1-\phi f)}{1-\phi(1-f)+\kappa \phi(1-f)}+\alpha \frac{2 \kappa^{2}(1-\phi)+(1+2 \phi) \kappa}{(2+\phi) \kappa+1-\phi}
$$

Here $\alpha$ and $f$ must be determined from data. Quoting Hadley (p. 915, Hadley 1986): “The parameter $f$ is expected to be approximately constant for a contiguous solid, whereas $\alpha$ will depend strongly on what may be called the 'degree of consolidation'. For granular systems $\alpha$ is small and the first term in (7.6) is dominant for moderate values of $\kappa$. For consolidated materials, however, $\alpha$ is moderate and the second term may be dominant over most of the range of $\kappa . "$

Hadley also showed it was theoretically possible to describe a three-phase system by treating it as two two-phase systems, a result that should be directly applicable to the three-phase bubbly slurry in the Hanford waste tanks. This approach is followed by separating the slurry into the solid-liquid mixture [Equation (11.10) and Section 11.4.2] and then considering the mixture of the solid-liquid slurry with the gas [Equations (11.13) and (11.14) and Section 11.5]. 


\subsubsection{Template for $k_{m}$, the gasless slurry thermal conductivity (W/m K)}

$$
\frac{k_{m}}{k_{c}}=\left(1-\alpha_{m}\right) \frac{\varepsilon_{c} f_{m}+\kappa_{m}\left(1-\varepsilon_{c} f_{m}\right)}{1-\varepsilon_{c}\left(1-f_{m}\right)+\kappa_{m} \varepsilon_{c}\left(1-f_{m}\right)}+\alpha_{m} \frac{2 \kappa_{m}^{2}\left(1-\varepsilon_{c}\right)+\left(1+2 \varepsilon_{c}\right) \kappa_{m}}{\left(2+\varepsilon_{c}\right) \kappa_{m}+1-\varepsilon_{c}}
$$

Range: $\varepsilon_{\mathrm{c}}$ from 0 to $1, \kappa_{\mathrm{m}}=9.1 ; \varepsilon_{\mathrm{d}}$ from 0 to 0.6

$$
\begin{aligned}
& \text { where } k_{m} \quad=\text { thermal conductivity of the gasless sludge }(\mathrm{W} / \mathrm{m} \mathrm{K}) \\
& k_{\mathfrak{c}} \quad=\text { thermal conductivity of the liquid, for sludge; of the less-conductive } \\
& \text { material, for the general case }(\mathrm{W} / \mathrm{m} \mathrm{K}) \\
& \alpha_{\mathrm{m}}=\text { the consolidation fitting parameter for the gasless sludge (from data) } \\
& f_{m} \quad=\text { the fitting function for the gasless sludge (from data) } \\
& \varepsilon_{\mathrm{c}} \quad=\text { the volume fraction of liquid in the gasless sludge } \\
& \kappa_{\mathrm{m}}=\mathrm{k}_{\mathrm{s}} / \mathrm{k}_{\mathrm{c}} \text { (solid thermal conductivity/liquid) for the sludge; in general, } \\
& \text { the ratio }(\geq 1) \text { of the larger to the smaller thermal conductivity }
\end{aligned}
$$

\subsubsection{Data}

Hadley (1986) used three-phase and two-phase experimental data obtained by himself and others to derive and test values of $f$ and $\alpha$. His data base included consolidated discs of hot-pressed brass and stainless-steel powder, with zero voids; consolidated discs of cold-pressed brass and stainless-steel powder, with void fractions up to 31 vol\%; and mixtures of loose brass and stainlesssteel powder with void fraction between 40 and 60 vol\%. The fluids in the voids were vacuum, air, or water. The sizes of the particles in the powder were not stated.

Hadley compared the thermal conductivity of each metal-metal mixture to that of brass, at the same void fraction $\varepsilon_{\mathrm{d}}$, to find a correlation for the effective thermal conductivity of a mixed metal matrix. This correlation was described by equation (11.10), using the functions in Equations (11.11) and (11.12). 
$\alpha_{\mathrm{m}}=0$

$$
f_{m}=\min \left\{\begin{array}{l}
\frac{2 \kappa_{m}}{2 \kappa_{m}+1} \\
\frac{2}{3}\left(\frac{\left(\kappa_{m}-1\right)}{0.3\left(2 \kappa_{m}+1\right)} \varepsilon_{d}+1\right)
\end{array}\right\}
$$

where $\varepsilon_{d}=$ the volume fraction of gas in the sludge; $\varepsilon_{d}=0$ for gasless sludge

The function $f_{m}$ was selected to make $f_{m}$ equal to the dilute Maxwell limit at $\varepsilon_{d}=0$ and the concentrated Maxwell limit at $\varepsilon_{\mathrm{d}} \geq 0.3$ (as appeared appropriate from the data). The zero value for $\alpha_{\mathrm{m}}$ is derived from the data. For completely gasless sludge, $f_{m}=2 / 3$ and the effective thermal conductivity of the gasless solid-liquid sludge matrix follows Maxwell's dilute relation [Equation (11.6)] at all steel concentrations, dilute or not. It is interesting to note the thermal conductivity of the matrix varies with void fraction; such a variation would be expected for the conductivity of the porous material as a whole, but not necessarily for that of the matrix itself.

Although the metal-metal matrix used by Hadley (1986) seems very different from the solidliquid matrix in the sludge, the morphologies of the two systems are probably rather similar. The . pressing together of angular particles of steel and (relatively) soft brass is expected to produce irregular shapes and wide contact areas rather like those of the solid sludge particles surrounded and bridged by solution. Hadley's data only included one value of $\kappa_{\mathrm{m}}$, equal to 9.1 for steel and brass. This value of $\kappa_{\mathrm{m}}$ is of the same order of magnitude as that for the solids and liquid in sludge, a value of 2 to 3 .

The Hadley submodel's error in predicting the effective matrix thermal conductivity at zero void fraction was less than $10 \%$.

\subsection{Liquid-solid-gas (bubbly) slurry thermal conductivity}

In modeling a bubbly slurry; the bubble morphology must be taken into account. A slurry or sludge containing dendritic bubbles can be expected to resemble the pressed metal discs on which Hadley (1986) experimented, and to be describable by Hadley's submodel. A slurry or sludge 
containing spherical bubbles should more closely resemble Maxwell's concentrated submodel [Equation (11.7)], which assumed spheres of low-conductivity material embedded in a highconductivity matrix. Equations (11.13) and (11.14) follow this rationale.

11.5.1 Template for $k_{b}$, the bubbly slurry thermal conductivity (W/m K)

$$
\frac{k_{b}}{k_{d}}=(1-\alpha) \frac{\varepsilon_{d} f+\kappa\left(1-\varepsilon_{d} f\right)}{1-\varepsilon_{d}(1-f)+\kappa \varepsilon_{d}(1-f)}+\alpha \frac{2 \kappa^{2}\left(1-\varepsilon_{d}\right)+\left(1+2 \varepsilon_{d}\right) \kappa}{\left(2+\varepsilon_{d}\right) \kappa+1-\varepsilon_{d}}
$$

Range: for dendritic bubbles (those which displace liquid but not particles); $\varepsilon_{\mathrm{d}} \leq \varepsilon_{\mathrm{c}}$

$$
\frac{k_{b}}{k_{d}}=\frac{2 \kappa^{2}\left(1-\varepsilon_{d}\right)+\left(1+2 \varepsilon_{d}\right) \kappa}{\left(2+\varepsilon_{d}\right) \kappa+1-\varepsilon_{d}}
$$

Range: for roughly spherical bubbles (which displace both liquid and particles)

$$
\begin{aligned}
& \text { where } k_{b}=\text { thermal conductivity of the bubbly sludge }(\mathrm{W} / \mathrm{m} \mathrm{K}) \\
& k_{d} \text {. = thermal conductivity of the gas, for bubbly sludge; of the less- } \\
& \text { conductive material, for the general case (W/m K) } \\
& \alpha=\text { the consolidation fitting parameter for the bubbly sludge (from data) } \\
& \text { f = the fitting function for the bubbly sludge (from data) } \\
& \varepsilon_{\mathrm{d}} \quad=\text { the volume fraction of gas in the bubbly sludge } \\
& \kappa=\quad=k_{m} / k_{d} \text { (gasless sludge thermal conductivity/gas) for the sludge; } \\
& \text { in general, the ratio }(\geq 1) \text { of the larger to the smaller thermal conductivity }
\end{aligned}
$$

\subsubsection{Data}

Hadley (1986) used the effective thermal conductivity of the metals [calculated with Equations (11.10) through (11.12)] as the matrix conductivity and solved again to find the overall effective thermal conductivity, including the fluid (vacuum, air, or water). For this system, Hadley found that $\alpha$ was a well-defined function of $\varepsilon_{\mathrm{d}}$ for both consolidated solids (pressed powder discs) and loose powders. The algorithms [Equations (11.13), (11.15), and (11.16)] derived by Hadley (1986) described his data, for the fluids air and water, with an average error of $16 \%$ and a maximum error of $67 \%$. 


$$
\begin{aligned}
& \alpha=\left\{\begin{array}{ll}
\mathrm{e}^{-11.45 \varepsilon_{\mathrm{d}}} & \varepsilon_{\mathrm{d}}<0.08 \\
0.706 \mathrm{e}^{-7.103 \varepsilon_{\mathrm{d}}} & 0.08 \leq \varepsilon_{\mathrm{d}}<0.3 \\
8.116 \mathrm{e}^{-15.52 \varepsilon_{\mathrm{d}}} & 0.3 \leq \varepsilon_{\mathrm{d}}
\end{array}\right\} \\
& \mathrm{f}=0.86
\end{aligned}
$$

Hadley (1986) also compared his model predictions with other researchers' two-phase data. Most of Hadley's predictions fell within $20 \%$ of literature data. The largest disparity between Hadley's predictions and others' observations occurred with experiments involving fine powders in low-pressure gases. In these cases, molecular conduction in the small pores would have caused strong variation in $\mathbf{k}_{\mathrm{f}}$ and a corresponding departure from Hadley's submodel, which assumed constant fluid thermal conductivity. Hadley compared his model to the two-phase experimental data and theoretical predictions of Nozad et al. (1985a, b) and found substantial agreement. Nimick and Leith (1992) compared their own model to Hadley's for granular silica powder in air and found that the estimate produced by Hadley's model was less than $10 \%$ off.

The tank sludge is expected to have a void fraction in the range between 0 and 20 vol\% and $\kappa$ between 9 and 30. (From Section 11.4, the gasless sludge thermal conductivity is between 0.6 and $2.1 \mathrm{~W} / \mathrm{m} \mathrm{K}$, and from Section 11.1, the gas thermal conductivity is about $0.068 \mathrm{~W} / \mathrm{m} \mathrm{K}$.) In this range of $K$, the Hadley submodel consistently predicts within $20 \%$ of the data. (However, Hadley collected only a few data points in this range.)

Other thermal conductivity models were checked against Hadley's two-phase and three-phase data and found to perform less well. Several combinations of linear interpolation (using volume fractions), Maxwell's lower (dilute) formula, and Maxwell's higher (concentrated) formula were found to give much higher errors in the predicted effective thermal conductivity than Hadley's submodel. A unit-cell thermal conductivity model originally developed by Zehner and Bauer and described by Tsotsas and Martin (1987) was also tested against Hadley's data and found less satisfactory. This last submodel may be more appropriate for typical process packed beds with particle sizes of $1 \mathrm{~mm}$ and greater; it has been verified against data for such packed beds by Tsotsas and Martin (1987). 


\subsection{Bubble Rise}

The behavior of the gas bubbles in the waste tank sludge and supernatant is of central importance in modeling the sludge turnovers and gas releases. The TEMPEST code describes bubbly fluid, or suspensions, with a pseudofluid submodel that considers only the cell average physical quantities.

A more detailed model is discussed in this section. The gas (dispersed) and liquid (continuous) phases are separated for the purpose of deriving fundamental mass and momentum balances. This separation permits a definition of momentum transfer between the dispersed and continuous phases. The drag and virtual mass forces provide coupling between the phases. Equations are supplied to allow the calculation of both forces for bubble swarms as well as single bubbles. The effect of the presence of particles on the bubble drag force and rise velocity can also be included.

This discussion of bubble rise models is separated into four sections. Sections 12.1 and 12.2 derive the mass balances and momentum balances for the dispersed and continuous phases. These equations support treating the gas and liquid as distinct interacting phases. They are needed to describe the context into which the correlation models fit, although TEMPEST does not directly use these multi-field balances. (TEMPEST simplifies calculations and increases the computational efficiency by using the pseudofluid approach rather than a multi-field approach.) Section 12.3 provides correlation models for bubble and particle drag coefficients, and Section 12.4 gives experimentally-based equations for the virtual mass force on bubbles.

\subsection{Mass balance}

The mass balances for a dispersed phase, and the continuous phase in which it is found, can be derived for the general multi-field case. For the case of gas bubbles in liquid, component $d$ represents the dispersed gas phase and component $c$ the continuous liquid phase. For the case of suspensions, component $d$ represents the particles. This derivation ignores the dispersed species dissolved in the liquid and the liquid incorporated into the dispersed phase. It assumes only one chemical species is present in each field, no reaction produces liquid, and the mass lost to gas must be balanced.

The continuity equations for the gas and liquid phases are given as Equations (12.1) and (12.2). 


$$
\begin{aligned}
& \frac{\partial}{\partial t}\left(\rho_{d} \varepsilon_{d}\right)+\nabla \cdot \rho_{d} \varepsilon_{d} u_{d}=R \\
& \frac{\partial}{\partial t}\left(\rho_{c} \varepsilon_{c}\right)+\nabla \bullet \rho_{c} \varepsilon_{c} u_{c}=-R
\end{aligned}
$$

where $\rho_{c}=$ density of liquid phase

$\rho_{\mathrm{d}} \quad=$ density of gas phase

$\varepsilon_{\mathrm{c}} \quad=$ volume fraction of liquid phase

$\varepsilon_{\mathrm{d}} \quad=$ volume fraction of gas phase

$\mathbf{u}_{\mathrm{c}} \quad=$ velocity of liquid phase

$\mathbf{u}_{\mathbf{d}} \quad=$ velocity of gas phase

$\mathrm{R} \quad=$ rate of generation of gas mass per unit volume of liquid plus gas

Analogously, it is possible to derive conservation equations for the number density $N_{d}$ of the dispersed phase (number of bubbles per unit volume). If the gas bubble distribution is monodisperse, only one number density variable need be considered. The complete number density conservation equation for the gas phase is given as Equation (12.3).

$$
\frac{\partial N_{d}}{\partial t}+\nabla \cdot N_{d} u_{d}=R_{N}
$$

where $\mathbf{R}_{\mathbf{N}}=$ the number rate of generation of bubbles per volume

The equivalent diameter $D_{d}$ of the bubbles is derived from the two transported quantities, the gas void fraction and the number density, as follows in Equation (12.4):

$$
\mathrm{D}_{d}=\left(\frac{6 \varepsilon_{d}}{\pi \mathrm{N}_{\mathrm{d}}}\right)^{1 / 3}
$$

The number density production rate and the gas production rate can be related to each other, as in Equation (12.5): 


$$
R_{N}=\frac{6 R}{\pi \rho_{d} D_{d}^{3}}
$$

These forms of the transport equations allow for change in average bubble size as the bubbles rise. These equations can be extended to cover the case of a polydisperse bubble distribution, but the bubble population must be subdivided into bins. Each bin contains a part of the population that has approximately equivalent rise velocity and other transport behavior. A separate $\varepsilon_{d}, N_{d}, u_{d}$, and $R$ must be specified for each bin, and the (potentially varying) equivalent diameter for the bin is calculated from $\varepsilon_{d}$ and $N_{d}$. Bubble mass may have to be accounted for in one bin instead of another, if the range of transport behavior within a bin becomes too great. In addition, a pseudo-reaction term has to be incorporated to include coalescence and breakup, which transport gas and number density between bins. Each of the mass conservation equations for a polydisperse gas phase is of the form shown in Equation (12.6).

$$
\frac{\partial}{\partial t}\left(\rho_{d} \varepsilon_{d j}\right)+\nabla \cdot \rho_{d} \varepsilon_{d j} u_{d j}=R_{j}+\sum_{n=1}^{N}\left(r_{n, j}-r_{j, n}\right)
$$

Here $\varepsilon_{d j}$ is the volume fraction of bubbles in bin $j, R_{j}$ is the rate of gas generation that goes into $\mathrm{j}$-bin bubbles, and $\mathrm{u}_{\mathrm{dj}}$ is their velocity. On the right side of the equation, the summation contains the rates of generation and removal of $j$-bin bubbles: $r_{n, j}$ is the gas rate per unit volume that goes into formation of $j$-bin bubbles from coalescence or breakup of $n$-bin bubbles, and $r_{j, n}$ is the gas rate per unit volume of formation of $\mathrm{n}$-bin bubbles from $\mathrm{j}$-bin bubbles. $\mathrm{N}$ different bins of bubbles are considered.

The corresponding number density conservation equation is shown as Equation (12.7).

$$
\frac{\partial N_{d j}}{\partial t}+\nabla \cdot N_{d j} \mathbf{u}_{d j}=R_{N j}+\frac{6}{\pi \rho_{d} D_{d}^{3}} \sum_{n=1}^{N}\left(r_{n, j}-r_{j, n}\right)
$$

where $N_{d j}=$ number density of $\mathrm{j}$-bin bubbles

$R_{\mathrm{Nj}} \quad=$ number rate of creation of $\mathrm{j}$-bin bubbles per unit volume, resulting from gas generation (not from breakup or coalescence of other bubbles) 
Note that $\mathrm{R}_{\mathrm{Nj}}$ is equal to zero except for the bin corresponding to the smallest bubble size; reaction removes or creates only the smallest bubbles.

To maintain the overall gas mass balance, Equations (12.8) and (12.9) must hold.

$$
\begin{aligned}
& \varepsilon_{d}=\sum_{j=1}^{N} \varepsilon_{d j} \\
& \sum_{j=1}^{N} \sum_{n=1}^{N}\left(r_{n, j}-r_{j, n}\right)=0
\end{aligned}
$$

\subsection{Momentum balance}

The multi-field momentum balance over the control volume is, for the $\mathrm{j}$ bin of the dispersed (gas) phase,

$$
\begin{aligned}
& \frac{\partial}{\partial t}\left(\rho_{d} \varepsilon_{d j} u_{d j}\right)+\nabla \cdot \rho_{d} \varepsilon_{d j} u_{d j} u_{d j}=-\nabla \varepsilon_{d j} P+\rho_{d} \varepsilon_{d j} g-\frac{\pi}{8} C_{d j} N_{d j} D_{d j}^{2} \rho_{c}\left|u_{d j}-u_{c}\right|\left(u_{d j}-u_{c}\right) \\
& -\frac{\pi}{6} C_{v m j} N_{d j} D_{d j}^{3} \rho_{c} a_{R j}+R_{j} u_{c}+\sum_{n=1}^{N}\left(r_{n, j} \mathbf{u}_{d n}-r_{j, n} u_{d j}\right)
\end{aligned}
$$

$$
\text { where } \begin{array}{ll}
\mathrm{P} & =\text { pressure } \\
\mathrm{g} & =\text { acceleration of gravity } \\
\mathrm{A}_{\mathrm{dj}} & =\text { the cross-sectional area of a j-bin bubble } \\
\mathrm{C}_{\mathrm{dj}}, \quad=\text { drag coefficient between bubble and liquid }
\end{array}
$$

The appropriate value for $C_{d}$ is discussed further in Section 12.3. For general applicability, an objective (frame-invariant) version of the virtual mass acceleration $\mathbf{a}_{R}$ should be used. The virtual mass coefficient, $\mathrm{C}_{\mathrm{vm}}$, and the equation for the acceleration are provided in Section 12.4. The liquidupon-gas forces, which are part of the gas-phase momentum equation in Equation (12.10), are the negative of the sum of the gas-upon-liquid forces. Other forces that are present are the Basset force (transient boundary-layer buildup), lift force, and bubble deformation force. These phenomena are neglected in this derivation for lack of applicability in Tank SY-101 circumstances. 
In the gas phase, momentum sources are of two kinds. The mass entering the gas phase from gas generation is assumed to arrive with the same velocity as the liquid phase. The other momentum source arrives with mass from (or goes into) bubbles of other bins, and the associated velocity is that of the originating bubble bin.

The differential equation that can be derived from the momentum and force balance for the liquid phase is given as Equation (12.11).

$$
\begin{aligned}
& \frac{\partial}{\partial t}\left(\rho_{c} \varepsilon_{c} u_{c}\right)+\nabla \bullet \rho_{c} \varepsilon_{c} u_{c} u_{c}=-\nabla \varepsilon_{c} P-\nabla \bullet \varepsilon_{c} \tau+\rho_{c} \varepsilon_{c} g \\
& +\frac{\pi}{8} \rho_{c} \sum_{j=1}^{N} C_{d j} N_{d j} D_{d j}^{2} \mid u_{d j}-u_{c} l\left(u_{d j}-u_{c}\right)+\frac{\pi}{6} \rho_{c} \sum_{j=1}^{N} C_{v m j} N_{d j} D_{d j}^{3} a_{R j}-\sum_{j=1}^{N} R_{j} u_{c} \\
& -\sum_{j=1}^{N} \sum_{n=1}^{N}\left(r_{n, j} \mathbf{u}_{d n}-r_{j, n} u_{d j}\right)
\end{aligned}
$$

where $\tau \quad=$ shear stress (in the $x-y, x-z$, or $y-z$ plane)

The pressure $P$ is assumed to be the same in the gas and liquid phases; that is, the slight excess of gas-phase pressure owing to surface tension is treated as negligible. The shear stress $\tau$ is ignored for the gas phase, as the phase is discontinuous and unable to transmit shear from one side of the control volume to the other.

In practice, the gas-phase momentum is not carried as a separate variable, as in a two-fluid model. Instead, the relative velocity $u_{R j}$ is used, as defined in Equation (12.12).

$$
\mathbf{u}_{\mathrm{Rj}}=\mathbf{u}_{\mathrm{dj}}-\mathbf{u}_{\mathrm{c}}
$$

The equations for the relative phase velocities can be derived by subtracting Equation (12.10), for each bin, from Equation (12.11). 


\subsection{Bubble drag}

This section describes two different submodels of single-bubble rise over all rise regimes from viscous to inertial (Section 12.3.1). These single-bubble rise models can be multiplied by a function of void fraction to get the rise velocity for a bubble swarm (Section 12.3.2). The effects of the particles in the sludge and the non-Newtonian rheology are discussed in Section 12.3.3.

\subsubsection{Single-bubble rise}

The single-bubble rise submodel proposed by Fan and Tsuchiya (1990) has the advantage of using a single explicit equation that is accurate over all bubble rise regimes. The submodel set forth by Wallis (1974) contains several equations, each of which applies only to a single regime; it also sets criteria to define the regimes. Under some conditions, the Wallis model may be less computationally intensive.

\subsubsection{Fan and Tsuchiya submodel for single bubble rise}

The Fan and Tsuchiya (1990) single-bubble submodel provides a single explicit equation that predicts bubble rise velocity within about $20 \%$ over a wide range of bubble size and fluid properties. The average standard deviation was from $-6 \%$ to $+9 \%$ for 20 different Newtonian fluids.

The general equation for the bubble rise velocity [adapted from equation 2.11 in Fan and Tsuchiya (1990, p. 43)] is given in Equation (12.13).

$$
U_{b}=\left[\left(\frac{D_{d}^{2} g\left(\rho_{c}-\rho_{d}\right)}{K_{b} \mu_{c}}\right)^{-n}+\left(\frac{2 c \sigma}{\rho_{c} D_{d}}+\frac{g D_{d}}{2}\right)^{-n / 2}\right]^{-1 / n}
$$


In Equation (12.13a), the parameters are

$$
\begin{aligned}
& \mathrm{U}_{\mathrm{b}} \quad=\text { bubble rise velocity }(\mathrm{m} / \mathrm{s}) \\
& \mathrm{D}_{\mathrm{d}} \quad=\text { equivalent diameter }(\mathrm{m}) \text {; the diameter of a sphere having the same volume } \\
& \text { as the bubble } \\
& \sigma=\quad \text { liquid surface tension }(\mathrm{N} / \mathrm{m}) \\
& \text { g = acceleration of gravity }\left(\mathrm{m} / \mathrm{s}^{2}\right) \\
& \rho_{c} \quad=\text { density of liquid }\left(\mathrm{kg} / \mathrm{m}^{3}\right) \\
& \rho_{\mathrm{d}} \quad=\text { density of } \mathrm{gas}\left(\mathrm{kg} / \mathrm{m}^{3}\right) \\
& \mu_{\mathrm{c}} \quad=\text { viscosity of liquid }(\mathrm{Pa} \mathrm{s}) \\
& \mathrm{K}_{\mathrm{b}}=\mathrm{a} \text { constant equal to } \mathrm{K}_{\mathrm{b} 0} \mathrm{Mo}^{-0.038} \text {, where } \mathrm{K}_{\mathrm{b} 0} \text { is } 14.7 \text { for aqueous solutions or } \\
& M o=\frac{g\left(\rho_{c}-\rho_{d}\right) \mu_{c}^{4}}{\rho_{c}^{2} \sigma^{3}}
\end{aligned}
$$

Equation (12.13a) incorporates terms for the viscous, distorted, and spherical-cap regimes, interpolating between them with the exponent $n$ to cover all regimes. The range of applicable Morton numbers is from $10^{-12}$ to $10^{5}$. The submodel's demonstrated range of applicability extends from very small Eotvos numbers (corresponding to small spherical bubbles) to Eotvos numbers in excess of 40 (large spherical-cap bubbles). The Eotvos number Eo is defined in Equation (12.14).

$$
\mathrm{Eo}=\frac{g \rho_{c} D_{d}^{2}}{\sigma}
$$


The limitations of the Fan-Tsuchiya bubble rise submodel given in Equation (12.13) are 1) Newtonian fluids only; 2) single bubbles only; and 3) only liquids without any suspended particles. It is, however, applicable for most Newtonian fluids and bubble sizes in general engineering practice and has been confirmed against both theory and experiment.

The reliance of the Fan-Tsuchiya submodel on the experimental parameter $\mathbf{n}$ raises some doubt about its general applicability, but it is no less applicable in this regard than other models. The model proposed by Wallis (1974), for example, requires a similarly doubtful judgment of whether or not the fluid is free of surface-active solutes.

A single-bubble drag coefficient can be derived that is consistent with the Fan-Tsuchiya velocity model. When the bubble or particle reaches terminal velocity, the forces on it are balanced and the drag force equals the net buoyant force. The drag coefficient can therefore be defined as in Equation (12.15):

$$
\begin{aligned}
& C_{d}=\frac{\text { drag force } / \text { cross }- \text { sectional area }}{1 / 2 \rho_{c} U_{b}^{2}} \\
& C_{d}=\frac{\text { net buoyant force } / \text { cross }- \text { sec tional area }}{1 / 2 \rho_{c} U_{b}^{2}}=\frac{4\left(\rho_{c}-\rho_{d}\right) g D_{d}}{3 \rho_{c} U_{b}^{2}}
\end{aligned}
$$

Substituting Equation (12.13a) into Equation (12.15) yields Equation (12.16).

$$
C_{d}=\frac{4}{3} \frac{D_{d} g\left(\rho_{c}-\rho_{d}\right)}{\rho_{c}}\left[\left(\frac{D_{d}^{2} g\left(\rho_{c}-\rho_{d}\right)}{K_{b} \mu_{c}}\right)^{-n}+\left(\frac{2 c \sigma}{\rho_{c} D_{d}}+\frac{g D_{d}}{2}\right)^{-n / 2}\right]^{2 / n}
$$

Or, in a dimensionless form,

$$
C_{d}=\frac{4}{3} \frac{\left(\rho_{c}-\rho_{d}\right)}{\rho_{c}}\left(\frac{\rho_{c} D_{d}^{2} g}{\sigma}\right)^{1 / 2}\left[\left(\frac{E o}{K_{b} M o^{1 / 4}}\right)^{-n}+\left(\frac{2 c}{\sqrt{E o}}+\frac{\sqrt{E o}}{2}\right)^{-n / 2}\right]^{2 / n}
$$

It is not clear from the discussion given in Fan and Tsuchiya (1990) whether Equations (12.16a) and (12.16b) account for the density of the dispersed phase sufficiently to allow the 
equation to be used for dispersed liquid drops, as well as for gas bubbles. Because of this question, and because the correlating constants $n$ and $K_{b}$ are fluid-specific, such an extension is not advisable.

\subsubsection{Wallis submodel for single bubble rise}

The Wallis single-bubble rise submodel (Wallis 1974) explicitly defines the regimes within which certain types of bubble rise behavior occur. In the first regime, in which bubbles remain roughly spherical, the bubble rise velocity increases as diameter increases, as shown in Equation (12.17).

$$
\begin{array}{ll}
\mathrm{u}^{*}=0.222 \mathrm{r}^{* 2} & \text { for } \mathrm{r}^{*}<1.732 \\
\mathrm{u}^{*}=0.33 \mathrm{r}^{* 1.28} & \text { for } 1.732 \leq \mathrm{r}^{*}<5.803 \\
\mathrm{u}^{*}=0.693 \mathrm{r}^{* 0.858} & \text { for } 5.803 \leq \mathrm{r}^{*}<36 \\
\mathrm{u}^{*}=2.5 \sqrt{\mathrm{r}^{*}} & \text { for } \quad \mathrm{r}^{*} \geq 36
\end{array}
$$

The dimensionless rise velocity $u^{*}$ and bubble radius $r^{*}$ here are defined in Equations (12.18) and (12.19).

$$
\begin{aligned}
& U_{b}=u^{*}\left(\frac{\mu_{c} g\left(\rho_{c}-\rho_{d}\right)}{\rho_{c}^{2}}\right)^{1 / 3} \\
& r^{*}=\frac{1}{2} D_{d}\left(\frac{\rho_{c} g\left(\rho_{c}-\rho_{d}\right)}{\mu_{c}^{2}}\right)^{1 / 3} .
\end{aligned}
$$

Wallis (1974) made a distinction between bubbles with rigid interfaces and those with mobile interfaces, and provided two different algorithms for bubble rise. Equation (12.17) describes the spherical and near-spheriçal rigid bubbles, under the assumption that the high-purity conditions required for mobile or fluid bubbles are not present in tank wastes.

When a bubble becomes large enough, it may enter a regime (Wallis Region 4) in which the bubble is severely distorted and the rise velocity does not depend on the bubble diameter, as shown in Equation (12.20). 


$$
\mathrm{u}^{*}=\frac{1.56}{\mathrm{Mo}^{1 / 12}} \quad \text { for } \mathrm{u}^{*}>\frac{1.56}{\mathrm{Mo}^{1 / 12}}
$$

Finally, at the largest sizes, the bubble becomes a spherical cap (Wallis Region 5) and its rise velocity once again increases with diameter, as shown in Equation (12.21).

$$
u^{*}=\sqrt{r^{*}} \quad \text { for } u^{*}>\sqrt{\frac{6}{\mathrm{r}^{*} \mathrm{Mo}^{1 / 3}}}
$$

The most convenient way to use the Wallis method is to calculate the three possible rise velocities, using Equations (12.17), (12.20), and (12.21), and choose the proper value. Equation (12.22) gives the algorithm for this approach.

$$
\mathrm{u}^{*}=\text { minimum( Eqn. 12.17, maximum( Eqn. 12.20, 12.21 ) ) }
$$

The Fan-Tsuchiya submodel and the Wallis submodel are within about $30 \%$ of each other except in the spherical bubble regime, where a factor of two difference can be present. (Figure 12.1 shows sample bubble rise velocities for single bubbles rising through the solution in Tank SY-101, with properties of, roughly, $\rho_{c}=1500 \mathrm{~kg} / \mathrm{m}^{3}, \sigma=0.080 \mathrm{~N} / \mathrm{m}$, and $\mu_{c}=0.026 \mathrm{~Pa} \mathrm{~s}$.) The difference between the two models is less if the bubble-rise exponent for distilled water is used in the Fan and Tsuchiya submodel. This result suggests that the Wallis submodel [as described in Equations (12.17) through (12.22)] is based more strongly on the assumption of pure water and high bubble mobility than is the Fan-Tsuchiya submodel.

\subsubsection{Bubble swarm rise}

The factor to transform the rise velocity (or drag coefficient) for a single bubble to the rise velocity for a swarm of bubbles is a function of the gas-phase volume fraction $\varepsilon_{\mathrm{d}}$. Ishii and Chawla (1979) derived relations for rise velocities and drag coefficients of bubble swarms in the full range of regimes, and provided experimental data that showed good agreement. Because these relations are specified for each bubbly-flow regime, it is possible to incorporate them directly into Equation (12.16), or into Equations (12.17) through (12.21), if the Wallis submodel is preferred. 


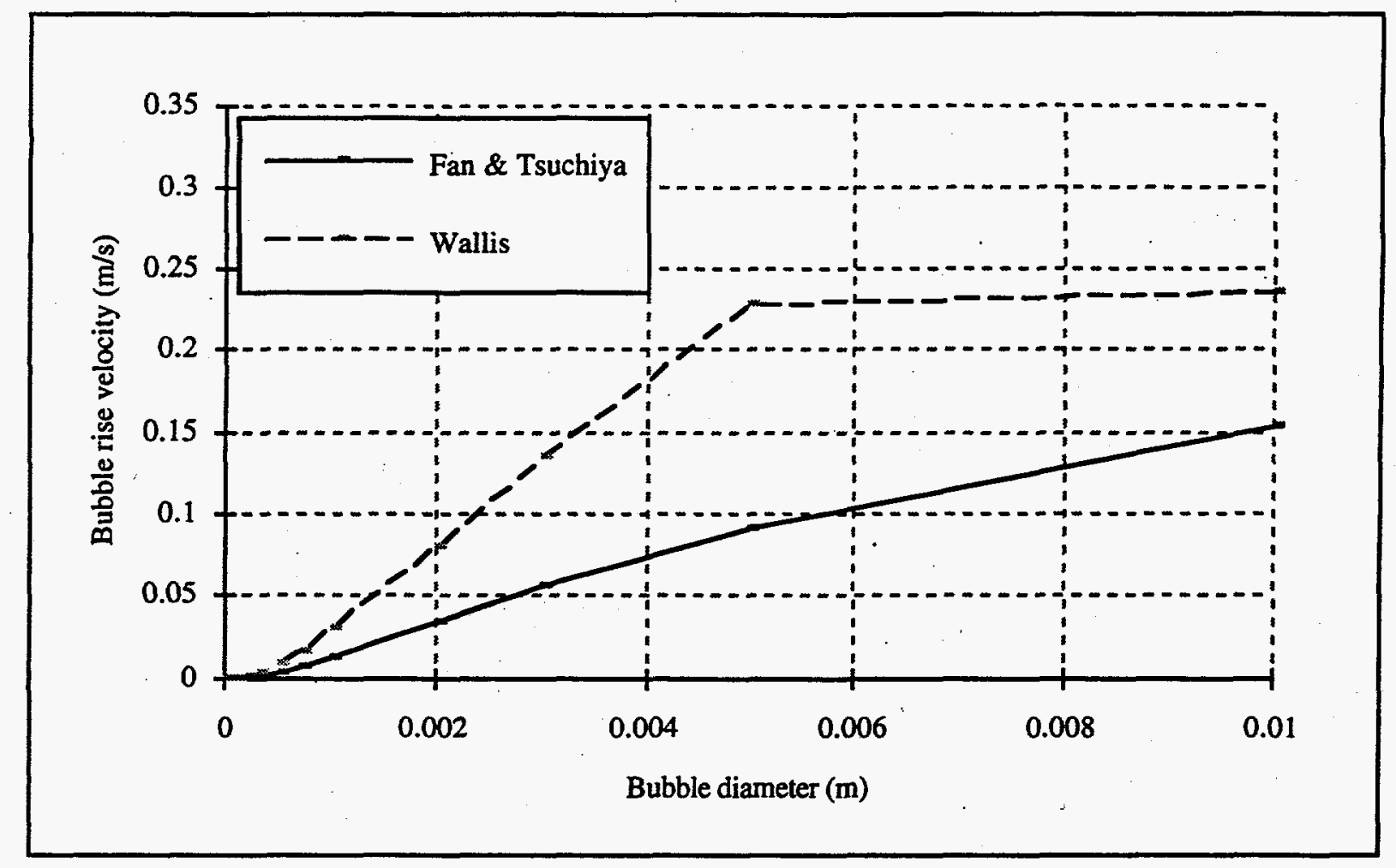

Figure 12.1. Comparison of single-bubble rise submodels.

In the viscous regime, for the smallest bubble sizes, Ishii and Chawla demonstrate the drag coefficient can be derived by substituting a mixture viscosity $\mu_{m}$ for the pure-fluid viscosity $\mu_{c}$. For bubbly flow, the mixture viscosity can be expressed as in Equation (12.23).

$$
\begin{aligned}
& \frac{\mu_{\mathrm{m}}}{\mu_{\mathrm{c}}}=\left(1-\frac{\varepsilon_{\mathrm{d}}}{\varepsilon_{\mathrm{d}, \mathrm{m}}}\right)^{-\varepsilon_{\mathrm{d}, \mathrm{m}}} \\
& \text { where } \varepsilon_{\mathrm{d}} \quad=\text { gas-phase volume fraction } \\
& \varepsilon_{\mathrm{d}, \mathrm{m}} \quad=\text { maximum-packing gas volume fraction, taken as } 0.95 .
\end{aligned}
$$

The high maximum-packing fraction for bubbles allows the consideration of flow in foams, though no systems of this kind were tested. When this mixture viscosity is substituted into the first term of the bracketed expression in Equation (12.16a), the term is modified to the form given in Equation (12.24). 


$$
\left(\frac{D_{d}^{2} g\left(\rho_{c}-\rho_{d}\right)}{K_{b} \mu_{m}}\right)^{-n}
$$

If the Wallis (1974) submodel is used, the mixture viscosity $\mu_{m}$ can be substituted into. Equations (12.17), (12.18), and (12.19), which together describe creeping and near-creeping bubble rise.

The second term in the Fan-Tsuchiya submodel describes distorted bubbles, for which the large eddies in the wake contribute most of the effect on the drag coefficient. The second term is multiplied by a function of the void fraction (Equation 38 of Ishii and Chawla). This function is chosen to provide consistent values at the transition between the distorted-particle regime and the viscous regime. The new second term in the Fan and Tsuchiya submodel is given in Equation (12.25).

$$
\left(\frac{2 c \sigma}{\rho_{c} D_{d}}\left(\frac{18.67\left(\mu_{c} / \mu_{m}\right) \sqrt{1-\varepsilon_{d}}}{1+17.67\left[\left(\mu_{c} / \mu_{m}\right) \sqrt{1-\varepsilon_{d}}\right]^{6 / 7}}\right)^{2}+\ldots\right)^{-\mathrm{n} / 2}
$$

The predicted rise velocities for a variety of gas-liquid systems in the distorted regime were compared to data over a wide range of void fractions, and the equation predicted the bubbles' rise closely.

Finally the third term of the Fan-Tsuchiya submodel, the term for spherical-cap bubbles, is modified per Equation 47 of Ishii and Chawla (1979) to become that shown in Equation (12.26).

$$
\left(\ldots+\frac{g D_{d}}{2\left(1-\varepsilon_{d}\right)^{2}}\right)^{-n / 2}
$$


The full equation for the drag coefficient in a bubbly fluid, as described by the ensemble version of the Fan and Tsuchiya submodel, is then given by Equation (12.27).

$$
C_{d}=\frac{4}{3} \frac{D_{d} g\left(\rho_{c}-\rho_{d}\right)}{\rho_{c}}\left[\left(\frac{D_{d}^{2} g\left(\rho_{c}-\rho_{d}\right)}{K_{b} \mu_{m}}\right)^{-n}+\left(\frac{2 c \sigma}{\rho_{c} D_{d}}\left(\frac{18.67\left(\mu_{c} / \mu_{m}\right) \sqrt{1-\varepsilon_{d}}}{1+17.67\left[\left(\mu_{c} / \mu_{m}\right) \sqrt{1-\varepsilon_{d}}\right]^{6 / 7}}\right)^{2}+\frac{g D_{d}}{2\left(1-\varepsilon_{d}\right)^{2}}\right)^{-n / 2}\right]^{2 / n}
$$

where all the quantities have already been defined. The dimensionless form of this equation is

$$
C_{d}=\frac{4}{3} \frac{\left(\rho_{c}-\rho_{d}\right)}{\rho_{c}}\left(\frac{\rho_{c} D_{d}^{2} g}{\sigma}\right)^{1 / 2}\left[\left(\frac{E o}{K_{b} M o_{m}^{1 / 4}}\right)^{-n}+\left(\frac{2 c}{\sqrt{E o}}\left(\frac{18.67\left(\mu_{c} / \mu_{m}\right) \sqrt{1-\varepsilon_{d}}}{1+17.67\left[\left(\mu_{c} / \mu_{m}\right) \sqrt{1-\varepsilon_{d}}\right]^{6 / 7}}\right)^{2}+\frac{\sqrt{E o}}{2\left(1-\varepsilon_{d}\right)^{2}}\right)^{-n / 2}\right]^{2 / n}
$$

Here the mixture Morton number $\left(\mathrm{Mo}_{\mathrm{m}}\right)$ is calculated with the mixture viscosity from Equation (12.17), instead of the pure fluid viscosity $\mu_{c}$.

It is questionable whether the exponent $n$ that was defined for the single-bubble case is the best regime-interpolating parameter at higher void fractions. However, an advantage of using the same value of $\mathrm{n}$ is that Equation (12.21) reduces to Equation (12.16) as the void fraction approaches zero. No more detailed information is available to modify the value of $n$.

For the Wallis submodel, the application of the Ishii and Chawla (1979) ensemble corrections is less evident. Probably the most appropriate approach is to multiply Equations (12.20) and (12.21) by $1 /\left(1-\varepsilon_{d}\right)$, treating both as spherical cap regime. This avoids a discontinuity of the rise velocity between the two equations. Figure 12.2 shows the behavior of the Fan-Tsuchiya and the Wallis submodels, as modified to include ensemble (or swarm) effects. The differences between the two submodels appear to be increased by the ensemble modifications. 


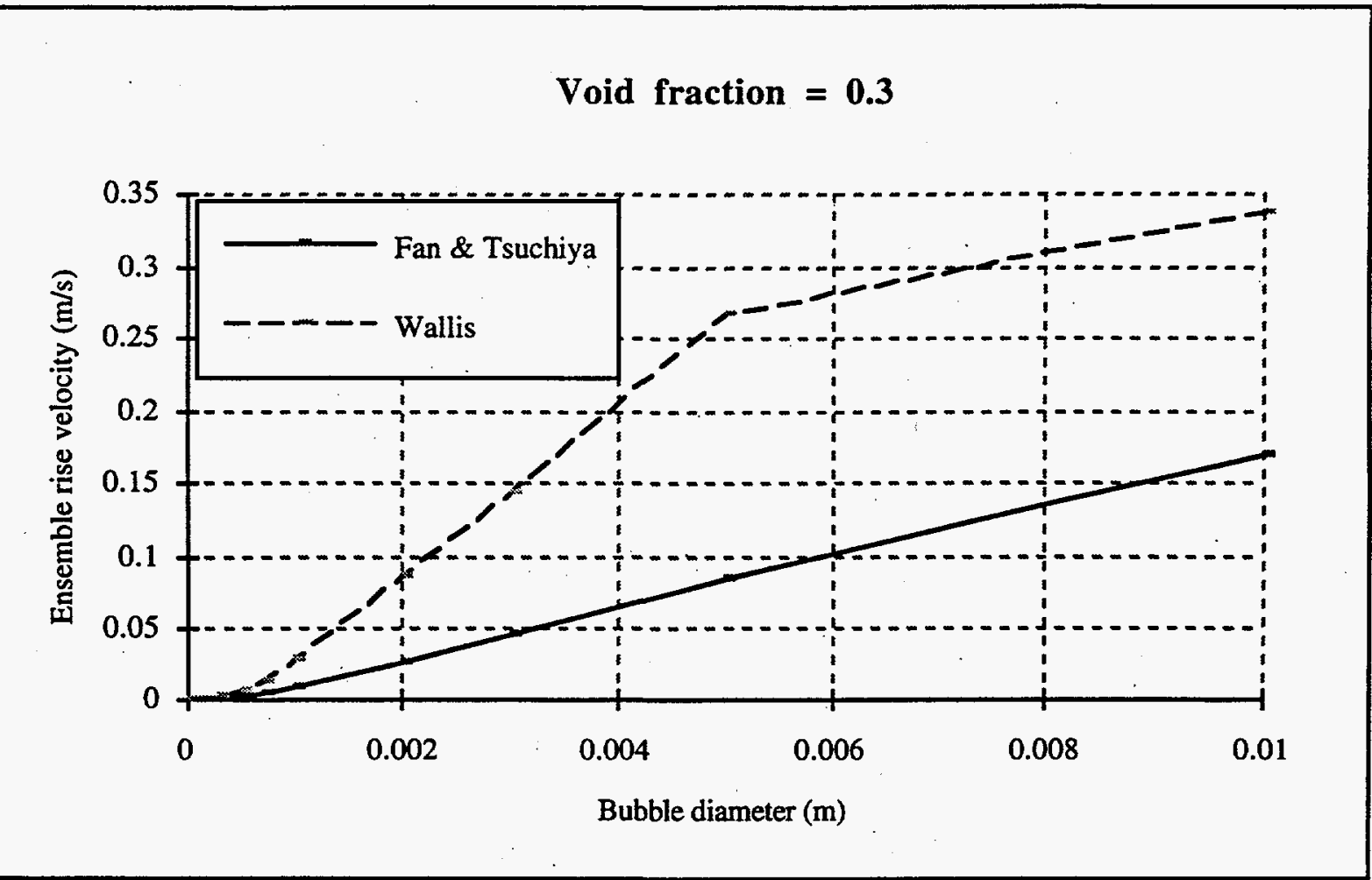

Figure 12.2. Comparison of bubble swarm rise submodels.

\subsubsection{Rise in non-homogeneous or non-Newtonian fluids}

Two gaps remaining in the bubble drag calculation method are the effect of the particles in the sludge or supernatant on the bubble rise, and the possibility the particle-free supernatant itself is non-Newtonian.

Ishii and Chawla (1979) described particle drag coefficients as having very similar relationships to bubble drag coefficients, and derived very similar formulas for the mixture viscosities of fluids containing bubbles and particles. Equation (12.28) gives their mixture viscosity for particles.

$$
\frac{\mu_{m}}{\mu_{c}}=\left(1-\frac{\varepsilon_{s}}{\varepsilon_{s, m}}\right)^{-2.5 \varepsilon_{s, m}}
$$




$$
\begin{aligned}
& \text { where } \begin{array}{ll}
\mu_{\mathrm{m}} & =\text { mixture viscosity } \\
\mu_{\mathrm{c}} & =\text { pure fluid viscosity } \\
\varepsilon_{\mathrm{s}} & =\text { volume fraction of particles } \\
\varepsilon_{\mathrm{s}, \mathrm{m}} \quad= & \text { maximum-packing solid volume fraction, typically between } 0.5 \text { and } 0.74,
\end{array}
\end{aligned}
$$

Ishii and Chawla (1979) also supplied drag coefficient relationships for particles (their Equations 34 and 36). These two formulas, for the viscous and Newtonian regimes respectively, can be combined in the same way that Fan and Tsuchiya (1990) combined the separate terms for bubble rise. The result is as shown in Equation (12.29).

$$
C_{d}=\left(\left(\frac{24\left(1+0.1 \operatorname{Re}_{m}^{0.75}\right)}{\operatorname{Re}_{m}}\right)^{n}+\left(0.45\left(\frac{1+17.67\left[\left(\mu_{c} / \mu_{m}\right) \sqrt{1-\varepsilon_{s}}\right]^{6 / 7}}{18.67\left(\mu_{c} / \mu_{m}\right) \sqrt{1-\varepsilon_{s}}}\right)^{2}\right)^{n}\right)^{1 / n}
$$

where $\operatorname{Re}_{\mathrm{m}}=$ the particle Reynolds number calculated using the mixture viscosity, that is,

$$
\operatorname{Re}_{\mathrm{m}}=\mathrm{V}_{\mathrm{s}} \mathrm{D}_{\mathrm{s}} \rho_{\mathrm{c}} / \mu_{\mathrm{m}}
$$

$$
\begin{aligned}
& V_{s} \quad=\text { particle terminal velocity } \\
& D_{s} \quad=\text { particle effective diameter } \\
& \rho_{c} \quad=\text { pure fluid density } \\
& n \quad \quad=7.5 \text { for an adequately smooth interpolation between regimes }
\end{aligned}
$$

Because the particle terminal velocity is calculated from the drag coefficient, iteration of the velocity and Reynolds number is required to arrive at a final value of $C_{d}$. If the first value guessed for $C_{d}$ is the Stokes value ( $\left.24 / R_{m}\right)$, two iterations suffice to find a value of $C_{d}$ that gives a settling velocity within $10 \%$ of the fully converged value. 
One possible approach to calculating the bubble and particle drag coefficients in the presence of both bubbles and particles would be to follow the line of reasoning proposed in Gauglitz et al. (1994a). Then the bubbly slurry viscosity would be as shown in Equation (12.30):

$$
\frac{\mu_{m}}{\mu_{c}}=\left(1-\frac{\varepsilon_{s}}{\varepsilon_{s, m}}\right)^{-2.5 \varepsilon_{s, m}}\left(1-\frac{\varepsilon_{d}}{1-\varepsilon_{s}}\right)^{-2.5}
$$

This approach is opportune, but imperfect, because it accounts for volume exclusion, but not for the increased drag caused by the opposite directions of bubble and particle motion.

Another mixture-viscosity approach was detailed by Fan and Tsuchiya (1990, p. 49). A water-fluidized bed or suspension of particles can be treated as a homogeneous medium (a liquid of higher density and viscosity than water) under certain conditions, for low solids content (less than about 50 vol\%) and small particle size $D_{s}$, compared to bubble size $D_{d}\left(D_{d} / D_{s}>30\right)$. Both the solids fraction and particle size criteria appear to be met by the tank supernatant. The reported solid fractions for the top and middle layers of SY-101 (18 vol\% and $28 \mathrm{vol} \%)$ meet the first criterion, though the 60 vol\% solids content at the bottom does not. The particles in the SY-101 waste have been reported to have a log-normal mode of about $4 \mu \mathrm{m}$, with a few particles as large as $300 \mu \mathrm{m}$ (Chang and Beaver 1993). The bubble size is unknown, although the bubbles that have been seen at the top of the tank are definitely large compared to the sludge particles.

Fan and Tsuchiya (1990) give experimentally derived correlations to estimate the increased density and viscosity of the homogeneous suspension:

$$
\begin{aligned}
& \rho_{m}=\rho_{c}\left(1-\varepsilon_{s}\right)+\rho_{s} \varepsilon_{s} \\
& \mu_{m}=\mu_{c} \exp \left(36.15 \varepsilon_{s}^{2.5}\right) \quad \text { for } \varepsilon_{s}>0.2
\end{aligned}
$$

$$
\begin{aligned}
& \text { where } \varepsilon_{\mathrm{s}} \quad=\text { solid volume fraction in suspension } \\
& \rho_{\mathrm{s}} \quad \begin{array}{l}
\text { particle density (which should be less than } 3 \mathrm{~g} / \mathrm{cc} \text { for this correlation; this } \\
\text { limit is in accord with the density of the solids in tank SY-101) }
\end{array}
\end{aligned}
$$

The values for $\rho_{m}$ and $\mu_{m}$ that are given by Equations (12.31) and (12.32) could be used instead of $\rho_{c}$ and $\mu_{c}$ in Equations (12.13) through (12.29). This simple treatment of a suspension as a homogeneous fluid has its limitations; however, under SY-101 conditions, the potential error in the 
rise velocity is probably less than $50 \%$. The improvement in predictive ability that might be attained with a much more complicated approach, such as that discussed in Jang (1989), is not great enough to warrant the effort.

A third possible approach to estimating bubble and particle rise velocities in bubbly suspensions can be derived from an equation given by Perry and Chilton (1973) for the effect of solids concentration on the settling velocity of a particle:

$$
\begin{aligned}
& \mathrm{V}_{\mathrm{s}, \mathrm{h}}=\mathrm{V}_{\mathrm{s}}\left(1-\mathrm{C}_{\mathrm{r}}\right) \mathrm{m} \\
& \text { where } \begin{aligned}
\mathrm{V}_{\mathrm{s}, \mathrm{h}}= & \text { hindered settling velocity of a particle in a suspension }(\mathrm{m} / \mathrm{s}) \\
\mathrm{V}_{\mathrm{s}}= & \text { settling velocity of an isolated particle }(\mathrm{m} / \mathrm{s}) \\
\mathrm{C}_{\mathrm{r}}= & \text { reduced concentration, or the volume fraction solids divided by the } \\
& \text { volume fraction at maximum packing; equal to } \varepsilon_{\mathrm{s}} / \varepsilon_{\mathrm{s}, \mathrm{m} \cdot} \\
\mathrm{m} & \text { an exponent that equals } 4.65 \text { for the Reynolds number } \operatorname{Re}<0.3 \text {, and } \\
& 2.33 \text { for } \operatorname{Re}>1000 \text {. In the intermediate region, a log-log interpolation } \\
& \text { can be used to find } \mathrm{m} \text { (Perry and Chilton, Equation } 5-224, \text { Figure } 5-83) . \\
= & V_{s} D_{s} \rho d \mu
\end{aligned}
\end{aligned}
$$

Equation (12.26) could be extended to describe bubbles and particles in a bubbly suspension. The form of the extended submodel would be

$$
\mathrm{V}_{\mathrm{s}, \mathrm{b}}=\mathrm{V}\left(1-\frac{\varepsilon_{\mathrm{s}}}{\varepsilon_{\mathrm{s}, \mathrm{m}}}\right)^{\mathrm{m}}\left(1-\frac{\varepsilon_{\mathrm{d}}}{1-\varepsilon_{\mathrm{s}}}\right)^{\mathrm{m}}
$$

where $V_{s, b}=$ the hindered settling velocity of a particle, or the hindered rise velocity of a bubble, in a bubbly suspension

$\mathrm{V}=$ either the settling velocity $\mathrm{V}_{\mathrm{s}}$ for a particle, or the rise velocity $U_{b}$ for $a$ bubble

$\varepsilon_{\mathrm{s}} \quad=$ solids volume fraction

$\varepsilon_{\mathrm{s}, \mathrm{m}}=$ maximum packed solids volume fraction

$\varepsilon_{\mathrm{d}} \quad=$ gas volume fraction, or void fraction

Though the submodel in Equation (12.27) is plausible, no data were found to support using the same value of $\mathrm{m}$ for a bubbly suspension as for a gasless suspension. 
With all three of these submodels, the major uncertainties would be a) the particle sizes are smaller and solid fractions higher than those used in the originating experiments, and $b$ ) the fluid is not water but a more viscous liquid. In addition, these submodels shed no light on the effect of bubbles on the particle settling velocity and do not prescribe a method for estimating the effect of the presence of bubbles on particle velocities.

On the whole, the model for bubbly slurry bulk viscosity that was proposed by Gauglitz et al. (1994a) is probably the best of the available options. Equation (12.30) is recommended for use in all of the equations in this chapter where a bulk viscosity is needed, whether for bubble swarms, particles, or mixtures of the two. Note that Equation (12.30) is being treated as applicable for the purpose of estimating bubble rise velocities; other forms may be preferable for calculating viscosity for use in modeling macroscopic flows. For the latter subject, see Section 9.0.

The supernatant fluid may be non-Newtonian. If s.o, it is probably a shear-thinning (pseudoplastic) fluid that can be represented by a power-law relation. In general, bubble velocity increases more rapidly with bubble size for pseudoplastic fluids than for Newtonian fluids. However, the effect of void fraction in a swarm is less for pseudoplastic fluids.

Some theoretical work has been done by Manjunath and Chhabra (1992) on the rise of bubble swarms in power-law fluids. However, their solution was purely numerical and did not provide a correlation that could be incorporated into an analytical tool. An earlier paper by Bhavaraju et al. (1978a, b) gave some extremely complicated theoretical expressions that could be used to calculate a drag coefficient for a swarm of non-rigid bubbles in creeping flow in a power-law fluid that was slightly non-Newtonian. The error of these predictions was less than $20 \%$, as long as the exponent $n$ was between 0.7 and 1 in the power-law expression. It is not clear that these expressions would be sufficiently applicable to be worth the effort, because the bubbles in the tank are likely to be outside the creeping flow range and are very unlikely to have clean interfaces.

\subsection{Virtual mass acceleration}

The virtual mass force is, roughly speaking, the force the bubble must exert to accelerate the volume of liquid above it out of its way. It is dependent on bubble volume, fluid density, and the local void fraction.

The virtual mass force per unit bubble volume $\mathbf{F}_{\mathrm{vm}}$ is expressed as shown in Equation (12.26). 


$$
\mathbf{F}_{\mathrm{vm}}=\mathrm{C}_{\mathrm{vm}} \rho_{\mathrm{c}} \mathbf{a}_{\mathrm{R}}
$$

where $\mathrm{C}_{\mathrm{vm}}=$ the virtual mass coefficient

$\rho_{c} \quad=$ the liquid density

$\mathbf{a}_{\mathrm{R}} \quad=$ the relative mass acceleration of the bubble in the fluid

For a single non-deformable spherical bubble at a void fraction of 0 , kinetic energy considerations lead to a $C_{v m}$ value of $1 / 2$ [Drew et al. (1979)]. The values at void fractions greater than zero have been investigated in a few studies. Under experimental conditions where bubbles were spheres or slightly oblate spheroids, Ruggles (1987) found the dependence stated in Equation (12.27).

$$
C_{v m}=C_{v m 0}\left(1+12 \varepsilon_{d}^{2}\right) \quad \text { for } \varepsilon_{d} \leq 0.2
$$

In Equation (12.27), $\mathrm{C}_{\mathrm{vm} 0}$ is the virtual mass coefficient at zero void fraction. No information seems to be available on the void fraction variation for $\varepsilon_{\mathrm{d}}$ greater than $20 \%$.

In addition, $\mathrm{C}_{\mathrm{vmo}}$ is dependent on the shape of the bubble. Figure 2.5 of Fan and Tsuchiya (1990) shows that bubbles in fluid as viscous as the tank supernatant change in shape from a sphere to an oblate ellipsoid (with a minimum aspect ratio of 0.2 ) to a spherical cap, as the bubble volume increases. A value for $C_{v m 0}$ can be derived based on the theory given in Lamb (1932) (articles 113, 114, and 700). Lamb (1932) gives an expression for the virtual mass coefficient of an oblate ellipsoid moving parallel to its short axis. This expression is quoted in Equation (12.28):

$$
C_{\mathrm{vm} 0}=\frac{\left(\zeta^{2}+1\right)\left(1-\zeta \cot ^{-1} \zeta\right)}{1-\left(\zeta^{2}+1\right)\left(1-\zeta \cot ^{-1} \zeta\right)}
$$

In Equation (12.28), $\zeta$ is the ellipsoidal coordinate, and is defined by Equation (12.29).

$$
\zeta=\sqrt{\frac{1}{\mathrm{~b}^{2} / \mathrm{h}^{2}-1}}
$$




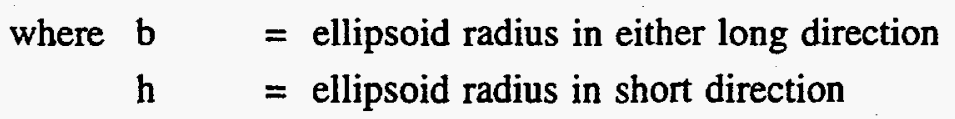

The application of equation (12.28) to an oblate ellipsoidal bubble is evident. It can be adapted to a spherical cap bubble of radius $b$ and height $h$ by assuming that a spherical cap has the same $C_{v m 0}$ as an oblate ellipsoid with the same $b$ and $h$. For the spherical cap, the ratio $b / h$ depends on the included angle of the cap. For the usual spherical cap with an included angle of 100 degrees [Fan and Tsuchiya ) 1990)], the aspect ratio $\mathrm{h} / \mathrm{b}$ is $0.47, \zeta=0.53$, and $\mathrm{C}_{\mathrm{vm} 0}=1.2$. Data in Section 2.1C and Figure 2.6(b) of Fan and Tsuchiya (1990) support the correlation given in Equation (12.30).

$$
\begin{aligned}
& \frac{b}{h}=\frac{0.175}{1+E 0^{4}}+0.34+6.91 \times 10^{-4}(E o-26.5)^{2} \quad \text { for } E o<40 \\
& \frac{b}{h}=0.466 \quad \text { for } E o \geq 40
\end{aligned}
$$

The most widely accepted correlations for bubble shape are expressed in terms of the bubble velocity, rather than the Eotvos number, but these correlations would require some iteration to solve for both bubble velocity and shape. Therefore, a correlation using Eotvos number seems more convenient. The correlation in Equation (12.30) matches data for air bubbles in contaminated liquids with low Morton numbers (less than about 10-3).

Thus, a value for $\mathrm{C}_{\mathrm{vm}}$ can be obtained by using the Eotvos number (Equation 12.14) to estimate $b / h$ (Equation 12.30), from which $\zeta$ can be calculated (Equation 12.29), which permits calculation of $\mathrm{C}_{\mathrm{vm} 0}$ (Equation 12.28). Then $\mathrm{C}_{\mathrm{vm}}$ can be estimated from $\mathrm{C}_{\mathrm{vm} 0}$ and $\varepsilon_{\mathrm{d}}$ using Equation (12.27).

Drew et al. (1979) also derived the most general objective (frame-invariant) expression for the virtual mass acceleration $\mathbf{a}_{\mathbf{R}}$ :

$$
\mathbf{a}_{\mathrm{R}}=\frac{\mathrm{D}_{\mathrm{d}}}{\mathrm{Dt}}\left(\mathbf{u}_{\mathrm{d}}-\mathbf{u}_{\mathrm{c}}\right)+\left(\mathbf{u}_{\mathrm{d}}-\mathbf{u}_{\mathrm{c}}\right) \cdot\left[(\lambda-2) \nabla \mathbf{u}_{\mathrm{d}}+(1-\lambda) \nabla \mathbf{u}_{\mathrm{c}}\right]
$$


In Equation (12.31), the total derivative operator for the dispersed phase (gas) is defined by

$$
\frac{\mathrm{D}_{\mathrm{d}}}{\mathrm{Dt}}=\frac{\partial}{\partial \mathrm{t}}+\mathbf{u}_{\mathrm{d}} \cdot \boldsymbol{\nabla}
$$

The parameter $\lambda$ in Equation (12.32) depends on the volume fraction of gas.

A simpler objective formulation has also been devised(18):

$$
a_{R}=\frac{\partial}{\partial t}\left(\mathbf{u}_{d}-\mathbf{u}_{c}\right)+\nabla \cdot \mathbf{u}_{d}\left(\mathbf{u}_{d}-\mathbf{u}_{c}\right)
$$

These two formulations of virtual acceleration are equal for $\lambda=1$. Because the parameter $\lambda\left(\varepsilon_{\mathrm{d}}\right)$ varies from 2 at very low void fraction to 0 at very high void fraction, and its exact void fraction variation is unknown, it seems reasonable to use the less complicated equation (12.33).

(18) Stewart, C.W. 1993. "NFIELD: A General Computational Model for Simulation of Multi-Field, Multi-Component, Multi-Dimensional Fluid Flows." Personal communication. 


\subsection{References and Resources}

\subsection{References}

Berker, A., and W.E. Van Arsdale. 1992. "Phenomenological models of viscoplastic, thixotropic, and granular materials." Rheol. Acta 31:119-138.

Bhavaraju, S.M., R.A. Mashelkar, and H.W. Blanch. 1978a. "Bubble Motion and Mass Transfer in Non-Newtonian Fluids. Part 1. Single Bubble in Power Law Fluid." AIChE J. 24(6):1070-1076.

Bhavaraju, S.M., R.A. Mashelkar, and H.W. Blanch. 1978b. "Bubble Motion and Mass Transfer in Non-Newtonian Fluids. Part 2. Swarm of Bubbles in a Power Law and Bingham Fluids." AIChE J. 24(6):1063-1070.

Bird, R.B., G.C. Dai, and B.J. Yarusso. 1983. "The Rheology and Flow of Viscoplastic Materials." Rev. Chem. Eng. 1(1):1-70.

Bird, R.B., W.E. Stewart, and E.N. Lightfoot. 1960. Transport Phenomena. John Wiley \& Sons, Inc., New York, New York.

Bredt, P.R., E.J. Eschbach, J.D. Hudson, H.C. Reid, and K.R. Recknagle. 1994. Technical Summary of Data Related to Dilution of Tank 241-SY-101 Wastes. PNL-MIT52394, Pacific Northwest Laboratory, Richland, Washington. May 23, 1994.

Ceo, R.N., M.B. Sears, and J.T. Shor. 1990. Physical Characterization of Radioactive Sludges in Selected Melton Valley and Evaporator Facility Storage Tanks. ORNL/TM-11653, Oak Ridge National Laboratory, Oak Ridge, Tennessee.

Chang, C., and R.L. Powell. 1994. "Effect of particle size distributions on the rheology of concentrated bimodal suspensions." J. Rheol. 38(1):85-98.

Cross, M.M. 1965. "Rheology of Non-Newtonian Fluids: A New Flow Equation for Pseudoplastic Systems." J. Colloid Sci. 20:417-437.

CRC. 1975. CRC Handbook of Chemistry and Physics, 56th Edition. CRC Press, Inc., Cleveland, Ohio. 
Dabak, T., and O. Yucel. 1987. "Modeling of the Concentration and Particle Size Distribution Effects on the Rheology of Highly Concentrated Suspensions." Powder Technol. 52:193-206.

Dean, J.A. 1985. Lange's Handbook of Chemistry, McGraw-Hill, New York, New York.

Drew, D., L. Cheng, and R.T. Lahey, Jr. 1979. "The Analysis of Virtual Mass Effects in Two-Phase Flow." Int. J. Multiphase Flow 5:233-242.

Ellwood, K.R.J., G.C. Georgiou, T.C. Papanastasiou, and J.O. Wilkes. 1990. "Laminar jets of Bingham-plastic liquids." J. Rheol. 34(6):787-813.

Evans, I.D. 1992. "Letter to the editor: On the nature of the yield stress." J. Rheol. 36(7):13131316.

Eyler, L.L., D.S. Trent, and J.A. Fort. 1993. TEMPEST: A Computer Program for ThreeDimensional Time-Dependent Computational Fluid Dynamics. Volume 2: User's Manual. Version T.Mod 2. PNL-8857,.Rev. 1, Pacific Northwest Laboratories, Richland, Washington.

Faddick, R.R. 1974. "Properties of Coal-Water Slurries." NTIS PB-251 721, Colorado School of Mines, Golden, Colorado. Prepared for Bureau of Mines.

Fam, D., J.A. Dodds, D. Leclerc, and O. Scrivener. 1989. "Rheological characterization and pipe flow of phosphate muds." Int. Chem. Engr. 29(3):455-463.

Fan, L.-S., and K. Tsuchiya. 1990. Bubble Wake Dynamics in Liquids and Liquid-Solid Suspensions. Butterworth-Heinemann, Boston, Massachusetts. pp. 39-52.

Felmy, A.R. 1990. GMIN: A Computerized Chemical Equilibrium Model Using a Constrained Minimization of the Gibbs Free Energy. PNL-7281, Pacific Northwest Laboratory, Richland Washington. April 1990.

Fow, C.L., D. McCarthy, and G.T. Thornton. 1986. "Rheological Evaluation of Simulated Neutralized Current Acid Waste." PNL-5820, Pacific Northwest Laboratory, Richland, Washington.

Gauglitz, P.A., R.R. Shah, and R.L. Davis. 1994a. Gas Distribution Effects on Waste Properties: Viscosities of Bubbly Slurries. PNL-10112, Pacific Northwest Laboratory, Richland, Washington. 
Gauglitz, P.A., L.A. Mahoney, D.P. Mendoza, and M.C. Miller. 1994b. Mechanisms of Gas Bubble Retention. PNL-10120, Pacific Northwest Laboratory, Richland, Washington.

Hadley, G.R. 1986. "Thermal conductivity of packed metal powders." Int. J. Heat Mass Transfer 29(6):909-920.

Himmelblau, D.M. 1974. Basic Principles and Calculation in Chemical Engineering, 3rd Edition. Prentice-Hall, Inc., Englewood Cliffs, New Jersey.

Husband, D.M., N. Aksel, and W. Gleissle. 1993. "The existence of static yield stresses in suspensions containing noncolloidal particles." J. Rheol. 37(2):215-235.

Ishii, M., and T.C. Chawla. 1979. Local Drag Laws in Dispersed Two-Phase Flow, NUREG/CR-1230, ANL-79-105, Argonne National Laboratory, Argonne, Illinois.

Jang, C.-S. 1989. Hydrodynamics of Liquid-Solid Fluidization. M.S. Thesis, Ohio State Univ., Columbus, Ohio.

Janz, G.J., B.G. Oliver, G.R. Lakshminarayanan, and G.E. Mayer. 1970. "Electrical Conductance, Diffusion, Viscosity, and Density of Sodium Nitrate, Sodium Perchlorate, and Sodium Thiocyanate in Concentrated Aqueous Solutions." J. Phys. Chem. 74(6):1285-1289.

Kalyon, D.M., P. Yaras, B. Aral, and U. Yilmazer. 1993. "Rheological behavior of a concentrated suspension: A solid rocket fuel simulant." J. Rheol. 37(1):35-53.

Kytomaa, H.K. 1992. "Liquefaction and Solidification." Particulate Two-Phase Flow Ed. by M. Roco, Butterworth-Heinemann, Boston Massachusetts.

Lamb, H. 1932. Hydrodynamics. Dover Publications, New York, New York.

Leavell, D.A., and J.F. Peters. 1987. Uniaxial Tensile Test for Soil. Technical Report GL-87-10, Department of the Army, Waterways Experimental Station, Vicksburg, Mississippi.

Manjunath, M., and R.P. Chhabra. 1992. "Free Rise Velocity of a Swarm of Spherical Gas Bubbles Through a Quiescent Power Law Liquid." Int. J. Eng. Sci. 30(7):871-878. 
Mewis, J. 1979. "Thixotropy -- A General Review." J. Non-Newtonian Fluid Mech. 6:1-20.

Nakamura, M. 1982. "A method to improve the effective-medium theory towards percolation problem." J. Phys. C 15:L749-L752.

Nakamura, M. 1984. "Conductivity for the site-percolation problem by an improved effectivemedium theory." Phys. Rev. B 29(6):3691-3693.

Nan, C.-W., and D.M. Smith. 1991. “A.C. electrical properties of composite solid electrolytes." Mater. Sci. Eng. B 10:99-106.

Nguyen, Q.D, and D.V. Boger. 1992. "Measuring the Flow Properties of Yield Stress Fluids." Annu. Rev. Fluid Mech. 24:47-88.

Nimick, F.B., and J.R. Leith. 1992. "A Model for Thermal Conductivity of Granular Porous Media." J. Heat Trans. 114:505-508.

Norton, J.D., and L.R. Pederson. 1994. Ammonia in Simulated Hanford Double-Shell Tank Wastes: Solubility and Effects on Surface Tension. PNL-10173, Pacific Northwest Laboratory, Richland, Washington.

Nozad, I., R.G. Carbonell, and S. Whitaker. 1985a. "Heat Conduction in Multiphase Systems -I. Theory and Experiment for Two-Phase Systems." Chem. Eng. Sci. 40(5):843-855.

Nozad, I., R.G. Carbonell, and S. Whitaker. 1985b. "Heat Conduction in Multiphase Systems -II. Experimental Method and Results for Three-Phase Systems." Chem. Eng. Sci. 40(5):857-863.

Ochs, T., C.G. Stojanoff, D.L. Day, E. Eckert, J. Langeliers, and D. Wruck. 1981. “Analysis of Saturated Solar Pond Characteristics." DOE/CS/30174--T2, Vol. 1, Desert Research Institute, Boulder City, Nevada.

Okuzono, T., K. Kawasaki, and T. Nagai. 1993. "Rheology of random foams." J. Rheol. 37(4):571-586.

Pasamehmetoglu, K.O. 1995. "Gas release estimates during retrieval operations in Tank 241-SY-101." TSA6-CN-WT-SA-GR-036/Rev. 1, Los Alamos National Laboratory, Los Alamos, New Mexico. February 1995. 
Perry, R.H., and C.H. Chilton. 1973. Chemical Engineers' Handbook. 5th Ed. McGraw-Hill Book Company, New York, New York.

Pietsch, W., E. Hoffman, and H. Rumpf. 1969. "Tensile Strength of Moist Agglomerates." Ind. Eng. Chem. Prod. Res. Dev. 8(1):58-62.

Probstein, R.F., M.Z. Sengun, and T.-C. Tseng. 1994. "Bimodal model of concentrated suspension viscosity for distributed particle sizes." J. Rheol. 38(4):811-829.

Reynolds, D.A., and D.L. Herting. 1984. Solubilities of Sodium Nitrate, Sodium Nitrite, and Sodium Aluminate in Simulated Nuclear Waste. RHO-RE-ST-14 P, Rockwell Hanford Operations, Richland, Washington. September 1984.

Ruggles, A.E. 1987. The Propagation of Pressure Perturbations in Bubble Air/Water Flows. Ph.D. thesis, Rensselaer Polytechnic Institute, Troy, New York.

Schubert, H. 1975. "Tensile Strength of Agglomerates." Powder Technol. 11:107-119.

Schubert, H. 1977. "Tensile Strength and Capillary Pressure of Moist Agglomerates." Proceedings of the Second International Symposium on Agglomerates; pp. 144-155. AIME Tech. Pub., New York, New York.

Schubert, H., Herrman, R., and H. Rumpf. 1975. "Deformation Behavior of Agglomerates Under Tensile Stress." Powder Technol. 11:58-62.

Selby, C.L. 1981. Rheological Properties of Kaolin and Chemically Simulated Waste. DP-1589, Savannah River Laboratory, Aiken, South Carolina. December 1981.

Stewart, C.W. 1986. Coalescence of Ellipsoidal Bubbles Rising Freely in Low-Viscosity Liquids. Ph.D. Thesis, Washington State Univ., Pullman, Washington.

Stewart, C.W., L.A. Schienbein, J.D. Hudson, E.J. Eschbach, and D.L. Lessor. 1994. Assessment of Alternative Mitigation Concepts for Hanford Flammable Gas Tanks. PNL-10105, Pacific Northwest Laboratory, Richland, Washington. September 1994.

Terrones; G., and L.L. Eyler. 1993. Computer Modeling of ORNL Storage Tank Sludge Mobilization and Mixing. PNL-8855, Pacific Northwest Laboratory, Richland, Washington. 
Tingey, J.M., P.R. Bredt, and E.H. Shade. 1994. The Effects of Heating and Dilution on the Rheological and Physical Properties of Tank 241-SY-101 Waste. PNL-10198, Pacific Northwest Laboratory, Richland, Washington. May 1994.

Trent, D.S., and L.L. Eyler. 1993. TEMPEST: A Computer Program for Three-Dimensional TimeDependent Computational Fluid Dynamics. Volume 1: Theory. Version T. Mod 2. PNL-8857, Rev. 0, Pacific Northwest Laboratories, Richland, Washington.

Tsotsas, E., and H. Martin. 1987. "Thermal Conductivity of Packed Beds: A Review." Chem. Eng. Process 22:19-37.

Turian, R.M., F.-L. Hsu, K.S. Avramidis, D.-J. Sung, and R.K. Allendorfer. 1992. "Settling and Rheology of Suspensions of Narrow-Sized Coal Particles." AIChE J. 38(7):969-987.

Wallis, G.B. 1974. "The Terminal Speed of Single Drops or Bubbles in an Infinite Medium." Int. J. Multiphase Flow 1:491-511.

Zaltash, A., and M.R. Ally. 1992. "Predicting Viscosities of Aqueous Salt Mixtures." Presented at the 1992 ASME Winter Meeting, Anaheim, California, November 7-13, 1992. CONF-921110--30, DE93 002421.

\subsection{Resources}

Adachi, K., and N. Yoshioka. 1973. "On creeping flow of a visco-plastic fluid past a circular cylinder." Chem. Eng. Sci. 28:215-226.

Agarwala, M.K., B.R. Patterson, and P.E. Clark. 1992. "Rheological behavior of powder injection molding model slurries." J. Rheol. 36(2):319-334.

Allison, J.M. 1985. Computer Prediction of Evaporator Operation. SD-WM-ADP-004, Rockwell Hanford Operations, Richland, Washington. July 1985.

Ansley, R.W., and T.N. Smith. 1967. "Motion of Spherical Particles in a Bingham Plastic." AIChE J. 13(6):1193-1196.

Astarita, G. and G. Apuzzo. 1965. "Motion of Gas Bubbles in Non-Newtonian Fluids." AIChE J. 11(5):815-820. 
Barney, G.S. 1976. Vapor-Liquid-Solid Phase Equilibria of Radioactive Sodium Salt Wastes at Hanford, ARH-ST-133, Atlantic Richfield Hanford Company, Richland, Washington. January 1976.

Beris, A.N., J.A. Tsamopoulos, R.C. Armstrong, and R.A. Brown. 1985. "Creeping motion of a sphere through a Bingham plastic." J. Fluid Mech. 158:219-244.

Bly, M.J., and R.M. Worden. 1992. "The Effects of Solids Density and Void Fraction on the Bubble Rise Velocity in a Liquid-Solid Fluidized Bed." Chem. Eng. Sci. 47(13/14):3281-3288.

Calderbank, P.H., D.S.L. Johnson, and J. Loudon. 1970. "Mechanics and mass transfer of single bubbles in free rise through some Newtonian and non-Newtonian liquids." Chem. Eng. Sci. 25:235256.

Chang, S.C., and T.R. Beaver. 1993. Development Testing of Mitigation of Tank 101-SY by Mixing. WHC-SD-WM-ER-179, Rev. 0, Westinghouse Hanford Company, Richland, Washington.

DeKee, D., P.J. Carreau, and J. Mordarski. 1986. "Bubble Velocity and Coalescence in Viscoelastic Fluids." Chem. Eng. Sci. 41(9):2273-2283.

DeKee, D., R.P. Chhabra, and A. Dajan. 1990. "Motion and Coalescence of Gas Bubbles in NonNewtonian Polymer Solutions." J. Non-Newtonian Fluid. Mech. 37:1-18.

Drew, D., and R.T. Lahey, Jr. 1987. "The Virtual Mass and Lift Force on a Sphere in Rotating and Straining Inviscid Flow." Int. J. Multiphase Flow 13(1):113-121.

Drew, D., and R.T. Lahey, Jr. 1990. "Some Supplemental Analysis Concerning the Virtual Mass and Lift Force on a Sphere in a Rotating and Straining Inviscid Flow." Int. J. Multiphase Flow 16(6):1127-1130.

Enea, O., P.P. Singh, E.M. Woolley, K.G. McCurdy, and L.G. Hepler. 1977. "Heat capacities of aqueous nitric acid, sodium nitrate, and potassium nitrate at $298.15 \mathrm{~K} ; \Delta \mathrm{C}_{\mathrm{p}}$ of ionization of water." J. Chem. Thermodyn. 9:731-734.

Gal-Or, B., and S. Waslo. 1968. "Hydrodynamics of an ensemble of drops (or bubbles) in the presence or absence of surfactants." Chem. Eng. Sci. 23:1431-1446. 
Glatzmaier, G.C., and W.F. Ramirez. 1988. "Use of Volume Averaging for the Modeling of Thermal Properties of Porous Materials.” Chem. Eng. Sci. 43(12):3157-3169.

Halley, J.W., W.K. Holcomb, and K. Goetz. 1980. "Conduction in multicomponent percolation." Phys. Rev. B 21(10):4840-4849.

Hemphill, T., W. Campos, and A. Pilehvari. 1993. "Yield-power law model more accurately predicts mud rheology." Oil Gas J., Aug. 23, 1993, p. 45-50.

Hirose, T., and M. Moo-Young. 1969. "Bubble Drag and Mass Transfer in Non-Newtonian Fluids: Creeping Flow with Power-Law Fluids." Can. J. Chem. Eng. 47:265-267.

Jarzebski, A.J., and J.J. Malinowski. "Drag and Mass Transfer in Multiple Drop Slow Motion in a Power Law Fluid." Chem. Eng. Sci. 41(10):2569-2573.

Jean, R.-H., and L.-S. Fan. 1990. "Rise Velocity and Gas-Liquid Mass Transfer of a Single Large Bubble in Liquids and Liquid-Solid Fluidized Beds." Chem. Eng. Sci. 45(4):1057-1070.

Manoliadis, O., and P.L. Bishop. 1984. "Temperature Effect on Rheology of Sludges." J. Environ. Eng. 110(1):286-290.

Marrucci, G. 1965. "Rising Velocity of a Swarm of Spherical Bubbles." Ind. Eng. Chem. Fundam. $4(2): 224-225$.

Ondracek, G. 1986. "Progress in Composites: Microstructure-Thermomechanical-Property Correlations of Two-Phase and Porous Materials." KfK 4169, Kernforschungszentrum Karlsruhe, F.R.G.

Sahimi, M., B.D. Hughes, L.E. Scriven, and H.T. Davis. 1983. "Real-space renormalization and effective-medium approximation to the percolation conduction problem." Phys. Rev. B 28(1):307311.

Sahimi, M., L.E. Scriven, and H.T. Davis. 1984. "On the improvement of the effective-medium approximation to the percolation conductivity problem." J. Phys. C 17:1941-1948. 
Sangani, A.S., D.Z. Zhang, and A. Prosperetti. 1991. "The added mass, Basset, and viscous drag coefficients in nondilute bubbly liquids undergoing small-amplitude oscillatory motion." Phys. Fluids A 3(12):2955-2970.

Shah, N., and J.M. Ottino. 1986. "Effective Transport Properties of Disordered Multi-Phase Composites: Application of Real-Space Renormalization Group Theory." Chem. Eng. Sci. 41(2):283-296.

Shahi, K., and J.B. Wagner, Jr. 1982. "Enhanced Ionic Conduction in Dispersed Solid Electrolyte Systems (DSES) and/or Multiphase Systems: $\mathrm{AgI}-\mathrm{Al}_{2} \mathrm{O}_{3}, \mathrm{AgI}-\mathrm{SiO}_{2}, \mathrm{AgI}-\mathrm{Fly}$ ash, and AgI-AgBr." J. Solid State Chem. 42:107-119.

Shima, A., and T. Tsujino. 1978. "The Behavior of Gas Bubbles in the Casson Fluid." J. Appl. Mech. 45:37-42.

Stewart, C.W., and C.T. Crowe. 1993. "Bubble Dispersion in Free Shear Flows." Int. J. Multiphase Flow 19(3):501-507.

Tran, Q.-K., D.T. Trinh, R.R. Horsley, and J.J: Relzes. 1993. "Drag Coefficients and Settling Velocities of Spheres in Yield-Pseudoplastic Slurries." AMD-Vol. 175, Developments in NonNewtonian Flows, ASME 1993, p. 131-137.

Tsai, S.C., D. Botts, and J. Plouff. 1992. "Effects of particle properties on the rheology of concentrated noncolloidal suspensions." J. Rheol. 36(7):1291-1305.

Tsutsumi, A., J.-Y. Nieh, and L.-S. Fan. 1991. "Particle wettability effects on bubble wake dynamics in gas-liquid-solid fluidization." Chem. Eng. Sci. 46(9):2381-2384.

Watson, W.G., W.C. Hahn, Jr., and R.W. Kraft. 1975. "Quantitative Microstructural Characterization of Aligned Eutectics by Electrical Analog Modeling." Metall. Trans. A 6A:151-158.

Weaire, D., N. Pittet, S. Hutzler, and D. Pardal. 1993. "Steady-State Drainage of an Aqueous Foam." Phys. Rev. Letters 71(16):2670-2673.

Zhang, D.Z., and A. Prosperetti. 1993. "Ensemble Averaged Euler Equations and Added Mass for Linearized Flows." FED-Vol. 165, Gas-Liquid Flows, ASME 1993, p. 65-70. 


\section{DISTRIBUTION}

No. of

Copies

OFFSITE

B. S. Hudson

P.O. Box 271

Lindsborg, KA 67456

K Pasamehmetoglu

Los Alamos National Laboratory

P.O. Box 1663

Mailstop K555

Los Alamos, NM 87545

\section{ONSITE}

3 DOE Richland Operations Office

J. M. Gray

M. F. Jarvis

G. Rosenwald

12 Westinghouse Hanford Company

H. Babad

W. B. Barton

D. L. Herting

J. D. Hopkins
No. of

Copies

G. D. Johnson (3)

S7-15

J. W. Lentsch

S7-12

E. J. Lipke

S7-14

N. G. McDuffie

S7-15

D. A. Reynolds

R2-11

R. J. Van Vleet

H4-62

\section{Pacific Northwest Laboratory}

Z. I. Antoniak

K7-15

S. Q. Bennett

K7-90

J. W. Brothers (5)

K5-22

J. A. Fort

K7-15

P. A. Gauglitz

P7-41

J. D. Hudson

K7-15

T. E. Michener

K7-15

F. E. Panisko

P8-34

K. P. Recknagle

K7-15

H. C. Reid

K7-15

F. M. Ryan

K7-70

A. Shekarriz

K7-15

C. W. Stewart

K7-15

D. S. Trent (5)

K7-15
S7-15

R2-11

T6-09

R2-11 